Prepared for the U.S. Department of Energy

under Contract DE-AC05-76RL01830

\title{
Final Technical Report for the Neutron Detection without Helium-3 Project
}
JH Ely
SM Robinson
M Bliss
ER Siciliano
RT Kouzes
MT Swinhoe
AT Lintereur
ML Woodring

November 2013 


\title{
DISCLAIMER
}

This report was prepared as an account of work sponsored by an agency of the United States Government. Neither the United States Government nor any agency thereof, nor Battelle Memorial Institute, nor any of their employees, makes any warranty, express or implied, or assumes any legal liability or responsibility for the accuracy, completeness, or usefulness of any information, apparatus, product, or process disclosed, or represents that its use would not infringe privately owned rights. Reference herein to any specific commercial product, process, or service by trade name, trademark, manufacturer, or otherwise does not necessarily constitute or imply its endorsement, recommendation, or favoring by the United States Government or any agency thereof, or Battelle Memorial Institute. The views and opinions of authors expressed herein do not necessarily state or reflect those of the United States Government or any agency thereof.

\author{
PACIFIC NORTHWEST NATIONAL LABORATORY \\ operated by \\ BATTELLE \\ for the \\ UNITED STATES DEPARTMENT OF ENERGY \\ under Contract DE-AC05-76RL01830 \\ Printed in the United States of America \\ Available to DOE and DOE contractors from the \\ Office of Scientific and Technical Information, \\ P.O. Box 62, Oak Ridge, TN 37831-0062; \\ ph: (865) 576-8401 \\ fax: (865) 576-5728 \\ email: reports@adonis.osti.gov \\ Available to the public from the National Technical Information Service, \\ U.S. Department of Commerce, 5285 Port Royal Rd., Springfield, VA 22161 \\ ph: (800) 553-6847 \\ fax: (703) 605-6900 \\ email: orders@ntis.fedworld.gov \\ online ordering: http://www.ntis.gov/ordering.htm
}




\section{Final Technical Report for the Neutron Detection without Helium-3 Project}

JH Ely, M Bliss, RT Kouzes, AT Lintereur, SM Robinson, ER Siciliano, MT Swinhoe, ML Woodring

November, 2013

Prepared for

the U.S. Department of Energy

under Contract DE-AC05-76RL01830

Pacific Northwest National Laboratory

Richland, Washington 99352 
PNNL-23011

\section{Executive Summary}

This report details the results of the research and development work accomplished for the 'Neutron Detection without Helium-3' project conducted during the 2011-2013 fiscal years. The primary focus of the project was to investigate commercially available technologies that might be used in safeguards applications in the relatively near term. Other technologies that are being developed may be more applicable in the future, but were outside the scope of this study.

The primary ${ }^{3} \mathrm{He}$ alternatives application investigated was for use in neutron multiplicity counters. The high capability ${ }^{3} \mathrm{He}$ multiplicity counter, the Epithermal Neutron Multiplicity Counter (ENMC), was used as the baseline requirement for alternative technologies. If a viable alternative for the ENMC is identified, it should also satisfy systems with less stringent requirements, including coincidence counters. The capability of the ENMC was chosen as the baseline requirement for the alternatives partially due to the ENMC being one of the most challenging capabilities to match, and also due to ongoing research efforts being conducted for alternatives for use in coincidence counters.

The project was a collaborative effort between Pacific Northwest National Laboratory (PNNL) and Los Alamos National Laboratory (LANL). The scope of the project was to research three commercially-available alternative technologies, leveraging the PNNL experience in researching ${ }^{3} \mathrm{He}$ alternatives for portal monitors along with the extensive knowledge of LANL in multiplicity counter design and capability. The first half of the three-year project focused on modeling and simulation to optimize models of the ENMC with alternative technologies. The second half of the project was to develop a demonstration unit using the most promising alternative to demonstrate the technology in this type of application, validate the model and simulation results, and to discover any additional areas of research needed before this type of system could be engineered and built. The three technologies investigated were boron trifluoride $\left(\mathrm{BF}_{3}\right)$ proportional tubes, boron-lined proportional tubes, and lithium fluoride mixed with zinc sulfide scintillator (LiF/ZnS).

A baseline model of the ENMC in MCNPX (developed and validated by LANL) was used as a starting point. The model was modified to support optimization investigations, and then used as a template to insert the various alternatives. Many parameters, such as the inner and outer liners, source insertion volume, and active height were maintained to minimize configuration changes. The main change that occurred was in the diameter or thickness of the detector material and moderator, which was optimized for each alternative technology. The main metrics used in the optimization of the modeling and simulation study were the efficiency and the dieaway time. The efficiency of the system needs to be large ( $66 \%$ for the ENMC) while minimizing the residence time of a neutron in the system before capture (die-away time). The efficiency is directly related to the signal collected, while the die-away time governs the necessary gate width to measure multiplicities, which in turn is proportional to the accidental coincidences, or noise, of the system.

The modeling and simulation results indicate that it would be challenging, if not impossible, to meet the ENMC capability with $\mathrm{BF}_{3}$ or boron-lined proportional tubes. The $\mathrm{BF}_{3}$ tubes are limited by the operational pressure of the gas, and have significantly less capability than the ENMC in a reasonable bounding model that increased the system footprint by $\sim 20 \%$. The boron-lined tubes are limited by the thickness of the boron lining, which needs to remain thin (order of microns) to allow the reaction products to exit the lining and be counted. Here again a reasonably sized system limit using 4725 tubes of $0.4 \mathrm{~cm}$ in diameter (straw tubes) had 
significantly less capability than the ENMC. However, an optimal configuration of the LiF/ZnS alternative was developed that could provide similar capability to the ${ }^{3} \mathrm{He}$ based ENMC.

With the results of the modeling and simulation indicating that the LiF/ZnS could outperform the other alternatives by a wide margin, and had the potential to meet the ENMC capability, a demonstrator system design was initiated and an experimental small-scale detector was developed to perform measurements to optimize the design for light collection and gamma-ray discrimination. These experiments included different types and configurations of light guides and different configurations of photomultiplier tubes (PMTs).

There are several challenges with the LiF/ZnS material for system development, and in particular for multiplicity counters. First, the modeling and simulation is not easy to perform for the whole neutron detection process, from neutron reaction to electronic signal generation, due to the material being a mixture of powders. The model in this study assumed a homogenous mixture of materials instead of a mixture of LiF and $\mathrm{ZnS}$ powders, and the simulation ended at the neutron capture in the lithium. A correction for the loss of signal from the neutron capture to the electronic signal generation at the PMT was accounted for using a Validation Correction Factor (VCF). The VCF is a parameter that is determined by comparing experimental and simulation results, and initially was calculated using a commercial LiF/ZnS paddle manufactured by Innovative American Technologies (IAT). With the building of the demonstrator system, the VCF was measured using detectors more consistent with the full-scale design, leading to a more accurate estimate of the full-scale system capability.

Another challenge of the LiF/ZnS material is that the silver-activated zinc sulfide ( $\mathrm{ZnS}: \mathrm{Ag})$ is sensitive to gamma-rays, and produces scintillation light. However, the electronic pulses arising from gamma-ray energy deposition in ZnS:Ag have a shorter decay time than from (heavy) charged particles, and therefore can be discriminated using pulse-shape discrimination. This gamma-ray discrimination adds an additional analysis step as compared to ${ }^{3} \mathrm{He}$ or boron based tubes, where the gamma-ray discrimination can be accomplished with a simple threshold on the pulse height. In addition, the ZnS:Ag has a long luminescence lifetime, on the order of several microseconds, which will result in new pulses arriving before the old pulse has decayed away (creating pulse pile-up) when the interaction rate is high. There are approaches that can be used to condition and process the signals appropriately, but this also adds another processing step that is not required for other solutions.

Research was performed to investigate approaches to separate pulses from gamma rays and neutrons and piled-up pulses from one another. This research used computer algorithms to post-process digitized data. Initially a wavelet approach was used, but a template-matching algorithm using exemplar pulses with log likelihood fitting routines was more optimal. With this approach, using synthetic data at various pile-up times, the algorithm appears to be able to differentiate gamma rays from neutrons with a high probability up to rates near the $\mathrm{MHz}$ range (average time between events on the order of $1 \mu \mathrm{s}$ ). This analysis rate will cover a wide range of assay material weights, but may be insufficient for high mass samples.

In order to understand the effect of misidentification of the gamma rays as neutrons, an investigation was performed to include gamma-ray multiplicity distributions into the multiplicity and mass equations. This was an analytical study, using published gamma-ray and neutron multiplicity distributions of plutonium, and incorporating them both into the multiplicity equations. Traditionally, the gamma-ray distributions have been neglected, as the gamma-ray discrimination is very good in ${ }^{3} \mathrm{He}$ based systems. Adding in the gamma-ray distributions complicated the equations, but for low gamma-ray detection efficiencies does not affect the overall mass estimate significantly. Using reasonable estimates for the gamma-ray 
discrimination and efficiency for a LiF/ZnS based system, the error in the mass estimate resulting from gamma-ray misidentification is on the order of $0.01 \%$, which would be a relatively insignificant error for a target uncertainty of $1 \%$, typical of these types of measurements. This study was an analytical estimate, and actual measurements would need to be performed to validate these results, but do indicate that even with a modest gamma-ray discrimination ${ }^{1}$ capability (order of $10^{-5}$ ) the LiF/ZnS material could provide a viable replacement for the ENMC for most, if not all, of the measurement scenarios.

Based on the initial bench-top experiments and data collection, a demonstrator system was developed to allow measurements in a configuration more consistent to multiplicity counting. The demonstrator consisted of four paddles that could be configured in a square around the source, to provide more uniform and complete coverage and allow for a better estimate of the efficiency and die-away time. This design represents approximately one-sixth of a full-scale system. The demonstrator also allowed exploration of issues that will be challenges for a fullscale system. The efficiency results were compared to modeling and simulation results of the demonstration system to provide a better estimate of the Validation Correction Factor. This allows for a better estimate of the performance of the full-scale system based on simulation results.

The development and assembly of the demonstrator paddles illuminated a challenge for the fullscale development in the overall height of the system. The demonstrator paddle housings are 153.7-cm (60.5-in) high, which is too high for operational use of a full-scale system. The overall height should be no higher than around $100 \mathrm{~cm}$ ( $\sim 39 \mathrm{in})$, and therefore the design for a fullscale LiF/ZnS system will need to have some unique approaches to light collection and photomultiplication in order to reduce the overall height.

The data acquisition system, which initially was a digitizer capable of collecting waveforms, was also a challenge for the demonstrator system and will be even more significant for a full-scale system. The dead time associated with capturing a waveform for subsequent processing distorted the multiplicity counting significantly, especially for the die-away time measurements. An analog set of electronics was then assembled and used to process the coincidence between the PMTs, and produce a signal, which was fed into a shift register for measuring the multiplicity distributions. This approach has the advantage of collecting the pulses without much dead time, and provides a reasonable die-away time estimate. Gamma-ray responses were minimized by requiring coincidence between the photomultiplier tubes, and also using a relatively high threshold; however, no additional pulse shape discrimination was used in this data acquisition scenario. The effect of gamma-ray responses was measured by using an unshielded and shielded (with lead) neutron source, and appears to have negligible effect.

The efficiency was measured with the analog and digital electronics for both the unshielded and lead-shielded configurations. The estimates of the efficiency were relatively consistent between the various configurations. The dead time of the digital system was measured to be approximately $12 \%$ for these configurations, with the dead time of the analog system likely similar. The raw rates (not dead-time corrected) from the analog collection were $\sim 95 \%$ of the dead-time corrected rates of the digital system. This is consistent with an assumption of the dead time of the analog system on the order of $5-10 \%$, since the gains and thresholds were not identical for the different collection methods. The gamma rays that were identified by pulse

\footnotetext{
${ }^{1}$ Gamma-ray discrimination is a measure of the ability of a neutron detector not to classify a gamma ray induced signal as a neutron, and is measured as the number of gamma rays identified as neutrons divided by the number of gamma rays incident on the detector.
} 
shape discrimination (digital acquisition) accounted for only approximately $1-2 \%$ of the overall rate, since the requirement of coincidence between the PMTs significantly reduces the acceptance of gamma rays. There was not a significant difference in the gamma-ray rate when the source was shielded with lead compared to the unshielded configuration, another indicator that the majority of gamma rays are suppressed by the coincidence requirement.

The die-away time was estimated from the measurement for this demonstrator system to be approximately $14 \mu \mathrm{s}$. This was compared to simulation results of approximately $12 \mu \mathrm{s}$ using a model of the demonstration system which is fairly consistent. This measurement, along with other configurations that were also simulated and measured, provides validation support of the implemented model and added confidence in the estimated capability for the full-scale system.

The absolute efficiency of the demonstrator system was measured to be $6-7 \%$ using pulse shape discrimination to distinguish between neutrons and gamma rays. The gamma-ray discrimination (misidentification) factor when using pulse shape discrimination was estimated to be $3 \times 10^{-8}$. The absolute efficiency was calculated as the net detected neutrons divided by the total emitted neutrons from the source.

The efficiency value was used to determine the Validation Correction Factor by forming a ratio with the simulation results. The VCF is 0.84 for this demonstrator system, which is larger than the previous VCF obtained with the IAT commercial system that uses wavelength-shifting fibers instead of plastic sheets. The increase is likely due to the use of paddles for light guides for the demonstrator instead of fibers as used in the IAT system.

The measured efficiency, and therefore the VCF, is dependent on the pulse-shape analysis parameters for gamma-ray discrimination. Increasing the gamma-ray discrimination lowers the overall neutron detection efficiency and the trade-off between sufficient gamma-ray discrimination and neutron efficiency needs further research. It may be that the majority of samples with higher gamma-ray fluxes (requiring more gamma-ray discrimination) also have higher neutron fluxes, and thus increasing the discrimination and lowering the neutron efficiency may allow adequate mass estimate precision for these high rate applications as well. An algorithm that allows for variable gamma-ray discrimination and neutron detection efficiency may provide a larger dynamic range than an approach with a fixed efficiency and gamma-ray discrimination.

Based on the measurements of the demonstrator system, the full-scale model was revisited and the capability re-estimated. The estimated capability of the full-scale system as modeled is $63 \%$ efficiency with a die-away time of approximately $10 \mu \mathrm{s}$. This is compared to the ${ }^{3} \mathrm{He}$-based ENMC with a simulated efficiency for the same configuration (point source of ${ }^{252} \mathrm{Cf}$ ) of $66 \%$ with a die-away time of $23 \mu \mathrm{s}$. Based on various figures of merit that can be calculated, it is estimated that a full-scale system using LiF/ZnS can meet or exceed the capability of the ENMC depending on the design. However, there are challenges to address with this technology including gamma-ray discrimination, pulse pile-up, and stability of a full-scale system before an operational system can be realized. Development of a full-scale system design that addresses these challenges will be the initial focus of the follow-on project, with the development and characterization of the full-scale system in the out-years. 


\section{Contents}

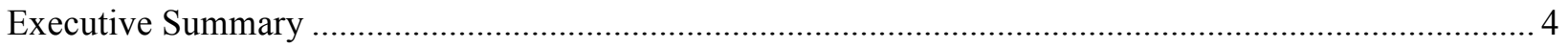

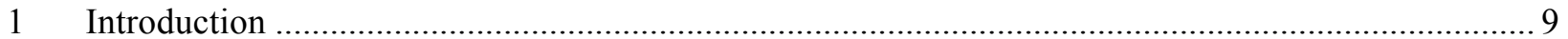

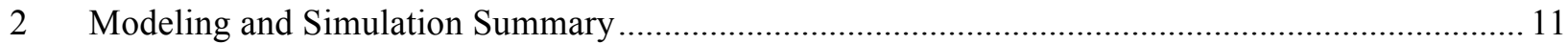

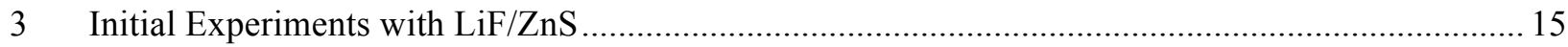

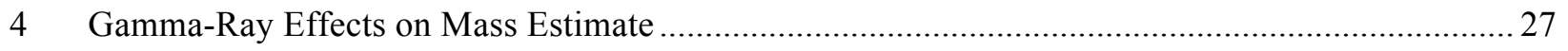

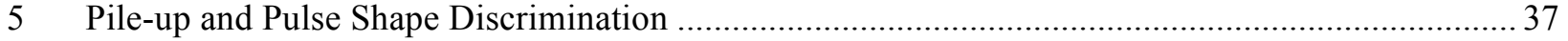

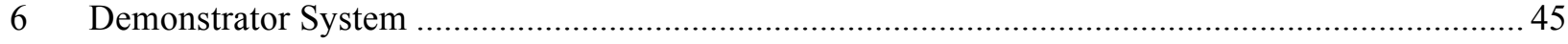

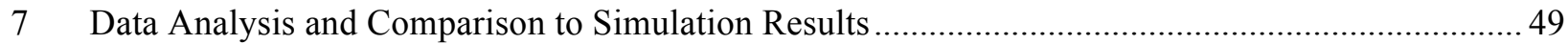

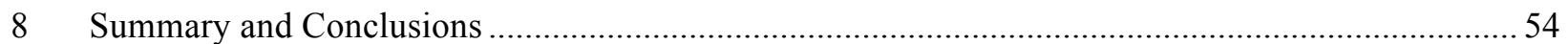

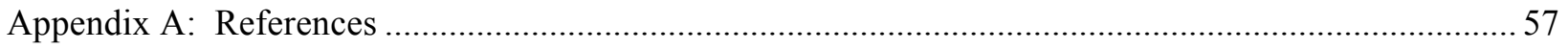


PNNL-23011

\section{Introduction}

The goal of this project was to investigate and identify alternatives to ${ }^{3} \mathrm{He}$ for neutron detection in multiplicity counters, which will be needed due to the depletion of the ${ }^{3} \mathrm{He}$ reserves and forecast of limited production [1]. The project was a collaborative effort between Pacific Northwest National Laboratory (PNNL) and Los Alamos National Laboratory (LANL). The first part of the project focused on developing models for computer simulations of alternatives to ${ }^{3} \mathrm{He}$ for multiplicity counters in safeguards applications. The last half of the project used the results of the simulation to design and build a small-scale demonstrator system using the most promising alternative technology. This demonstrator provided verification of the modeling and simulation, and provided insight into the potential challenges of building a full-scale system.

The Epithermal Neutron Multiplicity Counter (ENMC) [2] was chosen to represent the baseline capability. A model of the ENMC system (ENMC125) model, developed by LANL in the Monte Carlo N-Particle X (MCNPX) [3] simulation environment, was used as the baseline model for alternative technologies. The ${ }^{3} \mathrm{He}$ alternatives were commercially available options with boron10 and lithium- 6 materials, as they are two primary materials (other than ${ }^{3} \mathrm{He}$ ) used for thermal neutron capture. The alternative technologies include boron trifluoride proportional tubes, boron-lined proportional tubes, and lithium fluoride particles mixed with zinc sulfide particles ( $\mathrm{LiF} / \mathrm{ZnS})$ in a binder used with a form of light guides. These technologies had been identified during testing of alternatives for neutron detection modules for homeland security applications $[4,5]$. Neither boron-10 nor lithium- 6 has the same large cross-section for thermal neutron capture as ${ }^{3} \mathrm{He}$. They also have other challenges, such as detecting the reaction products for the boron-lined case, which limits their overall capability. These shortfalls pose a significant challenge to discovering an optimal configuration and approach that will provide the same performance as ${ }^{3} \mathrm{He}$ for multiplicity counter applications.

In addition to the neutron detection efficiency, another important aspect of the system is the noise or background coincidences that might be present. These background coincidences are governed by the coincidence window used by the data acquisition system to collect the neutrons that are coincident in time; minimizing the window minimizes the accidental coincidences or background of the system. The window is a function of the time to detect coincident neutrons, which for these capture materials, is the residence time of thermal neutrons in the system as the cross-section for capture is inversely proportional to the energy. The common metric to characterize the time to detect a neutron is the die-away time, which is a quantification of the time distribution of the neutron detections after the first neutron triggers the data acquisition process. In the simulation data, the doubles rates as a function of the gate width and pre-delay times are used to determine values for the die-away time $(\tau)$. Using the coincidence or doubles rates provide a more direct comparison to values resulting from actual shift register measurements, and gives more accurate die-away values, particularly for the few-microsecond region in the response of $\mathrm{LiF} / \mathrm{ZnS}$ systems. The modeling and simulation provides a method to optimize the efficiency and minimize the die-away time by varying the system configuration.

The typical approach to comparing different configurations is with a Figure-of-Merit (FoM). For this project, the figure of merit that was used in the past to optimize ${ }^{3} \mathrm{He}$ based coincidence counters was used, which provides a good metric for initial optimization [6]. The formula for this metric uses the total efficiency $\varepsilon$ (as a measure of the signal) and the die-away time $T$ (as an estimate of the background). The efficiencies are dependent on the vertical position, $Z$, and source energy distribution, $E$, but just a value measured at the detector center and with the source energy distribution is usually used. These distributions can be denoted as $\varepsilon(Z)$ and $\varepsilon(E)$, 
but for this project these dependencies are neglected and a single efficiency $\varepsilon$ is used. For the relative comparisons of the technologies and configurations investigated the primary FoM value is evaluated as simply FoM $=\varepsilon^{2} / T$. This FoM will be referred to hereafter as FoM2. For some of the studied configurations, where the results start to approach the ENMC capability, a slightly different FoM for a multiplicity counter, $\operatorname{FoM} 3=\varepsilon^{3} / T$, was also used, since a multiplicity counter counts not only singles and doubles, but also triple coincident neutrons. But the FoM3 is not quite exact as the die-away time dependency for triples is closer to $\tau^{1.5}$, and an additional FoM3a $=\varepsilon^{3} / T^{1.5}$ is used as an alternative figure of merit as well. In this report, these different figures of merit are referred to as FoM2, FoM3 or FoM3a. The figures of merit are derived quantities and are useful to compare estimated performance between configurations, but may not accurately predict system performance, as other aspects, such as signal collection, electronics, and data acquisition may not be properly accounted for. Therefore the figures of merit should be used to estimate approximate capabilities only.

Finally, there are system aspects that cannot be simulated very easily, and require development of actual configurations for measurement. Experimental measurements are also needed to validate the modeling and simulation results. The system aspects include, for example, the gamma-ray sensitivity of the system, and temperature stability. Also included are technology specific issues, such as light collection efficiency of the LiF/ZnS technology. Construction of a small-scale demonstration system using the most promising technology, the LiF/ZnS coated light guides, was carried out. The demonstrator system provided the capability to experimentally measure the efficiency and die-away times of the technology to provide additional confidence in the capability estimate of the full-scale system. The measurements also provided insight into additional challenges associated with using the LiF/ZnS material in this application, and further research topics that will need to be addressed prior to development of a field system. 


\section{Modeling and Simulation Summary}

The main modeling and simulation work that was performed on the three different technologies was previously detailed in the report 'Modeling and Simulation Optimization and Feasibility Studies for the Neutron Detection without Helium-3 Project' [7] and only a summary of the main work and results will be provided here.

Models of each of the three different alternative technologies were developed; simulations were performed and numerous optimization studies conducted. The ENMC model with ${ }^{3} \mathrm{He}$ was used as a baseline for comparison, and the models were developed and validated against this standard. For each technology, the studies started with implementing the new technology into a very similar configuration as the ENMC, and then investigating possible optimization approaches. For the $\mathrm{BF}_{3}$ alternative, the optimization involved increasing the volume of gas by using larger tubes and larger numbers of tubes. For the boron-lined tubes, the optimization was to increase the surface area of the boron lining, which was accomplished by going to smaller tubes with a significant increase in the numbers and to identify the boron-lining thickness that resulted in the largest figure of merit. The LiF/ZnS material is sufficiently different from the proportional tubes that a layered design of the LiF/ZnS material and light guides was used, and the optimization was in the thickness and numbers of the layers.

Ultimately, a bounding configuration was investigated for each technology that optimized the figures of merit while maintaining a reasonable footprint (not significantly larger than the ENMC). For the $\mathrm{BF}_{3}$ case, the reasonable bounding configuration was with 155 tubes, each $5.08 \mathrm{~cm} \mathrm{(2}$ in) in diameter and filled with two atmospheres of $\mathrm{BF}_{3}$, which had a footprint approximately $20 \%$ larger in each direction (except height). A top view schematic of the model is shown below in Figure 1. This is compared to the ${ }^{3} \mathrm{He}$ baseline of $121,2.54-\mathrm{cm}$ (1-in) diameter tubes filled to ten atmospheres.

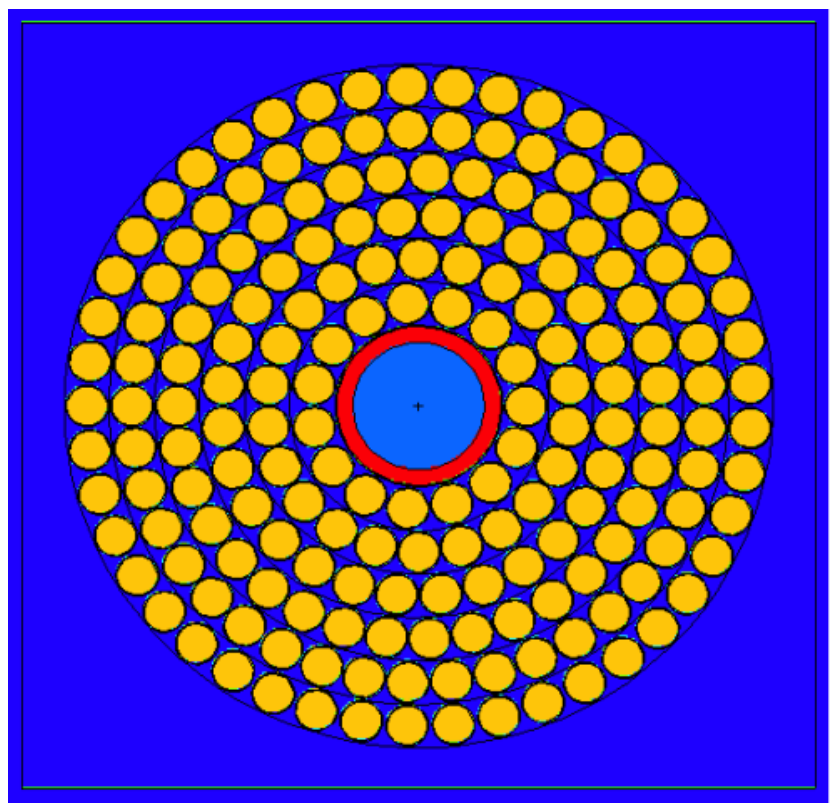

Figure 1. Bounding model for the $\mathrm{BF}_{3}$ technology with 155 tubes in six rings, each $5.08 \mathrm{~cm}$ in diameter. The system size is increased from 65.1 to $100.7 \mathrm{~cm}$ square compared to the ENMC, although the height is the same. 
For the boron lined proportional tube alternative, the bounding case consisted 4725 tubes each of $0.4 \mathrm{~cm}$ in diameter (straw tubes), with the same footprint as the ENMC, and a similar model schematic shown in Figure 2. The boron-lining thickness was optimized to one micron.
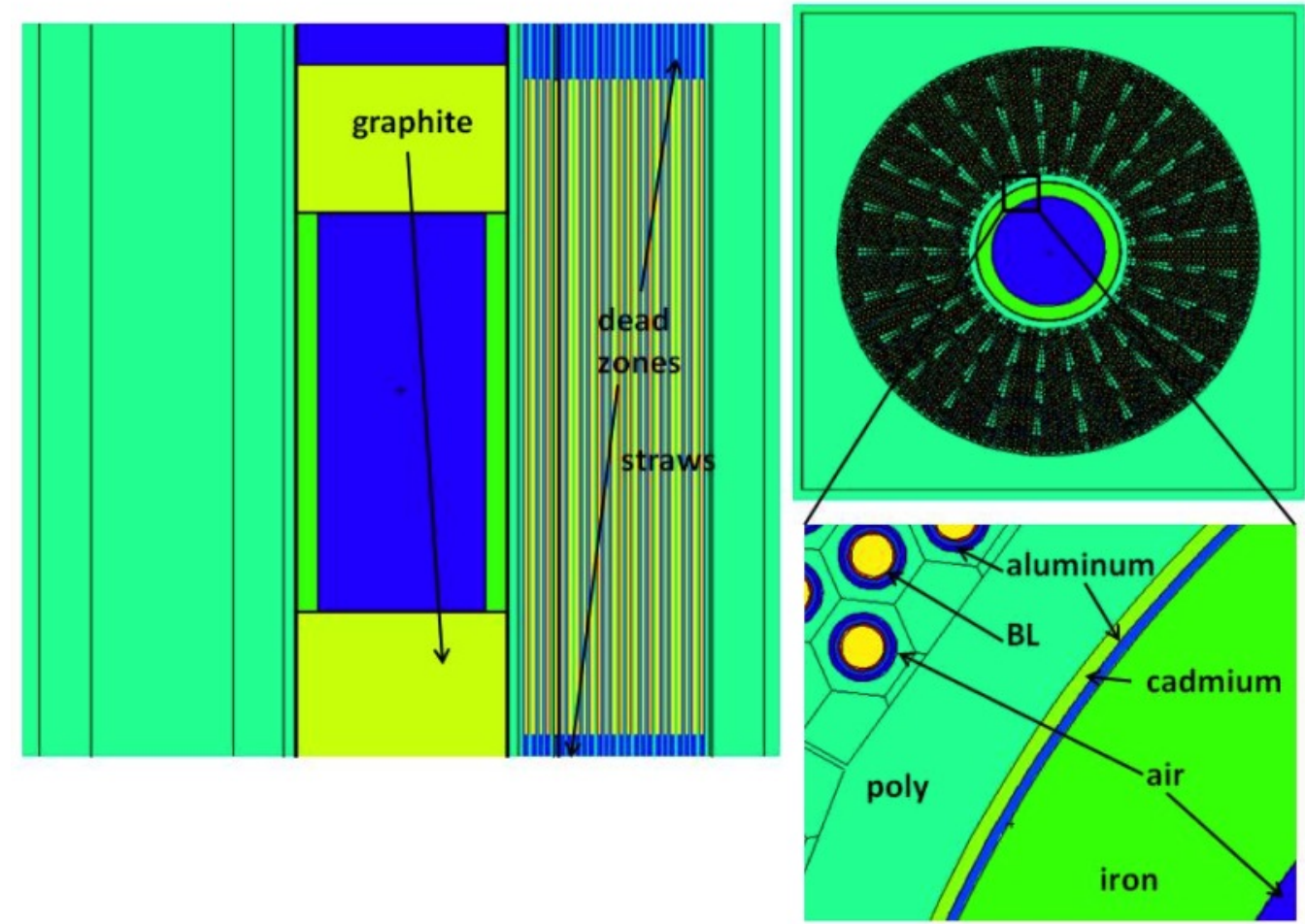

Figure 2. Bounding model of the boron-lined technology with the $4 \mathrm{~mm}$ tubes (4725 tubes).

For the boron-lined model, the reaction products were tracked and the number and energy of reaction products counted that enter into the counting gas of the proportional tube. This is a relatively new approach to modeling boron-lined tubes. The tracking is accomplished in MCNPX using a version Beta 2.7b (or newer) with the Neutron Capture lon Algorithm physics option activated. There are several approaches in MCNPX to estimate the number of neutrons detected based on the reaction products entering the counting gas. First, surface tallies can be collected on the current entering the counting gas filled area of the tube. This provides the energy and number for both the alpha and lithium ions entering the tube, and can be summed to provide the total energy deposited. Since the products are produced with opposite momentum, there is little possibility for double counting. Another approach is to use pulse height tallies that can be performed using the pulse height light anti-coincidence treatment, or a regular Type 8 tally.

The relatively new coincidence tally [8], originally designed for ${ }^{3} \mathrm{He}$ tubes, was updated to allow for use with boron-lined tubes, and is now available in MCNP6 [9]. This allows a more direct comparison of the simulation results with the experimental results produced from shift registers.

Research was performed to validate the correct simulation of the boron-lined tubes and reaction product tracking. Simulations of pulse height distributions from MCNP output, which are based on the energy deposition of the reaction products in the counting gas, were compared to experimental pulse height distributions. There is some effect of the pulse generation in the gas 
on the pulses, and to account for that process, a simulation in the electric field and gas avalanche simulation program Garfield [10] was performed, resulting in a better match of the simulation results to the experiments. Additional details on boron-lined proportional tube modeling, simulation and validation are available [11-13].

The LiF/ZnS technology was optimized to 20 alternating layers of LiF/ZnS and wavelength shifting plastic scintillator material as shown in the model in Figure 3 below. The LiF/ZnS layer thickness was $0.05 \mathrm{~cm}$ (500 microns) while the wavelength shifting plastic was optimized to $0.7-\mathrm{cm}$ thickness. For the model, a homogenous mixture of the LiF, $\mathrm{ZnS}$, and hydrogenous binder material in thin sheets was used, which does not reflect the actual distribution of the LiF and $\mathrm{ZnS}$ powders mixed with the binder. Therefore, the simulation was ended when the neutron was captured in the lithium. The MCNP (tally) method used for calculating the total count efficiencies (TCEs) in all of the LiF/ZnS neutron multiplicity counter models was based on counting the total number of $n$-captures by ${ }^{6} \mathrm{Li}$ atoms in the LiF/ZnS scintillation layers. This method ignores real-system inefficiencies that might arise from inhomogeneity in the crystal distribution and also the loss of scintillation light as it travels from the $\mathrm{ZnS}$ through the light guide to the photomultiplier tube. Accordingly, the model neutron capture rates were expected to over-estimate measured detection count rates, and a re-calibration factor was needed to estimate actual detection efficiencies. This Validation Correction Factor (VCF) is determined by comparing experimental to simulated results, for an actual system. Initially, the system used to obtain the VCF was the LiF/ZnS-based system developed by Innovative American Technologies (IAT) that was previously measured at PNNL [14]. The value of the VCF was 0.57 based on the IAT system, and the simulated efficiencies were multiplied by the VCF to predict the efficiency of the modeled system during the modeling and simulation phase of this project. Once the demonstrator system was developed, additional measurements of the VCF were carried out, which provide a VCF more consistent with the technology configuration of the full-scale system. The measurements and results will be provided in more detail in following sections of this report.

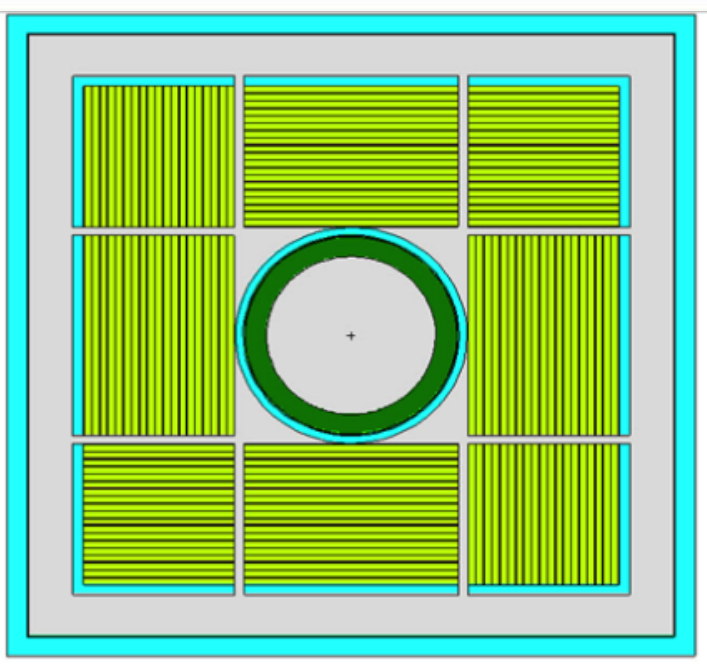

Figure 3. Bounding model of the $\mathrm{LiF} / \mathrm{ZnS}$ alternative technology with the 20 alternating layers of $\mathrm{LiF} / \mathrm{ZnS}$ material (500 microns) and wavelength shifting light guide $(0.7 \mathrm{~cm})$.

In order to summarize the data in a graphical manner, plots were developed where the efficiency is plotted on one axis and the die-away time on the other. In this representation, constant values of the FoM can be overlaid as contours. These plots show that a given target FoM value can be reached by increasing the efficiency, reducing the die-away time, or any combination thereof. The bounding models that maximized the figures of merit are provided in 
Figure 4 for the three alternative and the ENMC simulation results. Overlaid on the data are constant FoM2 (left hand plot) and FoM3 (right hand plot) values providing references for comparison. The boron-lined straw tube bounding model with 4725 tubes has fairly low efficiency and also larger die-away times compared to the ENMC. The $\mathrm{BF}_{3}$ bounding case with 99 tubes has increased efficiency compared to the boron-lined tubes, but also larger die-away times, resulting in figure of merit values 1.5-2.5 larger than the boron-lined bounding case, but still a factor of 2-3 lower than the ENMC. The LiF/ZnS bounding case of 20 layers also has lower simulated efficiency compared to the ENMC, but a shorter die away time, resulting in a FoM2 value that exceeds the ENMC, and a FoM3 that is slightly lower.
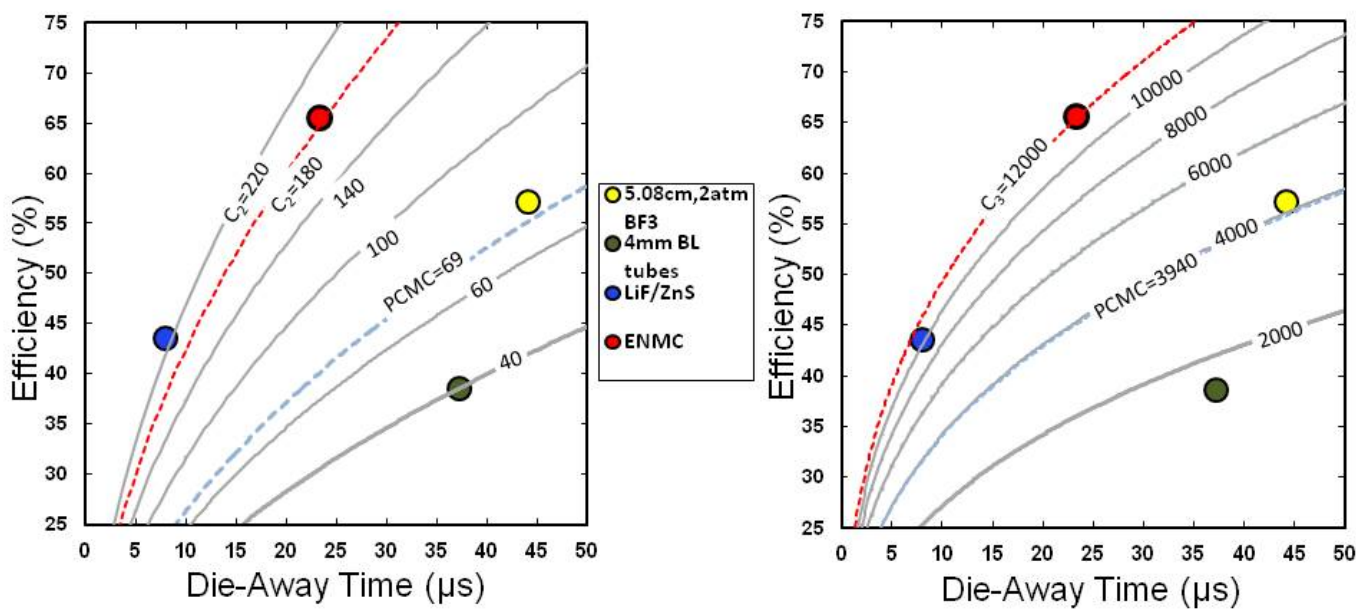

Figure 4. Summary of the optimized modeling results of the three alternatives along with the ${ }^{3} \mathrm{He}$ baseline. The constant FoM curves are overlaid on the data: FoM2 of the left and FoM3 on the right along with the ENMC values (dashed lines).

From these results it appeared that the LiF/ZnS alternative has the best possibility to meet the ENMC capability, and was selected as the alternative to move forward with for the demonstrator system.

The values for the efficiencies and die-away times from the simulations of the ENMC and alternatives for the bounding cases are provided in Table 1 for all three figures of merit. The figures of merit for the $\mathrm{BF}_{3}$ and boron-lined simulated bounding scenarios are significantly below that of the ENMC. However, the simulation results for the modeled LiF/ZnS configuration exceed the ENMC for the FoM2 and FoM3a. The FoM3 is the most conservative figure of merit favoring increased efficiency, and indicates the LiF/ZnS alternative, as modeled, may be below the ENMC capability. However, these estimated capabilities using the various figures of merit from simulation are only approximations; actual performance will need to be determined with a system.

Table 1. Simulated efficiencies and die-away times for the various alternatives. Note the $\mathrm{LiF} / \mathrm{ZnS}$ results use the initial VCF of 0.57, which is lower than the VCF obtained with the demonstrator system described later.

\begin{tabular}{|l|c|c|c|c|}
\hline & ${ }^{3} \mathbf{H e}$ & $\mathbf{B F}_{\mathbf{3}}$ & Boron-lined & LiF/ZnS \\
\hline Count Efficiency $(\%)$ & 66 & 57 & 39 & 44 \\
\hline Die-away time $(\mu \mathrm{s})$ & 23 & 44 & 37 & 10 \\
\hline FoM2 $\left(\varepsilon^{2} / \tau\right)$ & 189 & 74 & 41 & 194 \\
\hline FoM3 $\left(\varepsilon^{3} / \tau\right)$ & 12500 & 4209 & 1603 & 8518 \\
\hline FoM3a $\left(\varepsilon^{3} / \tau^{1.5}\right)$ & 2606 & 635 & 264 & 2695 \\
\hline
\end{tabular}




\section{Initial Experiments with LiF/ZnS}

In order to develop an optimized design for the demonstrator unit, and explore possibilities for the full-scale system, initial experiments were conducted with the LiF/ZnS material in a benchtop detector. These experiments also provided some initial understanding of the material and possible challenges for building and operating these types of detectors. The optimizations included using several different thicknesses of light guides, two different types of light guides, and various options for PMT configurations. The experiments included initial explorations of gamma-ray sensitivity and pulse shape discrimination capability of the ZnS material.

The LiF/ZnS technology uses lithium fluoride (LiF) powder mixed with silver activated zinc sulfide (ZnS:Ag) powder in a binder material. The lithium is enriched in lithium-6 to $96 \%$ typically, and is used as lithium-fluoride due to the highly reactive nature of pure lithium with water. The particles of both materials are on the order of 10 micrometers $(\mu \mathrm{m})$, in order to provide close contact with each other, and held together by an epoxy type of binder material. The neutrons are captured in the lithium, and emit a triton and alpha particle in the capture reaction. These charged particles interact in the surrounding material and deposit energy into the zinc sulfide scintillator material. Silver activated zinc sulfide is a bright scintillator $(\sim 160,000$ photons per neutron). However, it is a white material (opaque) due to the differences in refraction indices and the granularity of the material, and it thus attenuates the generated scintillation light quickly. Therefore, only thin sheets of LiF/ZnS are useful, on the order of 500 $\mu \mathrm{m}$ or less, and the light needs to be collected outside the sheet. There are various methods to collect the light, from large sheets of light guides to wavelength shifting materials such as optical fibers.

The initial design of the detector was chosen to be 71.12-cm (28-in) active height by 15.24-cm (6-in) wide by approximately $5.08-\mathrm{cm}$ (2-in) thick. The height was chosen to match the active height of the ENMC. The thickness was chosen as a good match for a 5.08-cm diameter (2-in) PMT, and the width a balance between increased frontal area and light collection capability for the PMT. The thickness was achieved by using five layers of the LiF/ZnS sandwiched between six layers of light guide. The overall detector thickness was dependent on the thickness of the light guides used, and varied from $3.5 \mathrm{~cm}(0.5 \mathrm{~cm}$ light guides) to $6.8 \mathrm{~cm}(0.9 \mathrm{~cm}$ light guides $)$.

Sheets of LiF/ZnS $(71.28 \mathrm{~cm} \times 15.24 \mathrm{~cm})$ were purchased from Eljen Technology, Sweetwater, TX (the sheets were a customized version of EJ-426HD2). The sheets consisted of a 500- $\mu \mathrm{m}$ thick layer comprised of a $1: 2$ ratio of ${ }^{6} \mathrm{LiF}: \mathrm{ZnS}$ particles suspended in an organic binder. The individual particles of ${ }^{6} \mathrm{LiF}$ and $\mathrm{ZnS}$ were less than $10 \mu \mathrm{m}$ in diameter (see Figure 5 below). The ${ }^{6} \mathrm{LiF} / \mathrm{ZnS}$ compound was sandwiched between two polyester sheets (each $250 \mu \mathrm{m}$ thick) by the manufacturer for support, resulting in each layer being a total of $1000 \mu \mathrm{m}$, or $1 \mathrm{~mm}$, thick. 


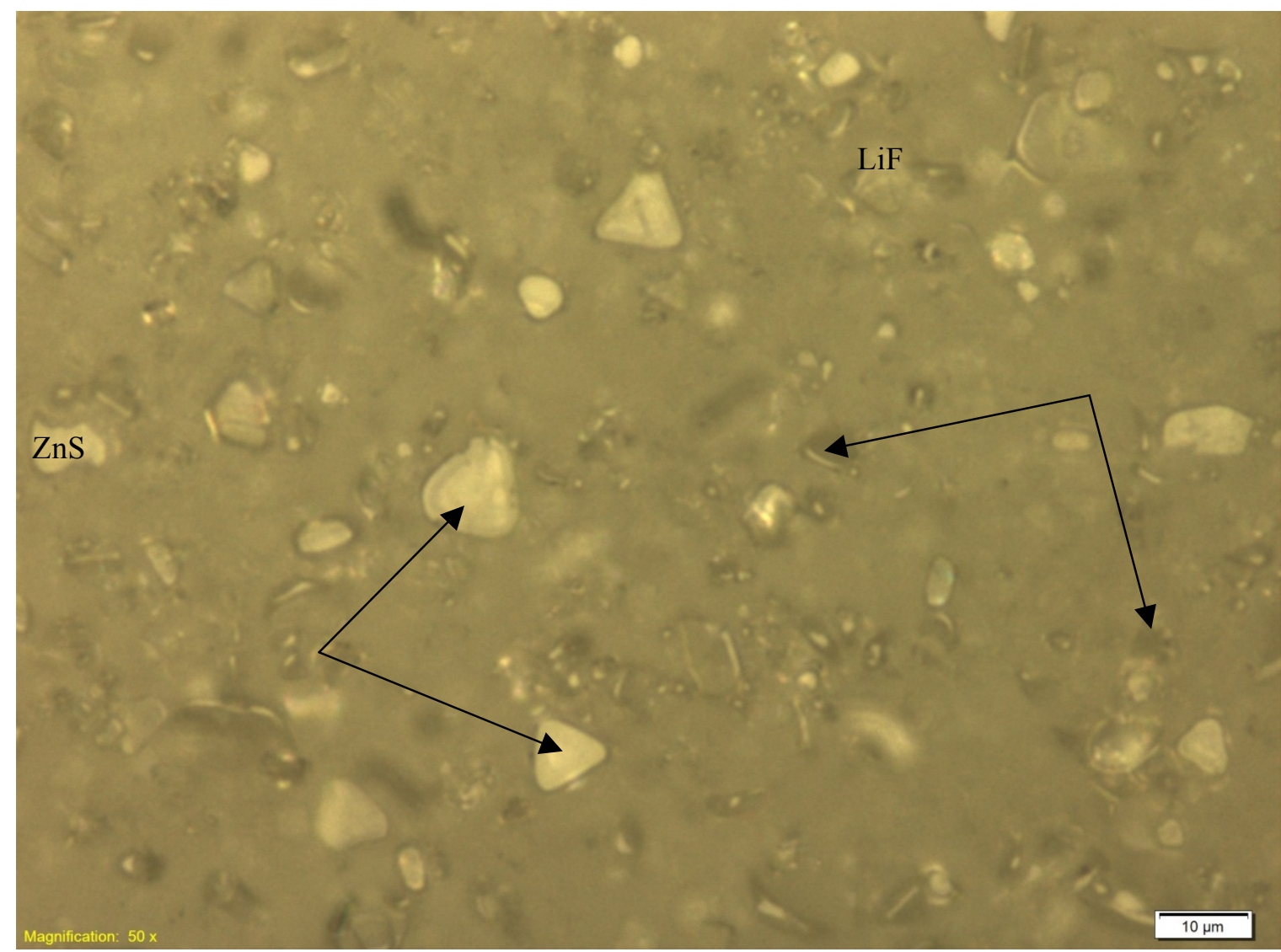

Figure 5. Magnified (50x) view of a section of a ${ }^{6} \mathrm{LiF} / \mathrm{ZnS}$ sheet (constructed by Eljen Technology) showing the individual ${ }^{6} \mathrm{LiF}$ and $\mathrm{ZnS}$ pieces suspended in the binder.

Two different light guides, poly-methyl methacrylate (PMMA) and wavelength shifting plastic (WLSP) made from polyvinyl-toluene (or PVT) (EJ-280), of 0.7-cm thickness, were purchased and tested. Photographs of each material are shown below in Figure 6. The WLSP scintillates in response to gamma rays, unlike the PMMA (although the WLSP contains a dopant that produces significant gamma-ray suppression compared to "normal" gamma-ray sensitive plastic, such as PVT). Both the WLSP and the PMMA will transmit light to the PMTs; however, the location of origin of the light that is transmitted is different between the two materials. The WLSP captures the light emitted by the ZnS and re-emits it isotropically at a different (longer) wavelength with a quantum efficiency of $95 \%$ (as reported by the manufacturer). Therefore, the light that is transmitted to the PMTs originates within the WLSP sheets as opposed to the PMMA, which does not scintillate. The WLSP plastic transmits more of the ZnS generated light to the PMT in a $0.7-\mathrm{cm}$ light guide compared to the non-scintillating PMMA, however the WLSP is somewhat gamma ray sensitive. The tradeoff between efficiency and gamma ray rejection using the WLSP or PMMA was the goal of the experimental testing. 

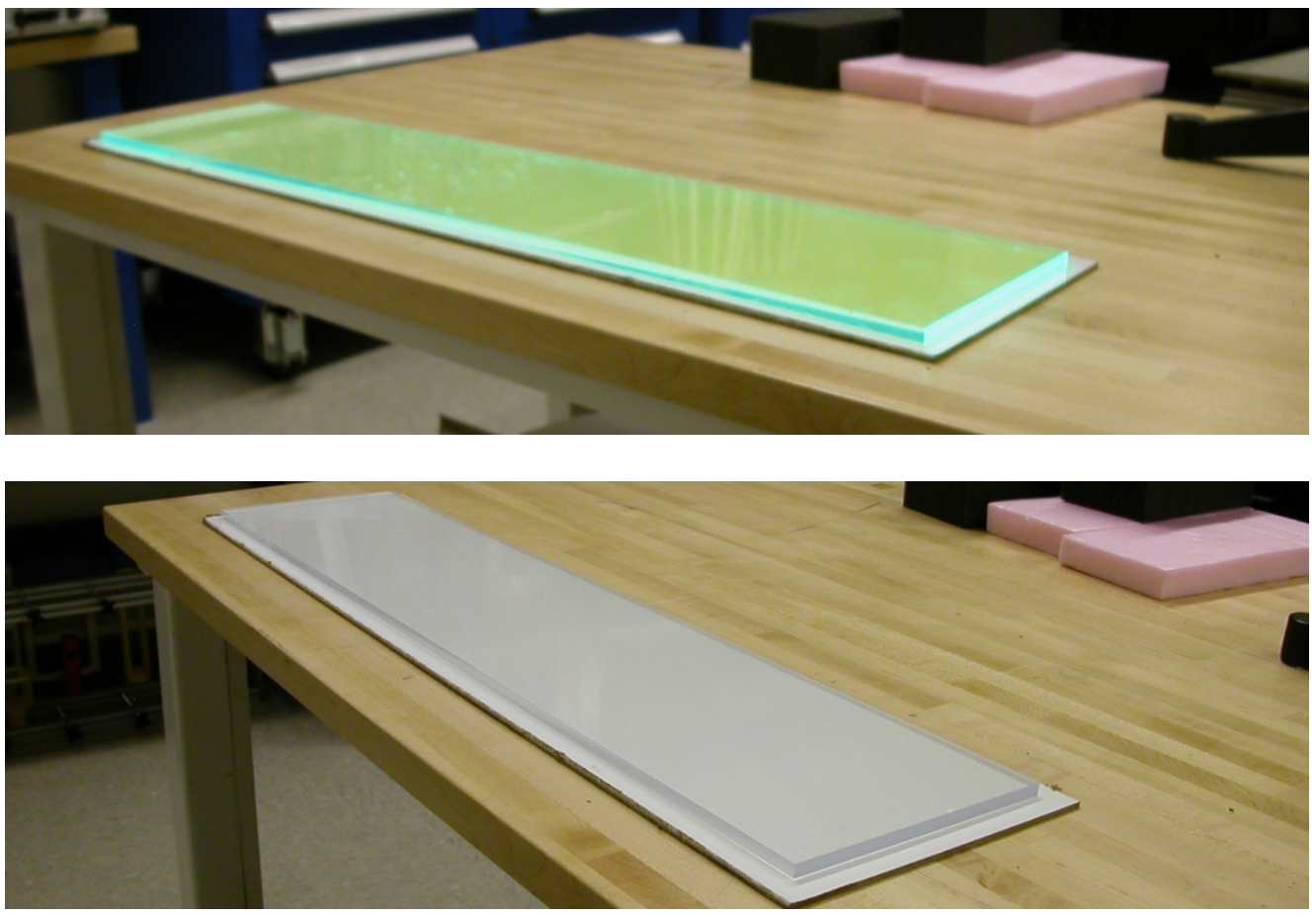

Figure 6. The WLSP (top) and PMMA (bottom) sheets used for the bench-top test system.

Three different thicknesses of the wavelength shifting plastic $(0.5 \mathrm{~cm}, 0.7 \mathrm{~cm}$, and $0.9 \mathrm{~cm})$ were used to explore the different light collection and moderation effects. The optimal thickness for neutron capture from the modeling and simulation was $0.7 \mathrm{~cm}$, with the other thicknesses tested to investigate the balance between neutron capture and light collection efficiency. The assembled bench-top experimental detector is shown below in Figure 7, where the detector has been wrapped in Teflon tape to minimize light loss.

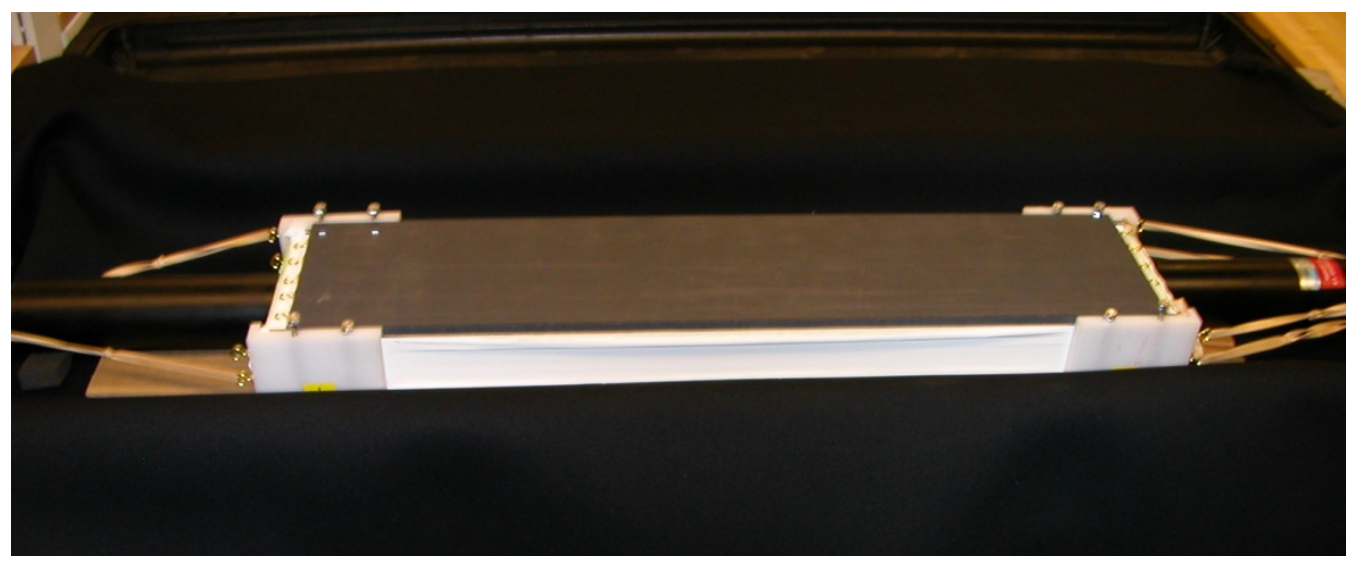

Figure 7. Bench-top test unit assembled on a support structure with two PMTs and no tapered light guides.

Measurements were made with a single PMT on one end of the detector, and with one PMT on each end of the unit. The dual PMT configuration was analyzed both for singles rates and 
coincidence (between the PMTs) rates. The measurements were performed both with and without the use of tapered light guides between the detector and the PMTs. The base of the tapered light guides matched the dimensions of the ends of the configured unit (with the $0.7-\mathrm{cm}$ thick plastic sheets) and was tapered (based on a design selected by Eljen Technology for optimal light collection efficiency) to match the 5.08-cm (2-in) diameter PMTs. The tapered light guides added length and expense to the test unit, but increased the number of photons detected by the PMTs. These competing factors were considered during the measurements. The test unit with the tapered light guides attached is shown in Figure 8. Due to the visible light sensitive nature of the detectors, the measurements were made with the system wrapped in Teflon and placed in a light-tight box. Light leaks were eliminated prior to any measurements, and the system was allowed a minimum of 24 hours settling time after an exposure to room light before measurements were performed.

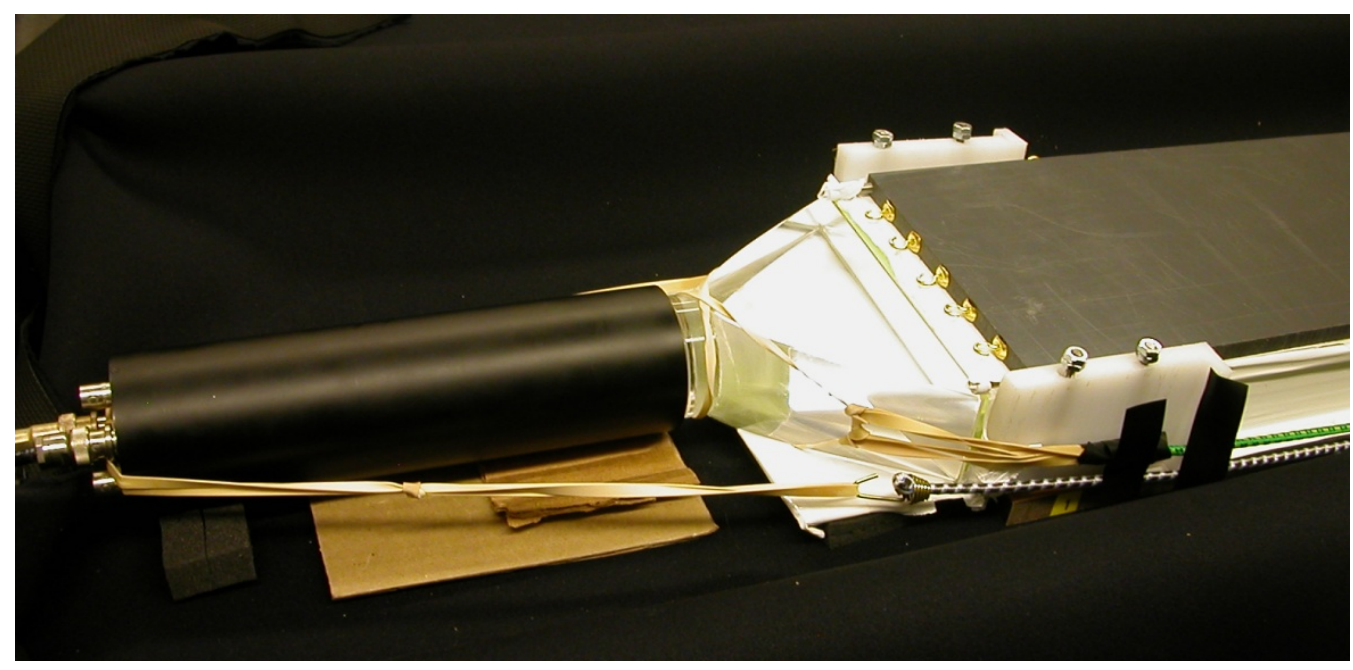

Figure 8. Test unit with a tapered light guide attached.

The photomultiplier tubes used to collect the signals were selected for a fast response and high sensitivity to blue and green wavelengths. The measurements with the test unit were made with negatively biased H1161 PMTs (manufactured by Hamamatsu). The PMTs were gain matched for the dual PMT measurements. The signals produced by the PMTs were digitized, to preserve the waveforms. The initial testing utilized a XIA (Hayward, CA) Pixie-500 for the digitization of the pulses. The trace length was set to $4 \mu \mathrm{s}$ (with a $1 \mu \mathrm{s}$ offset to establish a baseline) to collect the entire digitized neutron pulse. The neutron pulse tails extended beyond the $4 \mu \mathrm{s}$ window but the remaining signal was too low to trigger a new pulse and the additional charge wasn't necessary for the initial pulse shape discrimination (PSD) algorithm. The digitization rate of the Pixie-500 is $500 \mathrm{MHz}$, so each bin of the digitized trace was $2 \mathrm{~ns}$ in duration. All of the traces collected were stored for post-processing. A low threshold was selected for all of the measurements to maximize the recorded signal. A software threshold was set during the post processing to determine the effect of raising the threshold on both the gamma-ray and neutron detection efficiency.

The digitized traces were analyzed in a post-processing PSD to distinguish between gamma-ray responses and the signals produced in response to neutrons. For the initial bench-top configuration, the PSD was performed with a standard two-window technique. The PSD 
compared the area under the tail of the pulse to the area under the entire pulse (Figure 9). The area in the two regions was calculated by integrating the trace over specified regions of interest.

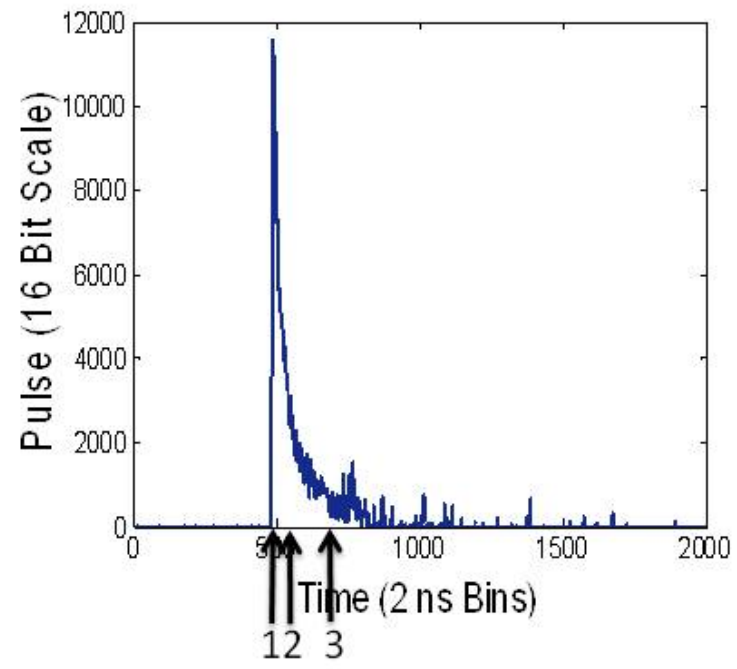

(a)

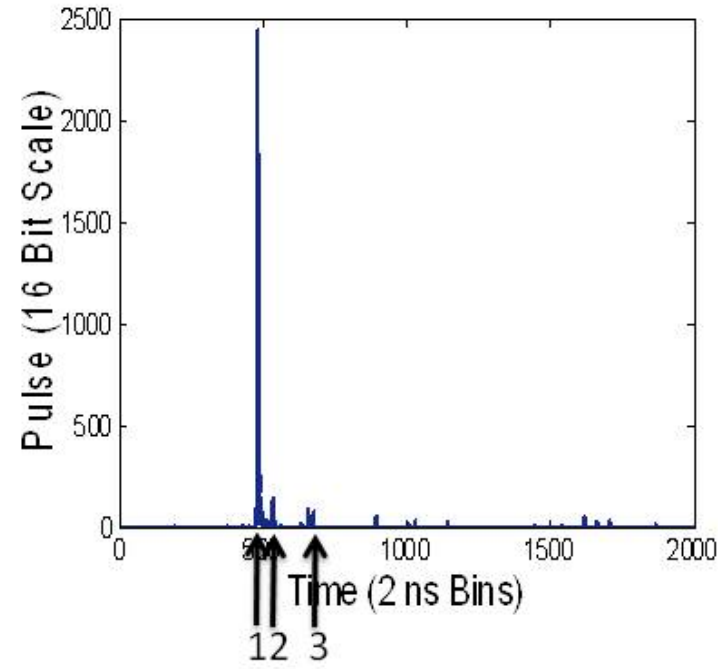

(b)

Figure 9. Neutron (a) and gamma ray (b) digitized traces illustrating the regions of charge integration for the PSD methodology applied. The entire pulse was integrated from arrow 1 to arrow 3 and the tail of the pulse from arrow 2 to arrow 3.

The integral ratios calculated from the traces were binned into histograms to determine the neutron and gamma-ray count rates, as shown in Figure 10. Note that the histogram shown in Figure 10 was generated using data that was collected with a neutron $\left({ }^{252} \mathrm{Cf}\right)$ source and a gamma-ray $\left({ }^{60} \mathrm{Co}\right)$ source measured simultaneously. The neutron source was centered above the detector and the gamma-ray source was located $10 \mathrm{~cm}$ from the PMT (or $25.56 \mathrm{~cm}$ closer to the PMT than the neutron source). The gamma-ray source was positioned closer to the PMT to obtain approximately equal neutron and gamma-ray regions in the PSD histogram. 


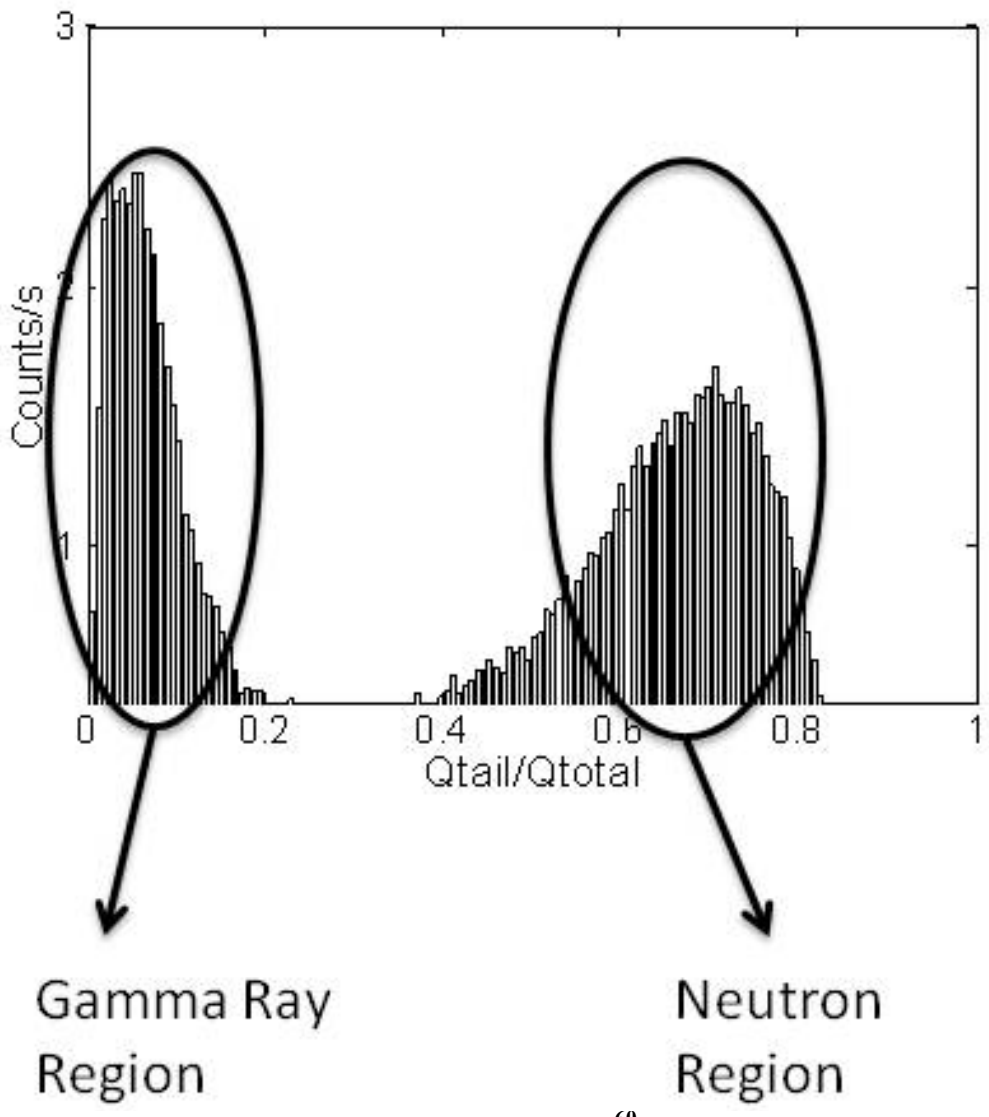

Figure 10. Histogram illustrating the charge ratio region from the ${ }^{60} \mathrm{Co}$ gamma-ray traces and the ${ }^{252} \mathrm{Cf}$ neutron traces.

The neutron detection efficiency and gamma-ray rejection capabilities were measured with the different test unit configurations. The configurations tested are summarized in Table 2 . The first sets of measurements were performed to compare the neutron detection efficiency and the gamma-ray rejection capability between the PMMA and WLSP (using the 0.7-cm thick light guides). The next set of measurements compared the neutron detection efficiency between the $0.5,0.7$, and $0.9-\mathrm{cm}$ thick WLSP sheets. Measurements were then performed with different PMT configurations, and with the tapered light guides.

Table 2. Bench-top detector configuration summary. LG refers to measurements with the tapered light guide.

\begin{tabular}{|c|c|}
\hline Light Guide & PMT Configuration \\
\hline PMMA $0.7 \mathrm{~cm}$ & 1 PMT \\
\hline WLSP $0.5 \mathrm{~cm}$ & 1 PMT \\
\hline WLSP $0.7 \mathrm{~cm}$ & 1 PMT \\
\hline WLSP $0.9 \mathrm{~cm}$ & 1 PMT \\
\hline PMMA $0.7 \mathrm{~cm}$ & 2 PMTs \\
\hline WLSP $0.5 \mathrm{~cm}$ & 2 PMTs \\
\hline WLSP $0.7 \mathrm{~cm}$ & 2 PMTs \\
\hline WLSP $0.9 \mathrm{~cm}$ & 2 PMTs \\
\hline WLSP $0.7 \mathrm{~cm}$ & 1 PMT with LG \\
\hline WLSP $0.7 \mathrm{~cm}$ & 2 PMTs with LG \\
\hline
\end{tabular}


The neutron source measurements were performed with ${ }^{252} \mathrm{Cf}$. The gamma-ray measurements were performed with ${ }^{60} \mathrm{Co}$ and ${ }^{137} \mathrm{Cs}$ sources. The top of the detector (the top piece of light guide) was $3.8 \mathrm{~cm}$ from the top of the detector holder when the $0.5-\mathrm{cm}$ thick light guides were used. The source was raised for the measurements with the thicker light guides to preserve the source to detector distance. The integrated regions for the complete trace and the tail of the trace used in the post-analysis PSD, for all the traces, were $0-600$ ns and $30-600 \mathrm{~ns}$ respectively. The length of the window for the tail pulse was selected based on the width of the gamma-ray pulses as shown in the example below (Figure 11). Note that the peak heights have been aligned for this figure, not the trigger location, which accounts for the slight offset in the pulse leading edge.

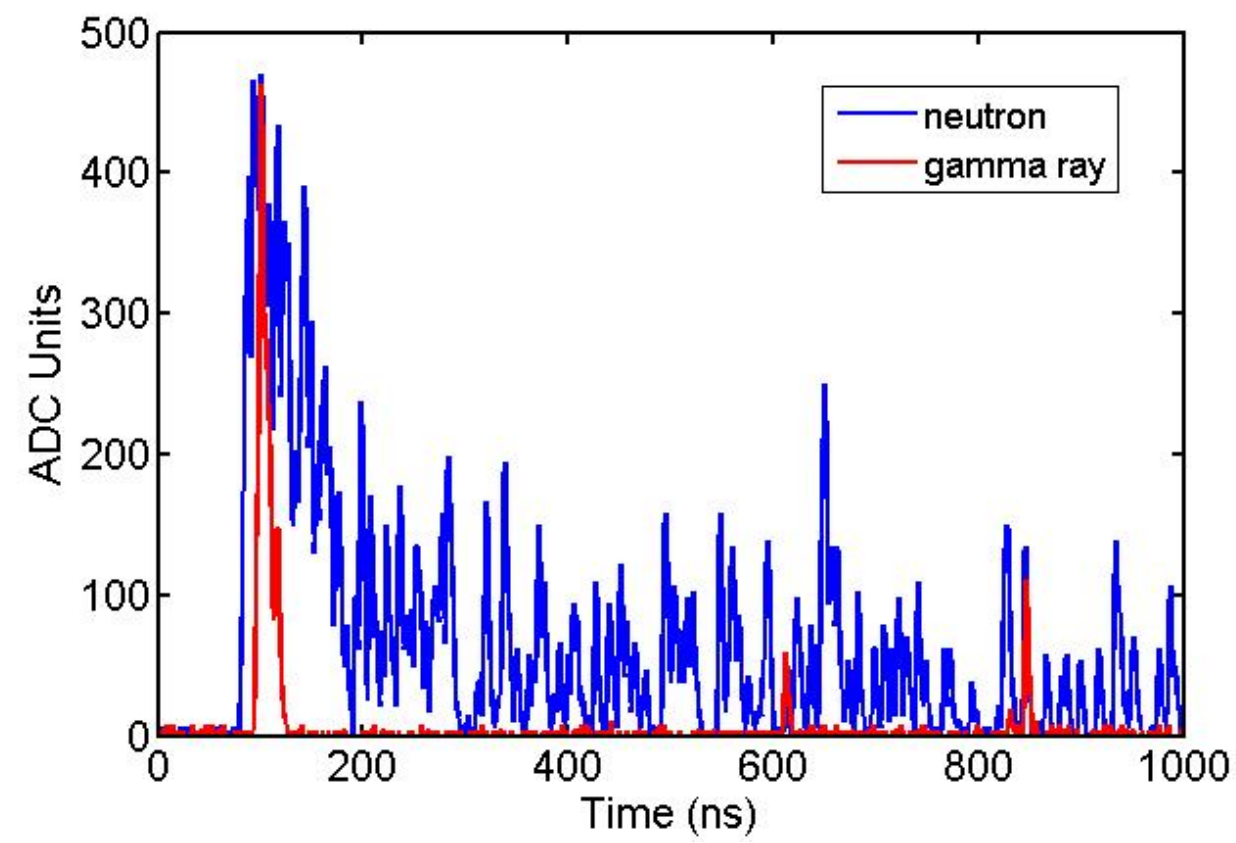

Figure 11. Example gamma-ray and neutron traces recorded with the Pixie-500 with peak heights aligned.

The neutron and gamma-ray count rates for the measurements were estimated by integrating over the gamma-ray and neutron regions for the charge ratio histograms ( 0 to 0.5 for the gamma-ray region and 0.5 to 1 for the neutron region for most of the measurements). A summary of the absolute neutron detection efficiency estimates with the $0.7-\mathrm{cm}$ thick light guides for the source position in the middle of the detector is presented in Table 3.

Table 3. Results from the ${ }^{252} \mathrm{Cf}$ measurements with the 0.7-cm thick PMMA and WLSP with a single PMT coupled directly to the end of the detector.

\begin{tabular}{|c|c|}
\hline $\begin{array}{c}\text { Measurement } \\
\text { Configuration }\end{array}$ & $\begin{array}{c}\text { Absolute Neutron } \\
\text { Detection Efficiency }\end{array}$ \\
\hline PMMA $^{252} \mathrm{Cf}$ & $0.016 \pm 0.002$ \\
\hline $\mathrm{WLSP}^{252} \mathrm{Cf}$ & $0.040 \pm 0.006$ \\
\hline
\end{tabular}


The second set of measurements was made with the same detector configurations (0.7-cm thick PMMA and WLSP) and two PMTs (one on each end, both coupled to the detector surface with a silicone rubber pad). Each PMT was connected to a separate input channel of the Pixie-500 (both set with the same trigger and energy filter settings). The Pixie-500 was configured such that output traces were recorded only if both PMTs were triggered within 13.33 ns of each other. It should be noted that for these measurements coincidence refers to a temporal coincidence between two PMTs, not a temporal coincidence between two neutrons. The coincident measurements slightly decreased the neutron detection efficiency, but resulted in a greater suppression of the gamma-ray response, as the lower intensity gamma-ray signal is less likely to be detected by both PMTs than the neutron signal. The comparison between the single PMT and the coincident PMT measurements for the WLSP detector configurations is shown in Figure 12. The PMMA configuration suffered from a greater reduction in the recorded neutron count rate when operated in coincidence mode than did the WLSP configuration (provided in

Table 4). The coincidence requirement significantly reduces the efficiency of the detector with the PMMA light guide compared to the single PMT results above.

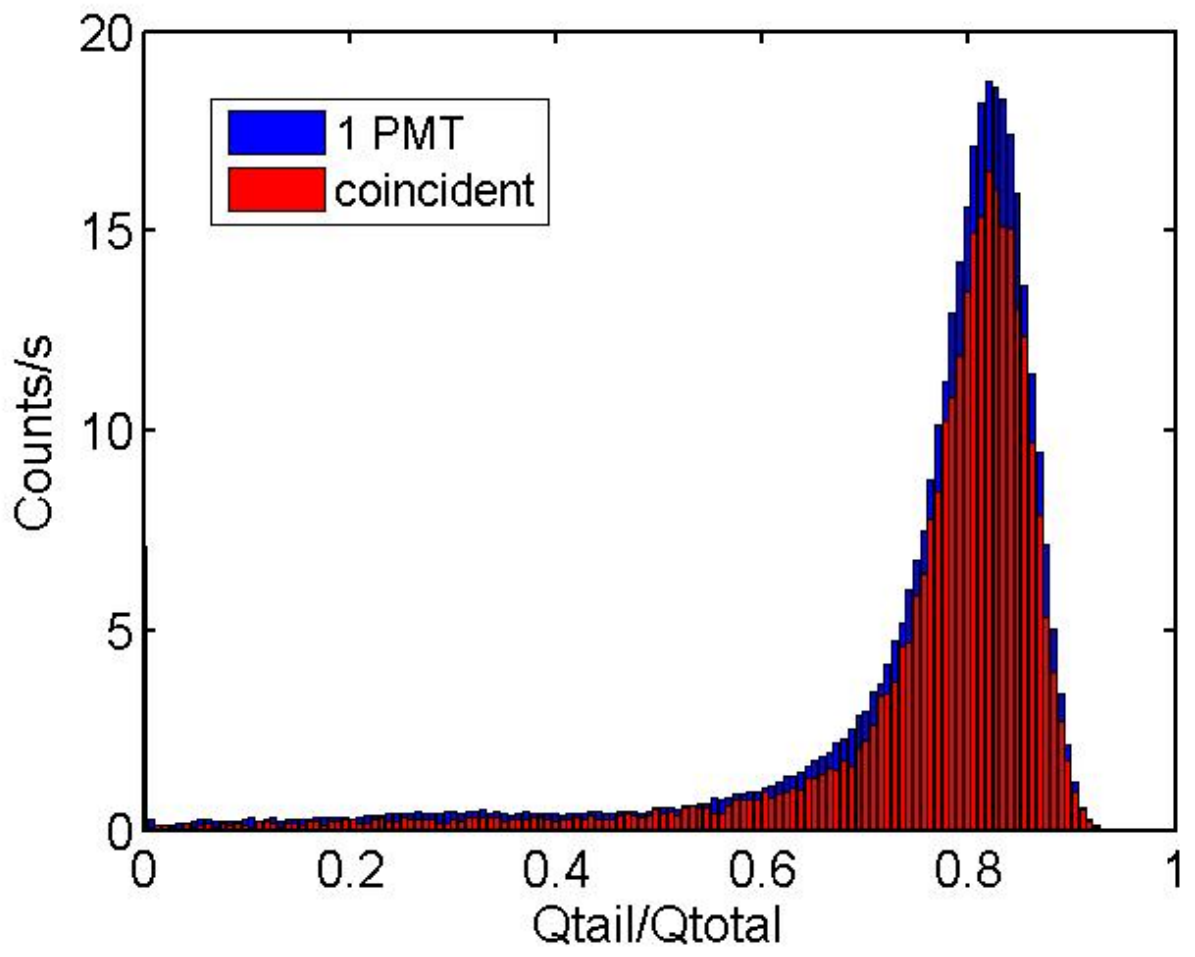

Figure 12. Charge ratio histogram of the traces collected with a ${ }^{252} \mathrm{Cf}$ source positioned in the center of the detector and the 0.7-cm thick WLSP with a single PMT (blue) and with two PMTs in coincidence (red).

Table 4. PMMA and WLSP coincident PMT measurement results with a ${ }^{252} \mathrm{Cf}$ source centered on the detector. The error reported is statistical.

\begin{tabular}{|c|c|}
\hline $\begin{array}{c}\text { Measurement } \\
\text { Configuration }\end{array}$ & $\begin{array}{c}\text { Absolute Neutron } \\
\text { Detection Efficiency }\end{array}$ \\
\hline PMMA ${ }^{252} \mathrm{Cf}(2)$ & $0.0044 \pm 0.0006$ \\
\hline
\end{tabular}


The results of the experiments with the three different thicknesses on the WLSP material are provided in Table 5. The $0.9-\mathrm{cm}$ thick WLSP light guide produced the highest efficiency of the three thicknesses measured, being 15\% higher than with the $0.7-\mathrm{cm}$ thick WLSP light guides. The increase in efficiency is due both to the additional neutron moderation and the improved light transmission. Simulation indicates that perhaps $5 \%$ of the increase is due to the additional moderation, and $10 \%$ is due to improved light collection. The increased thickness provides increased efficiency, but also increases the die-away time. Simulations indicate that the dieaway time will increase $18 \%$ from the $0.7-\mathrm{cm}$ thickness to the $0.9-\mathrm{cm}$ thickness. Overall, the FoM2 (efficiency squared divided by the die-away time) would increase by about $5 \%$ by going to the $0.9-\mathrm{cm}$ thickness. Since this is not a significant effect, the $0.7-\mathrm{cm}$ thickness was chosen for the demonstrator unit. The optimal thickness should be re-evaluated when moving to the fullscale system.

Table 5. Measurement summary for a single PMT coupled directly to the detector with the three different WLSP thicknesses tested. The ${ }^{252} \mathrm{Cf}$ measurements were with the source centered over the detector. The error is dominated by the source activity uncertainty.

\begin{tabular}{|c|c|}
\hline $\begin{array}{c}\text { Measurement } \\
\text { Configuration }\end{array}$ & $\begin{array}{l}\text { Absolute Neutron } \\
\text { Detection Efficiency }\end{array}$ \\
\hline WLSP $0.5 \mathrm{~cm}^{252} \mathrm{Cf}$ & $0.032 \pm 0.005$ \\
\hline WLSP $0.7 \mathrm{~cm}^{252} \mathrm{Cf}$ & $0.040 \pm 0.006$ \\
\hline WLSP $0.9 \mathrm{~cm}^{252} \mathrm{Cf}$ & $0.045 \pm 0.007$ \\
\hline
\end{tabular}

The effect of the tapered light guides on the end of the detector was measured with the $0.7-\mathrm{cm}$ thick WLSP. The light guides added a total of $20 \mathrm{~cm}$ of length $(10 \mathrm{~cm}$ each light guide), increasing the overall size of the system. The neutron detection efficiency with the use of light guides was improved by $38 \%$ for a single PMT, and $17 \%$ for the coincident PMT measurements. The coincident PMT signal improvement estimate is conservative. The detector configuration was modified to accommodate the additional length of the dual light guides, and a correction was applied to the measurements based on comparison measurements between the two configurations, but the correction was conservatively calculated. Based on these measurements, the demonstrator system was designed with the use of tapered light guides between the detector surface and the PMTs. As with the WLSP thickness, the tapered light guides will need to be re-evaluated for the full-scale system, with regards to the overall height of the system and usability.

The gamma-ray sensitivity and discrimination capability were investigated by introducing a gamma-ray source to the detector. In some experiments both the neutron $\left({ }^{252} \mathrm{Cf}\right)$ and gammaray sources were used to ensure that the pulse shape discrimination was not reducing the neutron detection efficiency significantly when a gamma-ray source was present as well. For the WLSP, the gamma-ray response is greatly suppressed when requiring coincidence between the two PMTs (example shown in Figure 13). Since the gamma-ray discrimination is important, and the reduction in efficiency is only $\sim 15 \%$ by requiring coincidence, the demonstrator unit was developed with two PMTs. Both modes (single and coincidence) could then be analyzed and a recommendation for the full-scale system developed on the use of coincidence or not. It may be that the coincidence mode is used when the gamma-ray flux is high (and presumably the neutron rate is also), and a singles mode used when the rates are low. 
PNNL-23011

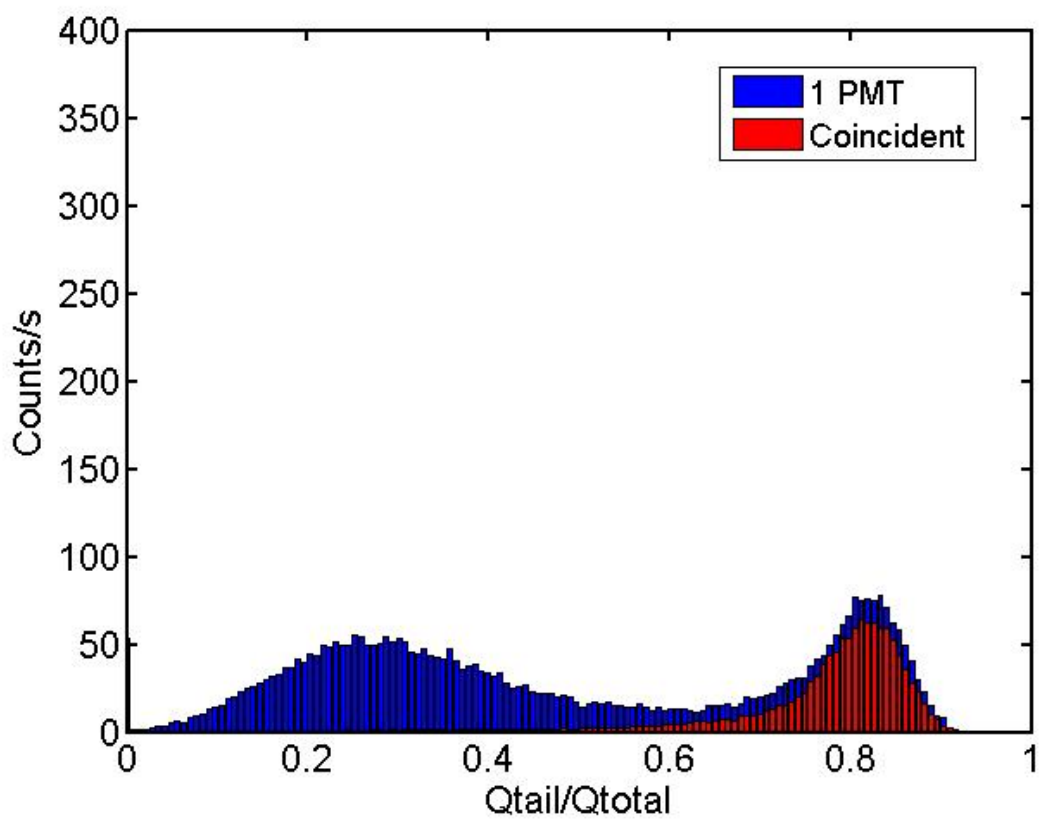

Figure 13. Charge ratio histograms with the 0.7- $\mathrm{cm}$ thick WLSP and a single PMT in response to a ${ }^{252} \mathrm{Cf}$ source $\left(4.0 \times 10^{4} \mathrm{n} / \mathrm{s}\right)$ and an incident gamma-ray flux of $8.5 \times 10^{6}$ with a single PMT (blue) and with two PMTs in coincidence (red).

The gamma-ray discrimination capability was determined by measuring two different gammaray fluxes on the detector for various configurations and data collection modes. The results are shown in Table 6. The PMMA has a better gamma-ray discrimination capability than does the WLSP (which is somewhat gamma-ray sensitive). But as shown before, also has lower neutron detection efficiency. Operating in the coincident mode helps on gamma-ray discrimination significantly for both types of materials.

Table 6. Measurement summary with the 0.7-cm thick PMMA and 0.7- $\mathrm{cm}$ thick WLSP. Coincident measurements are marked with a " $C$ ", the " $\mathrm{H}$ " in parenthesis indicates an incident gamma-ray rate of $5.9 \times 10^{7} \gamma / \mathrm{s}$ and the " $L$ " in parenthesis indicates an incident gamma-ray rate of $8.5 \times 10^{6} \mathrm{\gamma} / \mathrm{s}$. In all cases the source was centered above the detector. The error reported is statistical.

\begin{tabular}{|c|c|}
\hline $\begin{array}{c}\text { Measurement } \\
\text { Configuration }\end{array}$ & $\begin{array}{c}\text { Gamma-Ray } \\
\text { Discrimination }\end{array}$ \\
\hline PMMA $^{137} \mathrm{Cs}(\mathrm{H})$ & $1.1 \times 10^{-5} \pm 1.2 \times 10^{-7}$ \\
\hline PMMA $^{137} \mathrm{Cs}(\mathrm{L})$ & $3.7 \times 10^{-6} \pm 8.8 \times 10^{-8}$ \\
\hline WLSP $^{137} \mathrm{Cs}(\mathrm{H})$ & $1.8 \times 10^{-4} \pm 9.0 \times 10^{-7}$ \\
\hline WLSP $^{137} \mathrm{Cs}(\mathrm{L})$ & $4.8 \times 10^{-5} \pm 5.8 \times 10^{-7}$ \\
\hline PMMA C $^{137} \mathrm{Cs}(\mathrm{H})$ & $8.4 \times 10^{-9} \pm 1.5 \times 10^{-9}$ \\
\hline WLSP C $^{137} \mathrm{Cs}(\mathrm{H})$ & $9.8 \times 10^{-6} \pm 1.3 \times 10^{-7}$ \\
\hline
\end{tabular}

For the PMMA, the coincidence mode increased the discrimination capability by about three orders of magnitude, but also reduced the neutron efficiency by a significant amount. This configuration would probably only be useful in high gamma-ray and neutron flux applications. The WLSP also showed a significant increase in gamma-ray discrimination when used in the coincidence mode, without a large reduction in neutron detection efficiency. Additional gammaray discrimination can be obtained by increasing the threshold for triggering on a pulse, or 
applying a post processing threshold. Gamma rays tend to produce smaller pulses than neutrons and are distributed at low pulse heights; raising the pulse height trigger threshold reduces gamma-rays at a higher rate than neutrons.

One important aspect of the bench-top measurements was to determine the validation correction factor (VCF) for a system using sheets for light guides instead of fibers (as was in the IAT system that was used for the initial VCF estimation). The VCF for the sheet configuration, once determined with the test bench-top unit, could be applied to the full-scale system simulations to obtain a more accurate performance estimate for each of the potential configurations. To calculate the VCF a model of the bench-top unit was constructed. The same simulation methodology as was utilized for the full-scale system simulations was applied to the bench-top unit simulations. The number of neutron captures in the ${ }^{6} \mathrm{LiF}$ was tallied (using a F4 capture tally), but the reaction products were not tracked, nor was the light propagation followed down the light guides. The bench-top model (Figure 14) included the complete bench-top system (six sheets of a plastic light guide, and five sheets ${ }^{6} \mathrm{LiF} / \mathrm{ZnS}$ supported on polyester sheets), the plastic support system, the light-tight box, and the table upon which the unit was positioned. The rest of the room components were far from the detector, and the contributions to the simulated results considered negligible. Each of the three light guide thicknesses measured $(0.5 \mathrm{~cm}, 0.7 \mathrm{~cm}$ and $0.9 \mathrm{~cm})$ were simulated. The measured neutron detection efficiencies for several configurations were compared to the simulated neutron detection efficiencies. The neutron efficiency depends on the pulse height threshold, which in turn affects the gamma-ray discrimination as well as the VCF. An example of the dependency of the gamma-ray rejection and VCF on the pulse height threshold is shown in Figure 15. As the threshold is increased, the gamma-ray rejection improves, but the neutron detection efficiency drops (decreasing the VCF).
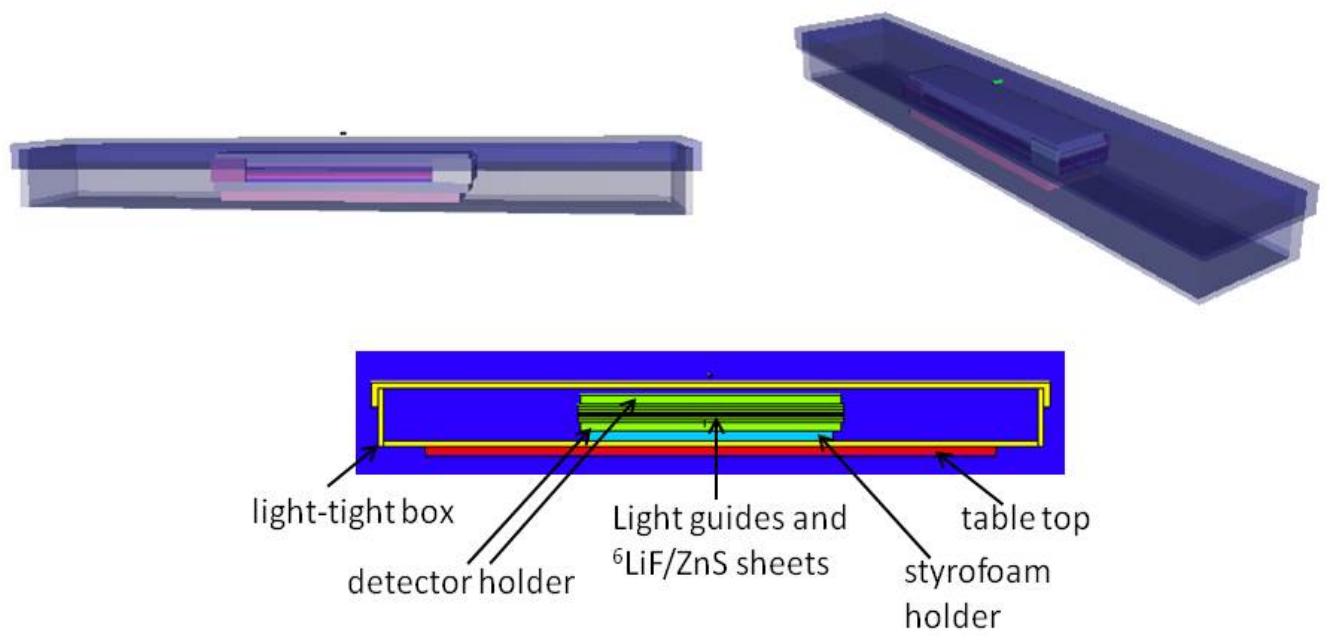

Figure 14. Simulated bench-top detector inside the light tight box (top views) and shown with components labeled in the cross-section view (bottom). 


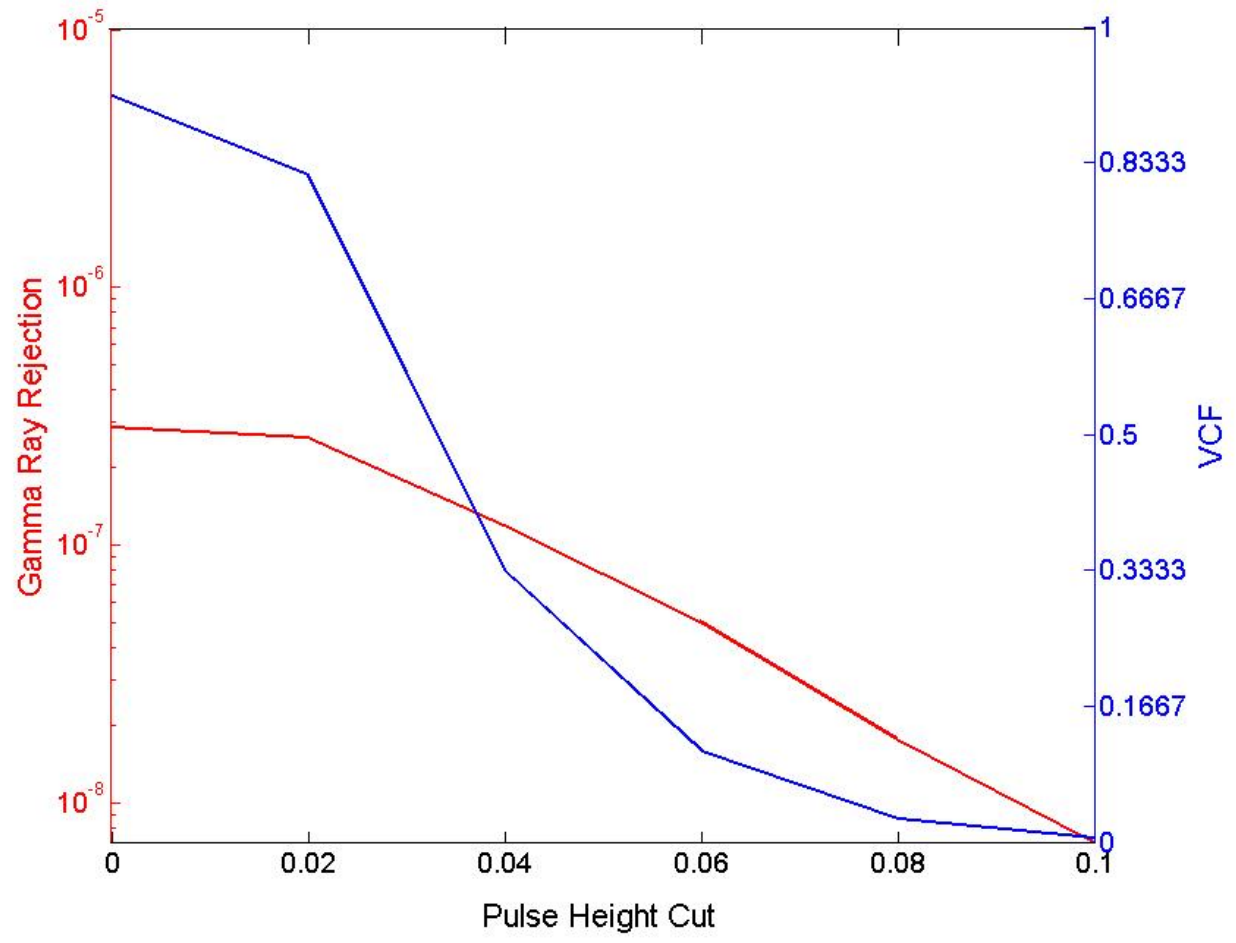

Figure 15. Gamma-ray rejection and VCF for different pulse height thresholds applied to the ${ }^{252} \mathrm{Cf}$ and ${ }^{137} \mathrm{Cs}$ coincidence measurements with the $0.7-\mathrm{cm}$ thick WLSP light guides. 


\section{Gamma-Ray Effects on Mass Estimate}

The LiF/ZnS alternative is somewhat gamma-ray sensitive, as demonstrated in the bench top system, with the sensitivity depending on the light guide used and the various parameter settings such as PMT coincidence requirements or the pulse height threshold. The ${ }^{3} \mathrm{He}$ based systems are fairly immune to gamma rays with typical gamma-ray discrimination (misidentifications) on the order of $10^{-9}$, or one in a billion gamma rays counted as a neutron. The LiF/ZnS bench top paddle system demonstrated a large range of $10^{-4}$ to $10^{-9}$ values. The higher misidentification rates could have significant effects on the mass estimate or assay value of the measurement, depending on the gamma-ray flux. In order to estimate the effect, and determine a reasonable gamma-ray discrimination requirement, an investigation of the effect of gamma-ray misidentification on the mass estimate was undertaken. The effect of gamma-ray detections on the singles, doubles and triples was determined by adding in gamma-ray contributions to the equations used to extract the sample parameters, which were not included previously due to the insignificant gamma-ray sensitivity of ${ }^{3} \mathrm{He}$ proportional tubes.

The time distributions of neutrons are measured at two different places in the pulse stream to estimate the real and accidental multiplicity rates (foreground and background distributions). These multiplicity distributions can be used to unfold the singles, doubles, and triples rates, which then can be used to estimate sample parameters such as the fission rate, the alpha-n rate, and the multiplication in the sample. The fission rate can then be used to estimate the mass of the sample. For the purposes of the following equations, all of the moments are expressed in terms of the source event rate, with the inclusion of $(\alpha, n)$ reactions.

The singles ( $U$ is used here to differentiate from the total source rates $S$ ), doubles $(D)$, and triples $(T)$ for a detector that is only sensitive to neutrons are 15:

$$
\begin{aligned}
& U=F \varepsilon_{n} M v_{S f 1}(1+\alpha) \\
& D=\frac{F f_{d}}{2} \varepsilon_{n}^{2} M^{2}\left(v_{S f 2}+\frac{M-1}{v_{i 1}-1} v_{i 2} v_{s f 1}(1+\alpha)\right) \\
& T=\frac{F f_{t}}{6} \varepsilon_{n}^{3} M^{3}\left[v_{S f 3}+\frac{M-1}{v_{i 1}-1}\left(3 v_{s f 2} v_{i 2}+v_{s f 1}(1+\alpha) v_{i 3}\right)+3\left(\frac{M-1}{v_{i 1}-1}\right)^{2} v_{i 2}^{2} v_{s f 1}(1+\alpha)\right]
\end{aligned}
$$

where

$$
\begin{aligned}
& \begin{array}{l}
v_{s f k}=\text { the factorial moments of the neutrons generated by spontaneous fission } \\
\text { event }
\end{array} \\
& \begin{array}{l}
V_{i k}=\text { the factorial moments of the neutrons generated by an induced fission } \\
\text { event }
\end{array} \\
& F=\text { the spontaneous fission event rate } \\
& S_{\alpha}=\text { the }(\alpha, n) \text { rate }
\end{aligned}
$$




$$
\begin{aligned}
& S=F+S_{\alpha}=\text { the total source event rate } \\
& \alpha=\text { the ratio of }(\alpha, n) \text { to spontaneous fission neutrons }=\frac{S_{\alpha}}{F v_{s f 1}} \\
& M=\text { the sample multiplication (which accounts for additional neutrons in the } \\
& \quad \text { sample due to induced fission) }=\frac{1-p_{i}}{1-p_{i} v_{i 1}} \\
& \varepsilon_{n}=\text { neutron detection efficiency }
\end{aligned}
$$

Note that in these equations the substitutions for the factorial moments of the emitted probability distribution have been made, and $f_{d}$ and $f_{t}$ represent the double and triple gate fractions, respectively.

If significant gamma rays are also detected or misidentified as neutrons and trigger the shift register, these formulas need to be updated to include the gamma-ray contributions. The additions to the equations that must be made to account for the correlated gamma-ray contributions can be seen by starting with the basic forms for the singles, doubles, and triples. The structure of the singles, doubles, and triples equations with only neutron detections considered are (in terms of the factorial moments of the emitted neutron probability distribution, $\left.v_{k}\right)$ :

$$
\begin{aligned}
& U=C_{1} \varepsilon_{n} \nu_{1} \\
& D=C_{2} \varepsilon_{n}^{2} \nu_{2} \\
& T=C_{3} \varepsilon_{n}^{3} v_{3}
\end{aligned}
$$

where $C_{1}, C_{2}$, and $C_{3}$ are simply constants that encompass the source rate, gate fractions, and normalization factors. Each of the equations can be expanded by adding the moments of the gamma-ray distribution $\left(\mu_{k}\right)$, as follows:

$$
\begin{aligned}
& U=C_{1}\left(\varepsilon_{n} \nu_{1}+\varepsilon_{\gamma} \mu_{1}\right) \\
& D=C_{2}\left(\varepsilon_{n}^{2} \nu_{2}+\varepsilon_{\gamma}^{2} \mu_{2}+\varepsilon_{n} \varepsilon_{\gamma} j_{n, \gamma}\right) \\
& T=C_{3}\left(\varepsilon_{n}^{3} \nu_{3}+\varepsilon_{\gamma}^{3} \mu_{3}+\varepsilon_{n}^{2} \varepsilon_{\gamma} j_{n, n, \gamma}+\varepsilon_{n} \varepsilon_{\gamma}^{2} j_{n, \gamma, \gamma}\right)
\end{aligned}
$$

where $\varepsilon_{Y}$ is the gamma-ray detection efficiency and $j_{x, x}$ or $j_{x, x, x}$ are joint moments of the distribution of neutrons $(\mathrm{n})$ and/or gamma-rays $(\mathrm{Y})$.

The factorial moments of the gamma-ray distribution for this study were taken from Pazsit 15 in terms of source events. It is assumed that the gamma rays themselves do not induce additional gamma rays (the gamma-ray chains are non-multiplying); however, gamma rays will be produced as a result of induced fissions along the neutron chain. Therefore, the neutron chains were followed to account for all of these gamma rays. If the sample is not comprised of a pure metal (e.g., the sample is an oxide) a source event could be either spontaneous fission or an $(\alpha, n)$ reaction. Therefore, as with the neutron moments, the moments of the gamma-ray emission probability distribution were weighted to account for the different source events. 
For this initial investigation, only gamma rays produced as a result of either spontaneous or induced fission are considered. One potential source of gamma rays is those released simultaneously with alpha emission; the probability associated with this emission for the isotopes of interest is quite low and neglected here. Other possible sources, such as gamma rays produced from a target nucleus in an excited state or from inelastic neutron scatters are also neglected. Gamma-ray attenuation by the sample itself was not included either. The equation for the first factorial moment of the gamma-ray probability distribution for source events is given as (modified from Pazsit 15):

$$
\mu_{s 1}=\frac{F}{S} \mu_{S f 1}+\frac{F v_{S f 1} \mu_{i 1}(1+\alpha)}{S}\left(\frac{M-1}{v_{i 1}-1}\right)
$$

where $\mu_{s k}=$ the factorial moments of the gamma-ray source distribution,

$\mu_{\text {sfk }}=$ the factorial moments of the gamma-rays generated by spontaneous fission events, and

$\mu_{i k}=$ the factorial moments of the gamma-rays generated by induced fission events.

The following series of diagrams are based on the discussion in Oberer's Thesis 17, but with $(\alpha, n)$ reactions added. The three terms (note that the $(1+\alpha)$ factor produces two terms) in the equation above correspond to the three potential sources of single gamma-rays available for detection, as illustrated below in Figure 16.

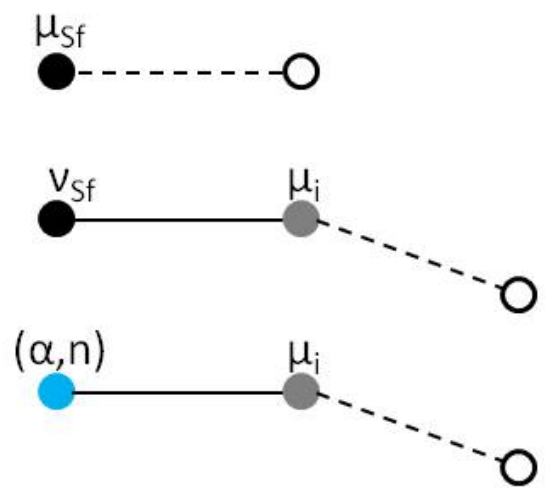

Figure 16. Single gamma-ray sources for the first factorial moment of the gamma-ray probability distribution. The solid lines represent multiplying chains and the dashed lines represent non-multiplying chains. The gray circles represent induced fissions from which a gamma-ray is available for detection (which is represented with an open circle).

The second factorial moment of the gamma-ray probability distribution is: 


$$
\begin{aligned}
& \mu_{S 2}=\frac{F}{S}\left[\mu_{S f 2}+2 \frac{F}{S} \mu_{S f 1} \mu_{i 1} \frac{M-1}{v_{i 1}-1} v_{s f 1}+\right. \\
& \left.v_{s f 2} \mu_{i 1}^{2}\left(\frac{M-1}{v_{i 1}-1}\right)^{2}+v_{s f 1}(1+\alpha) \frac{M-1}{v_{i 1}-1}\left(\mu_{i 2}+2 \mu_{i 1}^{2} v_{i 1} \frac{M-1}{v_{i 1}-1}+v_{i 2} \mu_{i 1}^{2}\left(\frac{M-1}{v_{i 1}-1}\right)^{2}\right)\right]
\end{aligned}
$$

The five source terms correspond to the five potential origins of double gamma rays available for detection as illustrated in Figure 17.
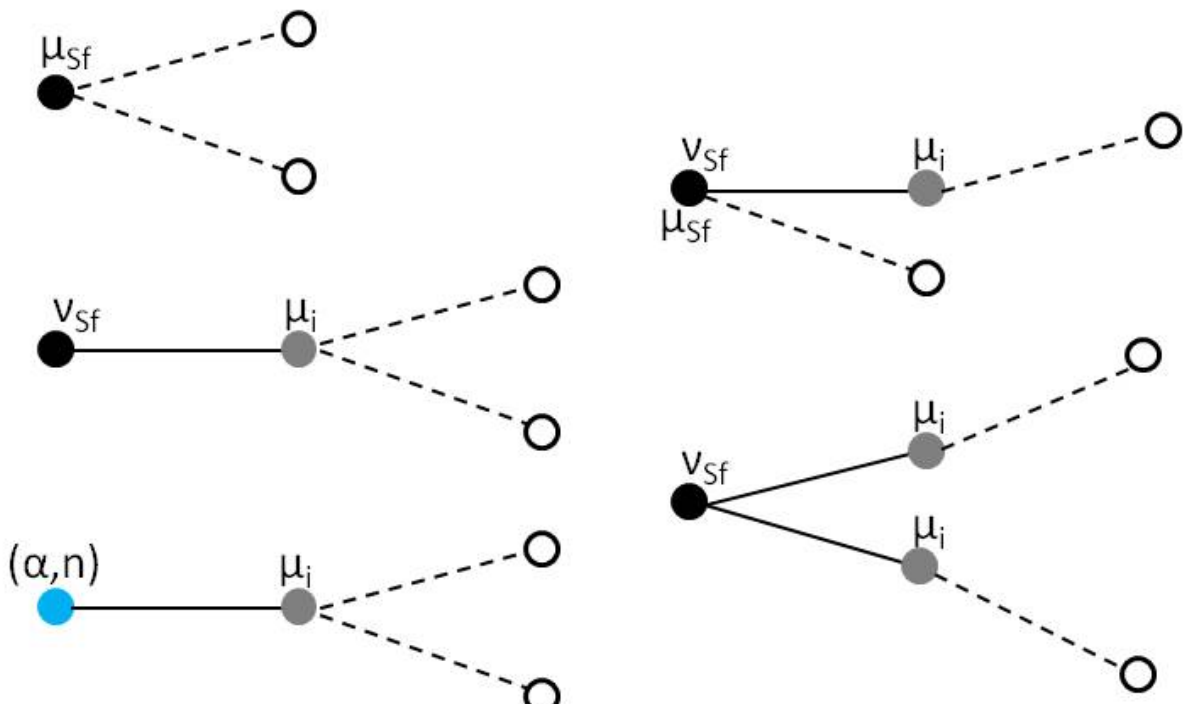

Figure 17. Double gamma-ray sources for the second factorial moment of the gamma-ray probability distribution.

The third factorial moment of the gamma-ray probability distribution is: 


$$
\begin{aligned}
& \mu_{S 3}=\frac{F}{S}\left[\mu_{S f 3}+3 \frac{F}{S} \mu_{S f 2} v_{s f 1} \mu_{i 1} \frac{M-1}{v_{i 1}-1}+3 \mu_{S f 1}\left(v_{s f 2} \mu_{i 1}^{2}\left(\frac{M-1}{v_{i 1}-1}\right)^{2}+v_{s f 1} \frac{M-1}{v_{i 1}-1}\left(\mu_{i 2}+\right.\right.\right. \\
& \left.\left.2 \mu_{i 1}^{2} v_{i 1} \frac{M-1}{v_{i 1}-1}+v_{i 2} \mu_{i 1}^{2}\left(\frac{M-1}{v_{i 1}-1}\right)^{2}\right)\right)+v_{s f 3} \mu_{i 1}^{3}\left(\frac{M-1}{v_{i 1}-1}\right)^{3}+3 v_{s f 2} \mu_{i 1}\left(\frac{M-1}{v_{i 1}-1}\right)^{2}\left(\mu_{i 2}+\right. \\
& \left.2 \mu_{i 1}^{2} v_{i 1} \frac{M-1}{v_{i 1}-1}+v_{i 2} \mu_{i 1}^{2}\left(\frac{M-1}{v_{i 1}-1}\right)^{2}\right)+v_{s f 1}(1+\alpha) \frac{M-1}{v_{i 1}-1}\left(\mu_{i 3}+3 \mu_{i 2} v_{i 1} \mu_{i 1} \frac{M-1}{v_{i 1}-1}+\right. \\
& \left.3 \mu_{i 1}\left(v_{i 2} \mu_{i 1}^{2}\left(\frac{M-1}{v_{i 1}-1}\right)^{2}+v_{i 1} \frac{M-1}{v_{i 1}-1}\left(\mu_{i 2}+2 \mu_{i 1}^{2} v_{i 1} \frac{M-1}{v_{i 1}-1}+v_{i 2} \mu_{i 1}^{2}\left(\frac{M-1}{v_{i 1}-1}\right)^{2}\right)\right)\right]
\end{aligned}
$$

The eight source terms correspond to the eight potential sources of triple gamma rays shown below in Figure 18.

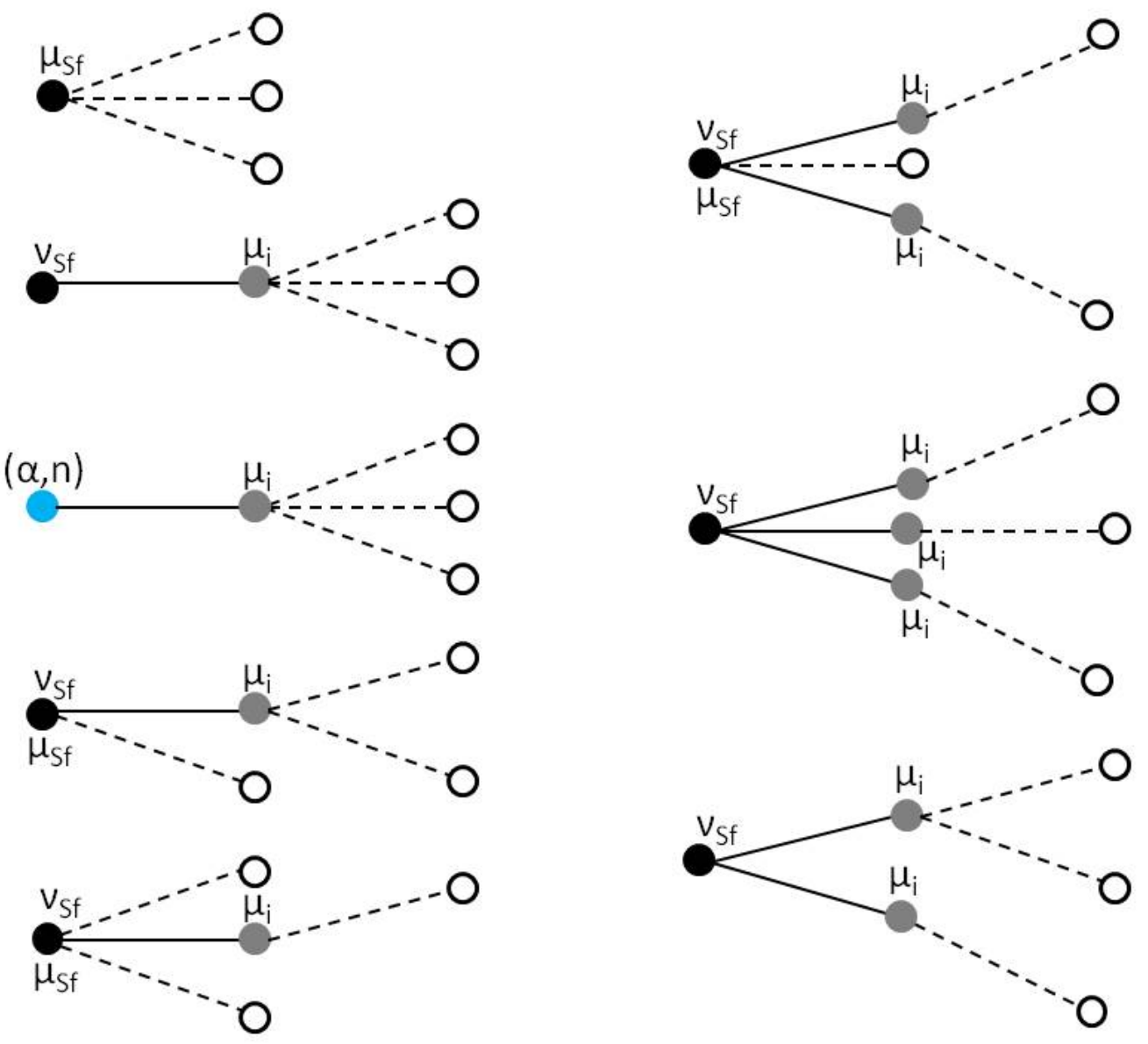

Figure 18. Triple gamma-ray sources for the third factorial moment of the gamma-ray probability distribution.

The joint distributions, or the moments for the probability distribution comprised of neutrons and gamma-rays, were from Pazsit Error! Reference source not found. and Oberer 17, with 
modifications to include the effect of $(\alpha, n)$ reactions and to be consistently expressed in terms of the source rate. The joint moment of interest for the doubles rate is $j_{n, y}$, which represents the moment of the distribution of joint neutron and gamma-ray quanta that would make a neutron and a gamma-ray available for detection.

$$
j_{n \gamma}=\frac{F}{S}\left(\mu_{S f 1} \frac{F}{S} v_{s f 1} M+M \mu_{i 1} \frac{M-1}{v_{i 1}-1} v_{s f 2}+\frac{M-1}{v_{i 1}-1} v_{s f 1}(1+\alpha)\left(\mu_{i 1} v_{i 1} M+M \mu_{i 1} \frac{M-1}{v_{i 1}-1} v_{i 2}\right)\right)
$$

Due to the complexity of the images corresponding diagrams are not included here for the joint moments.

Two joint moments are required for the triples expression; one for the distribution that would make two neutrons and one gamma-ray available for detection, $j_{n n y}$, and one for the distribution that would make one neutron and two gamma-rays available for detection, $j_{n \gamma \gamma}$.

$$
\begin{aligned}
& j_{n n \gamma}=\frac{F}{S}\left[\mu_{S f 1}\left(\frac{F}{S} v_{s f 2} M^{2}+\frac{F}{S} v_{s f 1} \frac{M-1}{v_{i 1}-1} v_{i 2} M^{2}\right)+v_{s f 3} \mu_{i 1} \frac{M-1}{v_{i 1}-1} M^{2}+\right. \\
& v_{s f 2}\left(2 M \frac{M-1}{v_{i 1}-1}\left(\mu_{i 1} v_{i 1} M+v_{i 2} M \mu_{i 1} \frac{M-1}{v_{i 1}-1}\right)+\mu_{i 1}\left(\frac{M-1}{v_{i 1}-1}\right)^{2} v_{i 2} M^{2}\right)+ \\
& v_{s f 1}(1+\alpha) \frac{M-1}{v_{i 1}-1}\left(\mu_{i 1}\left(v_{i 2} M^{2}+v_{i 1} v_{i 2} \frac{M-1}{v_{i 1}-1} M^{2}\right)+v_{i 3} M^{2} \mu_{i 1} \frac{M-1}{v_{i 1}-1}+\right. \\
& \left.\left.v_{i 2}\left(v_{i 2} \mu_{i 1}\left(\frac{M-1}{v_{i 1}-1}\right)^{2} M^{2}+2 M \frac{M-1}{v_{i 1}-1}\left(\mu_{i 1} v_{i 1} M+v_{i 2} M \mu_{i 1} \frac{M-1}{v_{i 1}-1}\right)\right)\right)\right]
\end{aligned}
$$

And:

$$
\begin{aligned}
& j_{n \gamma \gamma}=\frac{F}{S}\left[\mu_{S f 2} \frac{F}{S} v_{s f 1} M+2 \mu_{S f 1}\left(\frac{F}{S} v_{s f 2} M \mu_{i 1} \frac{M-1}{v_{i 1}-1}+\frac{F}{S} v_{s f 1} \frac{M-1}{v_{i 1}-1}\left(\mu_{i 1} v_{i 1} M+\right.\right.\right. \\
& \left.\left.v_{i 2} M \mu_{i 1} \frac{M-1}{v_{i 1}-1}\right)\right)+v_{s f 3} M \mu_{i 1}^{2}\left(\frac{M-1}{v_{i 1}-1}\right)^{2}+v_{s f 2}\left(M \frac { M - 1 } { v _ { i 1 } - 1 } \left(\mu_{i 2}+2 \mu_{i 1}^{2} v_{i 1} \frac{M-1}{v_{i 1}-1}+\right.\right. \\
& \left.\left.v_{i 2} \mu_{i 1}^{2}\left(\frac{M-1}{v_{i 1}-1}\right)^{2}\right)+2 \mu_{i 1}\left(\frac{M-1}{v_{i 1}-1}\right)^{2}\left(\mu_{i 1} v_{i 1} M+v_{i 2} M \mu_{i 1} \frac{M-1}{v_{i 1}-1}\right)\right)+ \\
& v_{s f 1}(1+\alpha) \frac{M-1}{v_{i 1}-1}\left(\mu_{i 2} v_{i 1} M+2 \mu_{i 1}\left(v_{i 2} M \mu_{i 1} \frac{M-1}{v_{i 1}-1}+v_{i 1} \frac{M-1}{v_{i 1}-1}\left(\mu_{i 1} v_{i 1} M+v_{i 2} M \mu_{i 1} \frac{M-1}{v_{i 1}-1}\right)\right)+\right. \\
& v_{i 3} M \mu_{i 1}^{2}\left(\frac{M-1}{v_{i 1}-1}\right)^{2}+v_{i 2}\left(2 \mu_{i 1}\left(\frac{M-1}{v_{i 1}-1}\right)^{2}\left(\mu_{i 1} v_{i 1} M+v_{i 2} M \mu_{i 1} \frac{M-1}{v_{i 1}-1}\right)+\right. \\
& \left.\left.M \frac{M-1}{v_{i 1}-1}\left(\mu_{i 2}+2 \mu_{i 1}^{2} v_{i 1} \frac{M-1}{v_{i 1}-1}+v_{i 2} \mu_{i 1}^{2}\left(\frac{M-1}{v_{i 1}-1}\right)^{2}\right)\right)\right]
\end{aligned}
$$

The neutron, gamma-ray and joint moments can now be inserted to produce equations for the detected and counted singles, doubles and triples as follows. 
Singles:

$$
U=S\left[\varepsilon_{n} M \frac{F}{S} v_{S f 1}(1+\alpha)+\varepsilon_{\gamma} \frac{F}{S}\left(\mu_{S f 1}+v_{S f 1} \mu_{i 1} \frac{M-1}{v_{i 1}-1}(1+\alpha)\right)\right]
$$

Doubles:

$$
\begin{aligned}
& D=\frac{S f_{d}}{2}\left[\varepsilon_{n}^{2} \frac{F}{S} M^{2}\left(v_{S f 2}+\frac{M-1}{v_{i 1}-1} v_{i 2} v_{s f 1}(1+\alpha)\right)+\varepsilon_{\gamma}^{2} \frac{F}{S}\left(\mu_{S f 2}+2 \frac{F}{S} \mu_{S f 1} \mu_{i 1} \frac{M-1}{v_{i 1}-1} v_{s f 1}+\right.\right. \\
& \left.v_{s f 2} \mu_{i 1}^{2}\left(\frac{M-1}{v_{i 1}-1}\right)^{2}+v_{s f 1}(1+\alpha) \frac{M-1}{v_{i 1}-1}\left(\mu_{i 2}+2 \mu_{i 1}^{2} v_{i 1} \frac{M-1}{v_{i 1}-1}+v_{i 2} \mu_{i 1}^{2}\left(\frac{M-1}{v_{i 1}-1}\right)^{2}\right)\right)+ \\
& \left.\varepsilon_{n} \varepsilon_{\gamma} \frac{F}{S}\left(\mu_{S f 1} \frac{F}{S} v_{s f 1} M+M \mu_{i 1} \frac{M-1}{v_{i 1}-1} v_{s f 2}+\frac{M-1}{v_{i 1}-1} v_{s f 1}(1+\alpha)\left(\mu_{i 1} v_{i 1} M+M \mu_{i 1} \frac{M-1}{v_{i 1}-1} v_{i 2}\right)\right)\right]
\end{aligned}
$$

And triples: 


$$
\begin{aligned}
& T=\frac{S f_{t}}{6}\left[\varepsilon_{n}^{3}\left[M^{3} \frac{F}{S}\left(v_{S f 3}+\frac{M-1}{v_{i 1}-1}\left(3 v_{s f 2} v_{i 2}+v_{s f 1}(1+\alpha) v_{i 3}\right)+3\left(\frac{M-1}{v_{i 1}-1}\right)^{2} v_{i 2}^{2} v_{s f 1}(1+\alpha)\right)\right]+\right. \\
& \varepsilon_{\gamma}^{3}\left[\frac { F } { S } \left(\mu_{S f 3}+3 \frac{F}{S} \mu_{S f 2} v_{s f 1} \mu_{i 1} \frac{M-1}{v_{i 1}-1}+3 \mu_{S f 1}\left(v_{s f 2} \mu_{i 1}^{2}\left(\frac{M-1}{v_{i 1}-1}\right)^{2}+v_{s f 1} \frac{M-1}{v_{i 1}-1}\left(\mu_{i 2}+\right.\right.\right.\right. \\
& \left.\left.2 \mu_{i 1}^{2} v_{i 1} \frac{M-1}{v_{i 1}-1}+v_{i 2} \mu_{i 1}^{2}\left(\frac{M-1}{v_{i 1}-1}\right)^{2}\right)\right)+v_{s f 3} \mu_{i 1}^{3}\left(\frac{M-1}{v_{i 1}-1}\right)^{3}+3 v_{s f 2} \mu_{i 1}\left(\frac{M-1}{v_{i 1}-1}\right)^{2}\left(\mu_{i 2}+\right. \\
& \left.2 \mu_{i 1}^{2} v_{i 1} \frac{M-1}{v_{i 1}-1}+v_{i 2} \mu_{i 1}^{2}\left(\frac{M-1}{v_{i 1}-1}\right)^{2}\right)+v_{s f 1}(1+\alpha) \frac{M-1}{v_{i 1}-1}\left(\mu_{i 3}+3 \mu_{i 2} v_{i 1} \mu_{i 1} \frac{M-1}{v_{i 1}-1}+\right. \\
& \left.\left.3 \mu_{i 1}\left(v_{i 2} \mu_{i 1}^{2}\left(\frac{M-1}{v_{i 1}-1}\right)^{2}+v_{i 1} \frac{M-1}{v_{i 1}-1}\left(\mu_{i 2}+2 \mu_{i 1}^{2} v_{i 1} \frac{M-1}{v_{i 1}-1}+v_{i 2} \mu_{i 1}^{2}\left(\frac{M-1}{v_{i 1}-1}\right)^{2}\right)\right)\right)\right]+ \\
& \varepsilon_{n}^{2} \varepsilon_{\gamma} \frac{F}{S}\left[\mu_{S f 1}\left(\frac{F}{S} v_{s f 2} M^{2}+\frac{F}{S} v_{s f 1} \frac{M-1}{v_{i 1}-1} v_{i 2} M^{2}\right)+v_{s f 3} \mu_{i 1} \frac{M-1}{v_{i 1}-1} M^{2}+\right. \\
& v_{s f 2}\left(2 M \frac{M-1}{v_{i 1}-1}\left(\mu_{i 1} v_{i 1} M+v_{i 2} M \mu_{i 1} \frac{M-1}{v_{i 1}-1}\right)+\mu_{i 1}\left(\frac{M-1}{v_{i 1}-1}\right)^{2} v_{i 2} M^{2}\right)+ \\
& v_{s f 1}(1+\alpha) \frac{M-1}{v_{i 1}-1}\left(\mu_{i 1}\left(v_{i 2} M^{2}+v_{i 1} v_{i 2} \frac{M-1}{v_{i 1}-1} M^{2}\right)+v_{i 3} M^{2} \mu_{i 1} \frac{M-1}{v_{i 1}-1}+\right. \\
& \left.\left.v_{i 2}\left(v_{i 2} \mu_{i 1}\left(\frac{M-1}{v_{i 1}-1}\right)^{2} M^{2}+2 M \frac{M-1}{v_{i 1}-1}\left(\mu_{i 1} v_{i 1} M+v_{i 2} M \mu_{i 1} \frac{M-1}{v_{i 1}-1}\right)\right)\right)\right]+ \\
& \varepsilon_{n} \varepsilon_{\gamma}^{2} \frac{F}{S}\left[\mu_{S f 2} \frac{F}{S} v_{s f 1} M+2 \mu_{S f 1}\left(\frac{F}{S} v_{s f 2} M \mu_{i 1} \frac{M-1}{v_{i 1}-1}+\frac{F}{S} v_{s f 1} \frac{M-1}{v_{i 1}-1}\left(\mu_{i 1} v_{i 1} M+\right.\right.\right. \\
& \left.\left.v_{i 2} M \mu_{i 1} \frac{M-1}{v_{i 1}-1}\right)\right)+v_{s f 3} M \mu_{i 1}^{2}\left(\frac{M-1}{v_{i 1}-1}\right)^{2}+v_{s f 2}\left(M \frac { M - 1 } { v _ { i 1 } - 1 } \left(\mu_{i 2}+2 \mu_{i 1}^{2} v_{i 1} \frac{M-1}{v_{i 1}-1}+\right.\right. \\
& \left.\left.v_{i 2} \mu_{i 1}^{2}\left(\frac{M-1}{v_{i 1}-1}\right)^{2}\right)+2 \mu_{i 1}\left(\frac{M-1}{v_{i 1}-1}\right)^{2}\left(\mu_{i 1} v_{i 1} M+v_{i 2} M \mu_{i 1} \frac{M-1}{v_{i 1}-1}\right)\right)+ \\
& v_{s f 1}(1+\alpha) \frac{M-1}{v_{i 1}-1}\left(\mu_{i 2} v_{i 1} M+2 \mu_{i 1}\left(v_{i 2} M \mu_{i 1} \frac{M-1}{v_{i 1}-1}+v_{i 1} \frac{M-1}{v_{i 1}-1}\left(\mu_{i 1} v_{i 1} M+\right.\right.\right. \\
& \left.\left.v_{i 2} M \mu_{i 1} \frac{M-1}{v_{i 1}-1}\right)\right)+v_{i 3} M \mu_{i 1}^{2}\left(\frac{M-1}{v_{i 1}-1}\right)^{2}+v_{i 2}\left(2 \mu_{i 1}\left(\frac{M-1}{v_{i 1}-1}\right)^{2}\left(\mu_{i 1} v_{i 1} M+v_{i 2} M \mu_{i 1} \frac{M-1}{v_{i 1}-1}\right)+\right. \\
& \left.\left.\left.\left.M \frac{M-1}{v_{i 1}-1}\left(\mu_{i 2}+2 \mu_{i 1}^{2} v_{i 1} \frac{M-1}{v_{i 1}-1}+v_{i 2} \mu_{i 1}^{2}\left(\frac{M-1}{v_{i 1}-1}\right)^{2}\right)\right)\right)\right]\right]
\end{aligned}
$$


If $\varepsilon_{y}=0$ the above equations collapse back to the original forms containing neutron moments only. It should be noted that these equations assume that the neutron and gamma-ray die-away times are the same. The die-away time depends on the detector design, but it is unlikely to be the same for neutrons and gamma rays. The effect of different die-away times could be considered in future work.

The effect of the gamma-ray detection (and misidentified as a neutron) was calculated using the above equations to determine the bias in the mass estimate. The effect on the calculated mass for different values of $M$ and $\alpha$ is shown in below in Figure 19, for a detector with the same parameters as the initial simulated ${ }^{6} \mathrm{LiF} / \mathrm{ZnS}$ based multiplicity counter (a neutron detection efficiency of $43 \%$ and a linear die-away time of $8 \mu \mathrm{s}$ ) and using a 10-g mass of plutonium-240. The data was generated by calculating the singles, doubles and triples for a range of gammaray efficiencies from zero to one (100 percent). It should be noted that the calculated values for $M$ and $\alpha$ also change when $\varepsilon_{\mathrm{Y}}>0$, but those dependencies are not shown in this figure. The impact of correlated gamma rays being detected and counted as part of the correlated events can be significant, and will vary depending on the parameters of the sample being assayed.

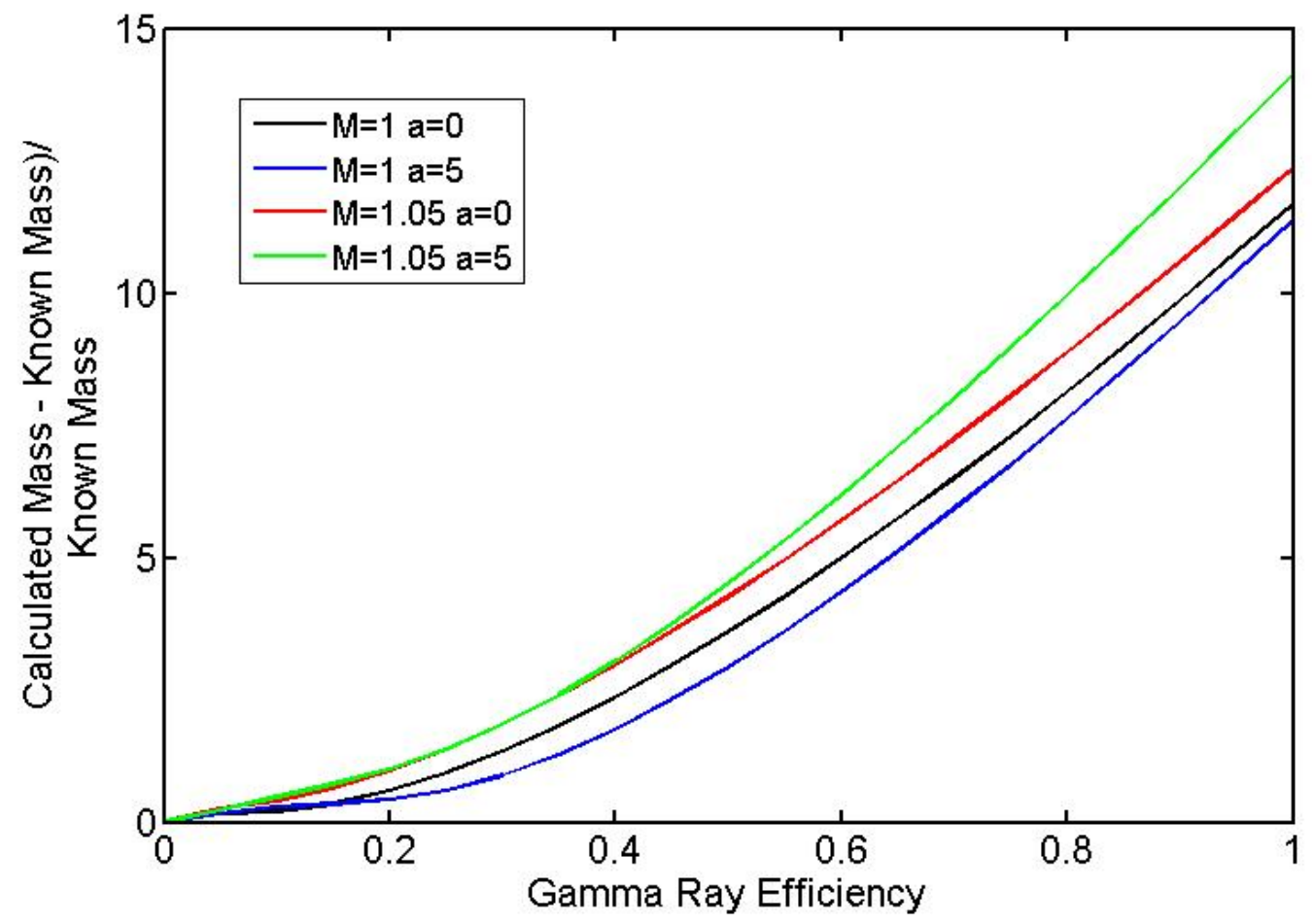

Figure 19. The effect of the gamma-ray efficiency on the calculated mass for a $10 \mathrm{~g}{ }^{240} \mathrm{Pu}$ sample with different values of $M$ and $\alpha$ (a).

The PSD criteria applied during post-processing minimize the effect of the gamma-ray efficiency. Therefore, the effective gamma-ray efficiency of the system is less than the gammaray efficiency of the detector itself. The effect of the correlated gamma-ray moments on the calculated mass was plotted for a more reasonable range of gamma-ray rejection levels in Figure 20 below. Note that the effect is larger when $\alpha=0$. This is due to the fact that for $\alpha>0$ there is a larger contribution to the singles rates from the neutron moments than the gamma-ray moments (at low gamma-ray efficiencies), which produces a smaller discrepancy in the calculated mass value than with simulations where $\alpha=0$. 


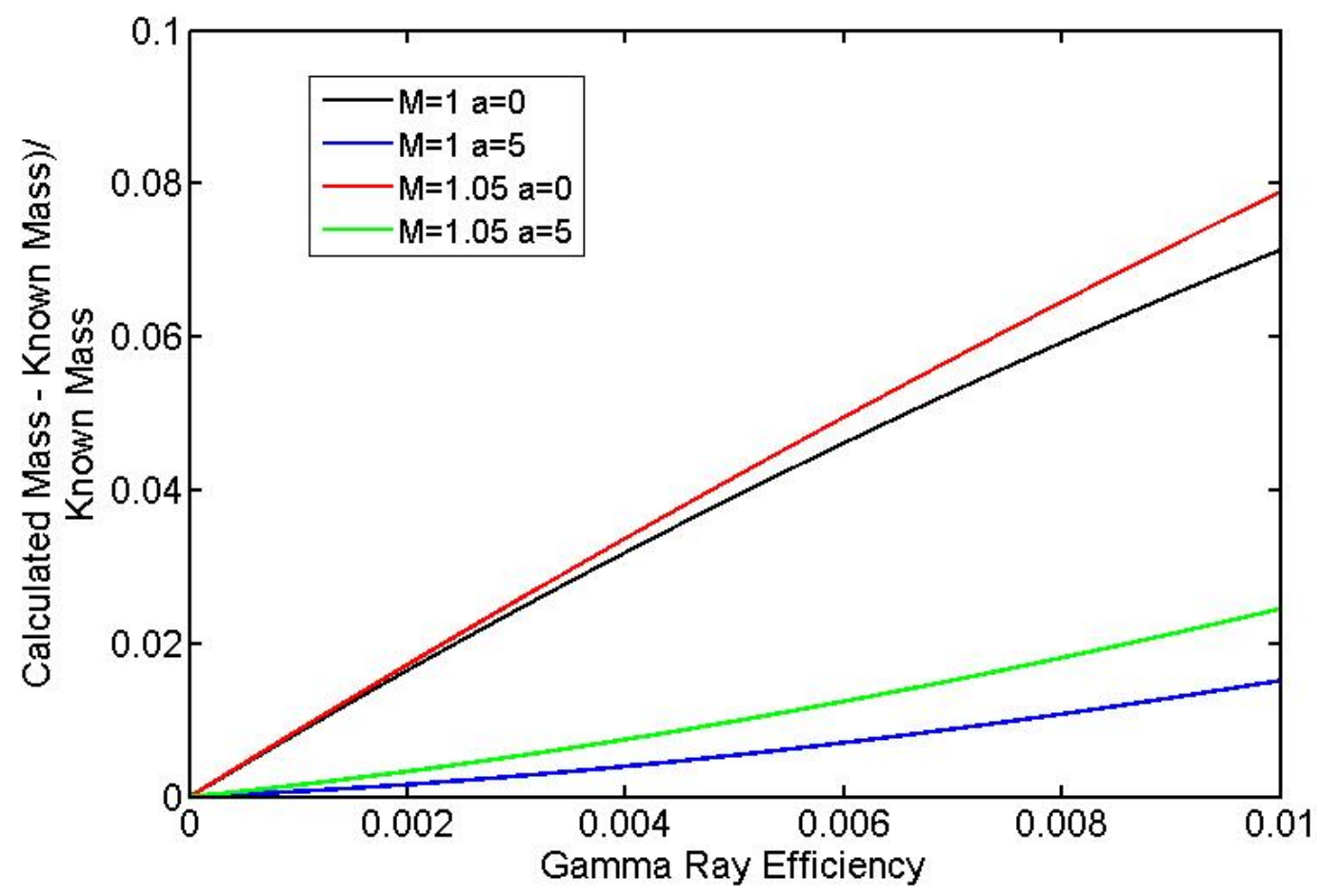

Figure 20. Detail of Error! Reference source not found. for the expected gamma-ray efficiency of the ${ }^{6} \mathrm{LiF} / \mathrm{ZnS}$ based bench-top system.

A closed form solution for $\mathrm{M}, \mathrm{F}$ and $\alpha$ when the gamma-ray distributions are included in the equations for the singles, doubles and triples would be non-trivial to obtain, since it would require solving an equation in the fifth power for the multiplication (M). However, solutions for $\mathrm{M}, \mathrm{F}$ and $\alpha$ can be generated for measured singles, doubles and triples by solving the equations iteratively using a least squares method to compare the measured $U$, $D$ and $T$ to calculated values.

Initial measurements with the test unit demonstrated that a gamma-ray rejection below $10^{-5}$ could be achieved with the unit operated in coincidence mode and PSD applied. For a gammaray rejection of $10^{-5}$, the effect of the gamma-ray moments on the calculated mass would be approximately $0.009 \%$ as simulated for a sample with $M=1$ and $\alpha=0$. This is a relatively small error compared to the desired $1 \%$ estimate on the mass from the measurement where the uncertainty is dominated by the statistics. However, this exploration was a simulation exercise, and would need to be verified with experimental measurements with a variety of sources with different multiplication and alpha values. 
PNNL-23011

\section{Pile-up and Pulse Shape Discrimination}

As the event rate increases, events will become piled-up, that is, another pulse arrives before the first pulse has disappeared. The $\mathrm{ZnS}: \mathrm{Ag}$ has a long luminescence lifetime and the response generated from a neutron can be on the order of several microseconds long as was shown previously in Error! Reference source not found. For event rates from several hundred $\mathrm{kHz}$ to a $\mathrm{MHz}$, pulses will pile-up and the neutron signatures will become more difficult to discriminate from gamma-rays. In particular, a traditional Pulse Shape Discrimination approach using the ratio of the total signal within a short and long time window becomes untenable, as these windows may be occupied by multiple confounding signatures. Multiplicity counting requires an approach capable of detection of neutron events without obfuscation by gamma-ray or other neutron events, or lost signatures due to pileup. To achieve this at high total event rate requires a time-filtering technique able to discriminate between neutrons and gamma-rays over a short time scale. An exploration of filtering techniques was undertaken to be able to separate piledup pulses from each other, and at the same time, to separate neutron and gamma-ray responses.

The initial approach was a wavelet filtering algorithm designed to recognize neutron events in a minimum amount of time. Ideally, wavelets are employed that bear similarity to the expected data features. The wavelet filter applied to a time series of data $f(x)$ takes the form

$\left[W_{\varphi}\right](a, b)=\frac{1}{\sqrt{|a|}} \int_{-\infty}^{\infty} \varphi \frac{x-b}{a} f(x) d x$

where $\varphi$ is a wavelet filter function chosen for its response to expected data features, and $a$ is a scale factor representing the "width" of the filter. Wavelet filters are often used to describe variations in a data series and to identify expected data signatures [18-19]. The use of zeronormed filters is appropriate in data where a nonzero baseline count rate is expected (as with piled-up signal data). While many zero-normed filters are possible and a rigorous optimization was beyond the scope of this work, the desired filter has the same time-scale and features as a neutron event, producing a high correlation when the data and filter are similar, while retaining a simple form for easy comparison across data sets. The Haar wavelet function was chosen for these reasons, and consists of a flat maximum paired with an equal negative region, providing a simple structure that can be scaled in time while maintaining a zero norm.

In order to evaluate the effectiveness of the filtering techniques for identifying neutron signatures in cases of significant pileup, synthetic data representing piled-up gamma-ray and neutron events were produced by juxtaposing and summing the data from measured events. For each case, the filter was then applied over the data. A filter size was optimized to maximize the response to matched events (i.e., neutrons) and minimize the response to data features on any other size scale (i.e., gamma-rays and noise). A filter width of 200 time steps using the XIA data acquisition module at $75 \mathrm{MHz}$ (corresponding to a positive region in the Haar filter function of around $1.3 \mu \mathrm{s}$ ) was used to provide a filter designed to enhance events of similar width and suppress events of shorter width (Figure 21). 

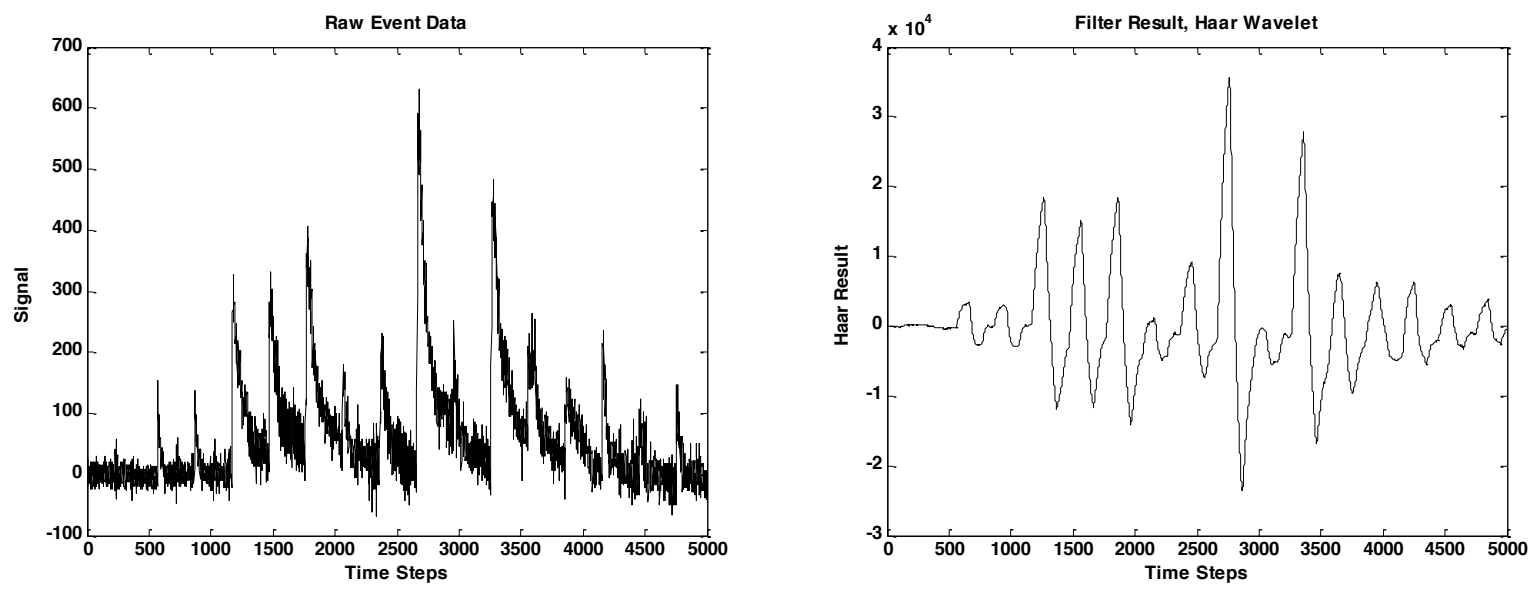

Figure 21. Synthetically piled-up data and Haar filter output.

To provide sufficient resolution of events, a multi-step algorithm was implemented using the raw data and Haar-filtered data. First, each local maximum in the filtered data was identified to produce a list of estimated event locations. These maxima were defined as each location that represented a maximum value within a 200 -timestep window. These events were compared with a threshold and rejected at a level sufficiently high to exclude most noise. This initial stage allowed for sensitive evaluation of only those locations in the filtered data that are likely to correspond to gamma-ray or neutron events. Each event in the filtered data was then associated with a local maximum in the raw data. The ratio of the two peaks (the peak of the Haar-filtered data and the peak of the raw data) was collected for each event. Synthetically piled-up events with varying time spacing were examined using a single filter width (200 time steps) to determine the dependence of the threshold on pileup. Examples with four different event spacings between 200 and 500 time steps are shown below in Figure 22. The optimal ratio to use as a threshold does not change significantly as pileup increases. However, the degree to which gamma-ray and neutron events can be separated depends strongly on the degree of pileup. 

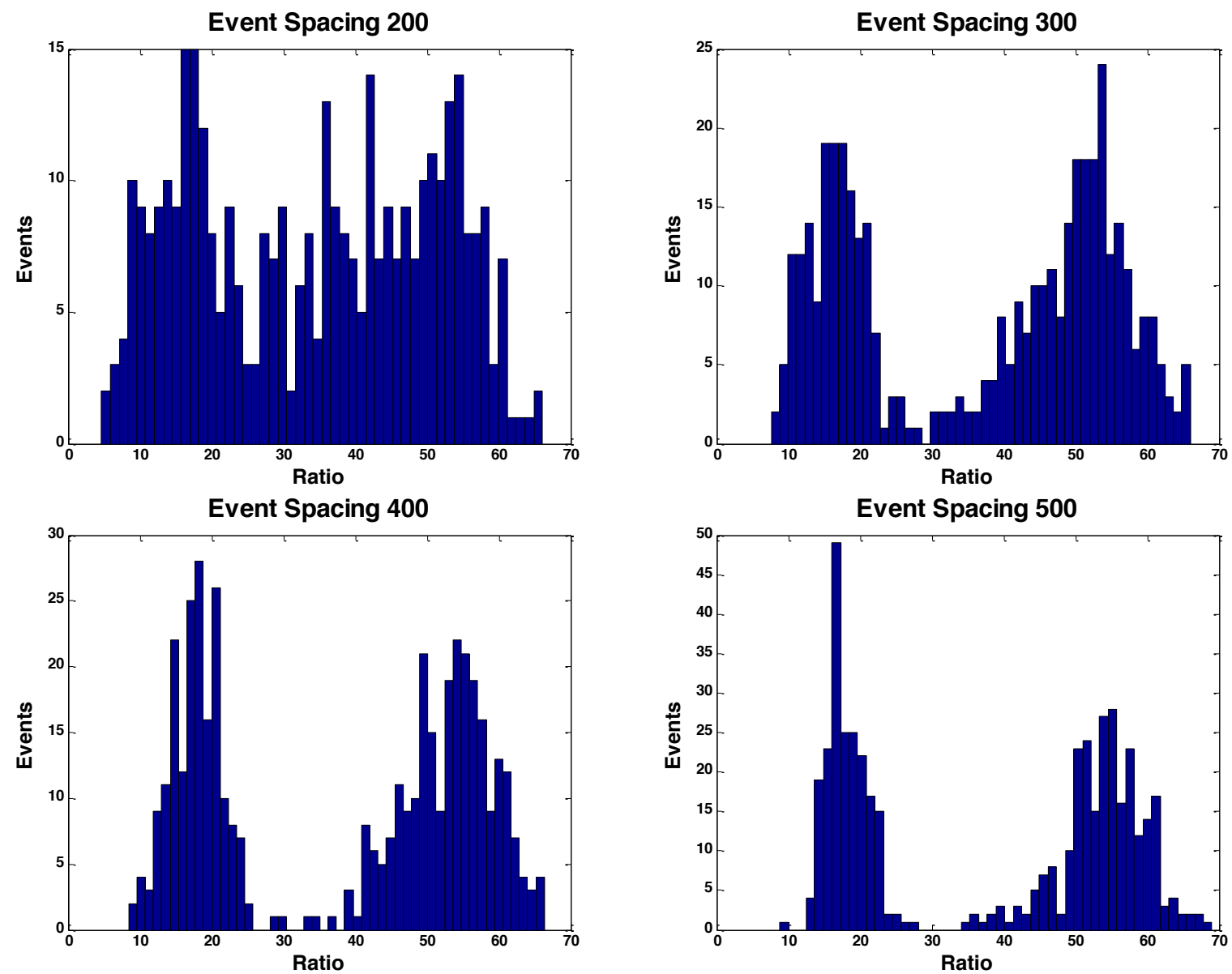

Figure 22. Ratio of Haar to raw event amplitudes for varying events spacing (degree of piled-up events).

Event separation was further estimated by establishing a "ground truth" by employing the Haar filtering algorithm on well-separated events, and then using this ground truth to establish algorithm performance under pile-up. The identified events were held for comparison with the results from the same analysis performed on piled-up data (the same data summed together with shorter time spacing), and the events in the resulting synthetic data labeled as neutrons or gamma rays (Figure 23).

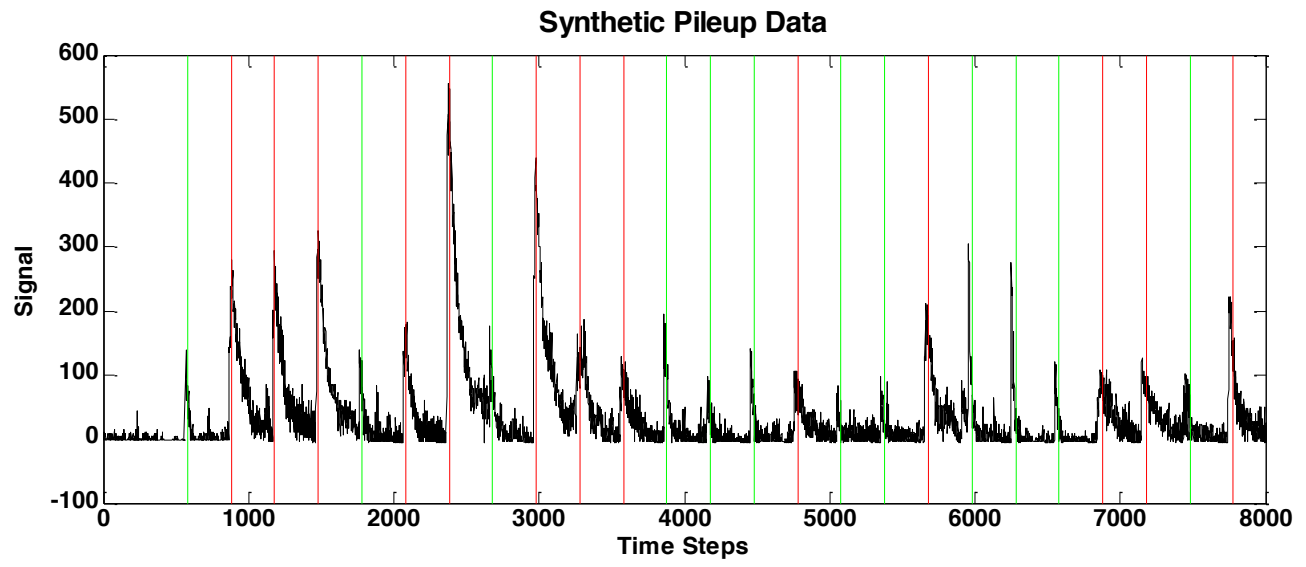

Figure 23. Color-coded neutron (red) and gamma-ray (green) events, 3.9- $\mu$ s event standoff (0.26 MHz). 
The estimated ground truth data was used to determine the performance for a large data set as a function of pile-up. The performance was calculated for the data when requiring coincidence between the two PMTs of the detector, as well as for all events (no coincidence required) to understand the effect of the detector configuration on the signals produced.

The probability of neutron detection was calculated as the ratio of estimated neutron events in the piled-up data sample to actual neutron events. This quantity was computed and plotted with respect to the pile-up offset in Figure 24. These results suggest a falloff in neutron detection when the spacing between events is less than $4 \mu \mathrm{s}$. Only a slight enhancement to the neutron count rate is seen when using the coincidence data, between 5 and $10 \%$ of the total, due to the enhancement in counting statistics as a result of including both traces in a coincidence measurement.

Since the die-away time of the full-scale system is estimated from the modeling and simulation results to be on the order of $10 \mu \mathrm{s}$, and the expected rate of total events for the whole system is in the range of $1 \mathrm{MHz}$, the pile-up algorithm needs to have better performance than the wavelet filter results, and research into alternative approaches was conducted.

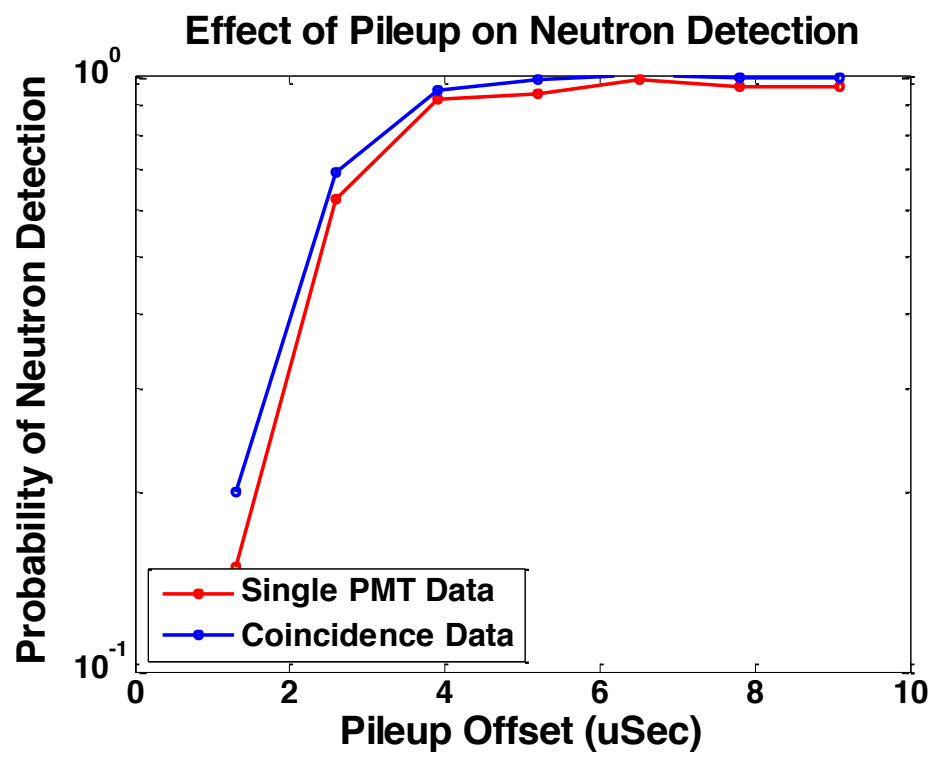

Figure 24. Neutron detections under pileup conditions.

The next approach was an iterative regressive approach, developed to further enhance neutron identification efficiency. In this approach, filters were first used to establish the locations of pulses in the data stream, after which the height, length and type of each pulse was used to establish the optimal fit to the data using exemplar response functions for neutron and gammaray signals. This multi-stage event analysis begins with a development of the exemplar response function for neutron and gamma-ray signals from the detector. Signatures for neutron and gamma-ray events were constructed by summing (and then normalizing) these sorts of events from actual data, with examples shown in Figure 25. In future implementations, these signatures will be constructed from knowledge of the detector and electronics, and validated against real data. 

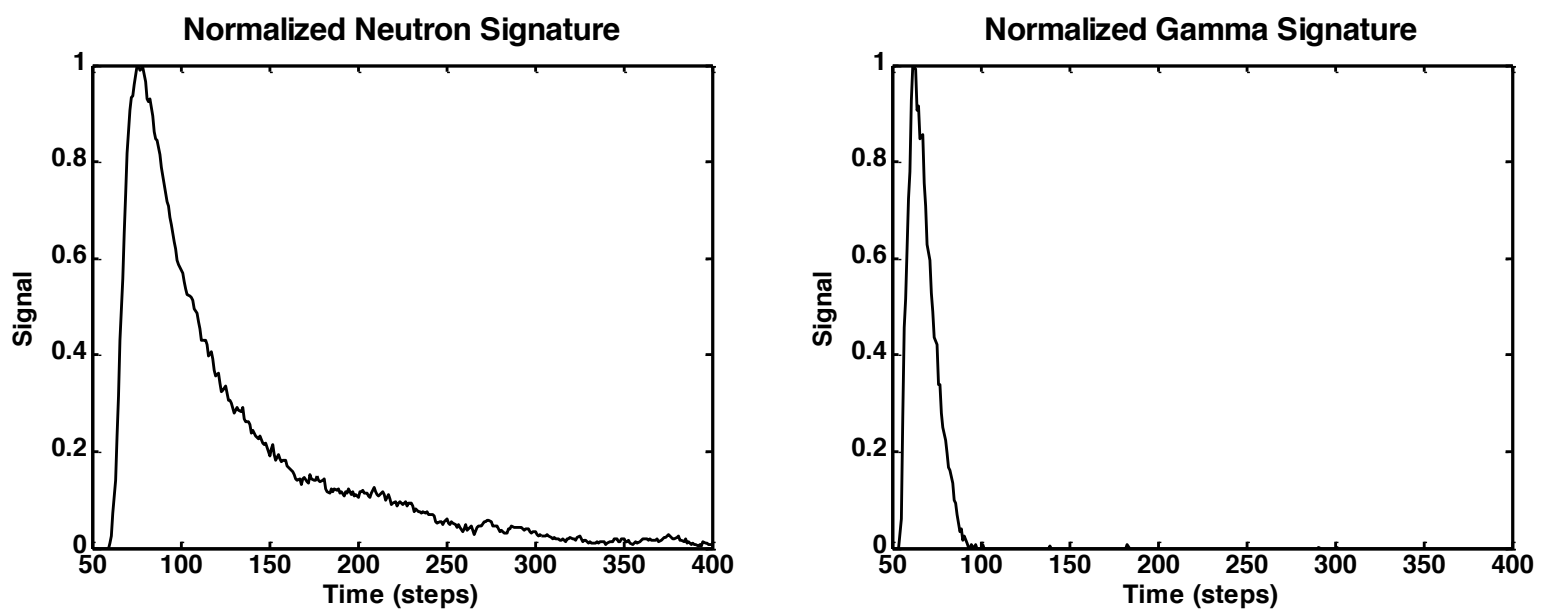

Figure 25. Neutron (left) and gamma-ray (right) signatures constructed from the sum of individual detections.

After the neutron and gamma ray signatures were defined, signal analysis of incoming data proceeded in a multi-stage process with the following steps.

A time window as short as possible $(<0.5 \mu \mathrm{s})$ was passed over the data to locate peaks, with an example shown in Figure 26. Several techniques were used to make a set of events. A simple threshold on peak height was used to describe event location in time. Additionally, only peaks that were the maximum value within the time window centered on them were accepted. All local maxima exceeding the threshold were held for further analysis. Event location (i.e., time) and amplitude of raw events were maintained for further analysis.

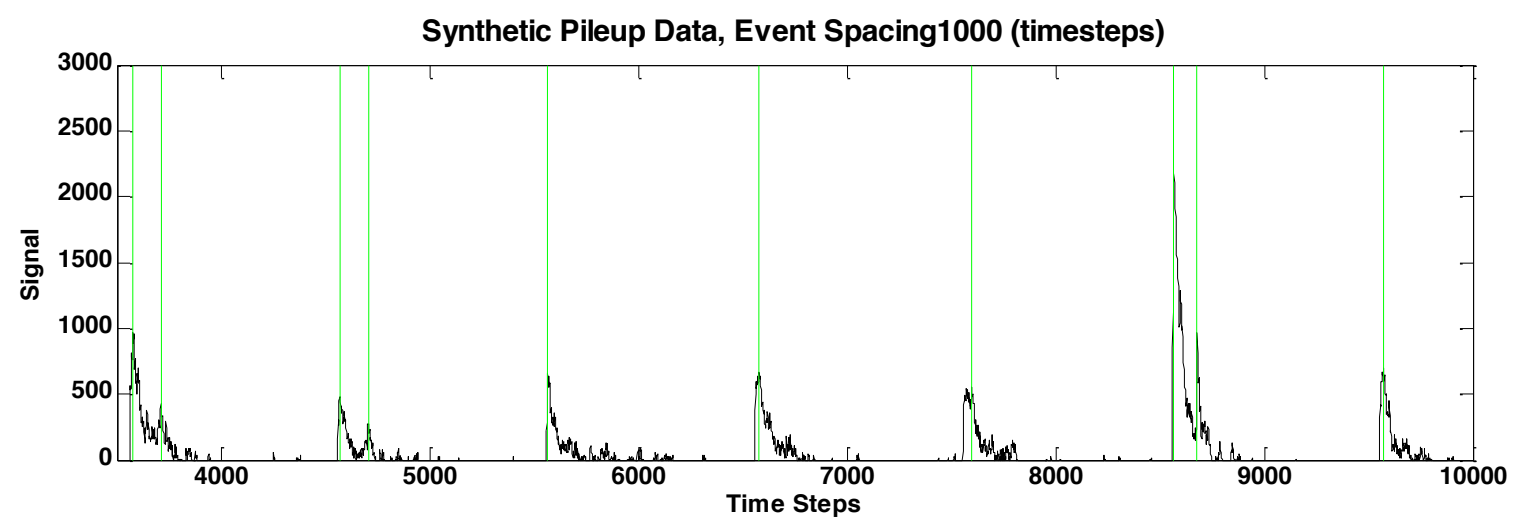

Figure 26. Example of detected event locations in time.

For each event located by the preceding step, a broad surrounding time window ( $7 \mu$ sor the test detector) was used to select any additional events; these were then grouped together. This broad window was chosen to group all events that may be piled-up with the currently analyzed event. These events are then fit as an ensemble to each permutation of neutron/gamma-ray signatures.

For each event, the most likely identification (i.e., neutron or gamma-ray) was made using a regression approach. In this approach, the permutations of possible event identification were made for each raw event in the group. In all cases, the resulting simulated data (containing neutrons or gamma- rays in the proper locations and event heights) are compared with the real measured data, and the overall sum of square errors used as a metric to determine the most 
likely ID for each event (Figure 27). In order to avoid overestimates of event size, the height of each peak was reduced to account for preceding overlapping events. Each event was again estimated in height relative to the preceding overlapping signals, and thrown out if it was not above a threshold. In this way the most likely identification for each event (neutron or gammaray) is maintained.

This approach is much faster than a "traditional" regression scheme, as the event location and height are not varied, but set by the preceding algorithm step. For this reason, this sort of regression can operate quickly in comparison with a scheme that relies on fitting of event number, location, and height as well as type.
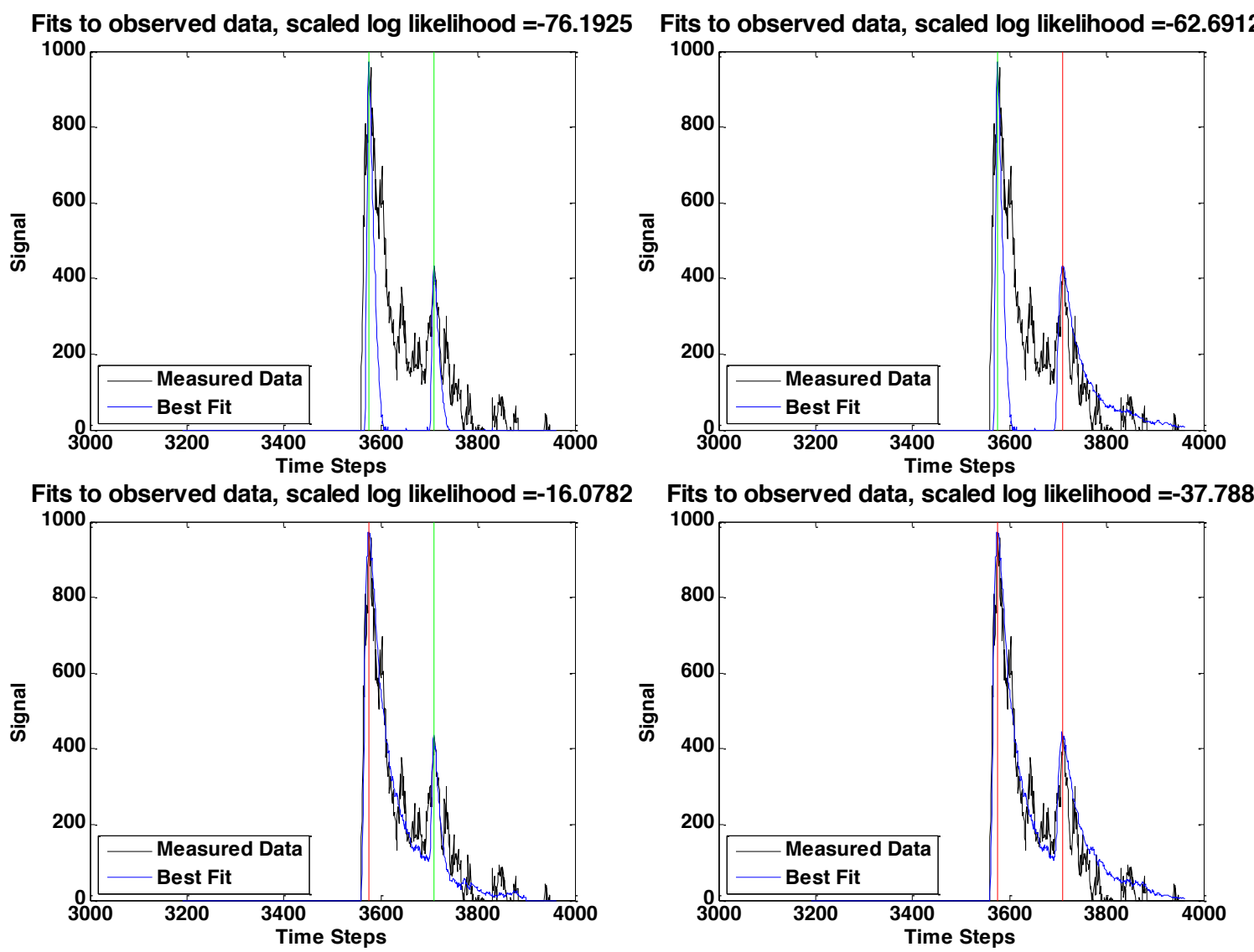

Figure 27. Simultaneous Evaluation of Multiple Events. Neutrons shown in red, gamma-rays in green. The most likely fit (single neutron, single gamma-ray) is shown in the bottom left image.

Using this multi-stage regression analysis technique, data can be characterized for arbitrarily sized time buffers, for data with or without pile-up. To characterize performance of this method for the test detector, "clean" gamma-ray and neutron signature sets were generated by manually inspecting data and constructing sets of each type of signal well separated from other confounding signals. Neutron-only and gamma- ray-only data were then sampled randomly to create desired synthetic data sets with and without pile-up, and with any desired ratio of neutron and gamma-ray events. Data sets with uniformly-spaced data were created and evaluated first (Figure 28). 


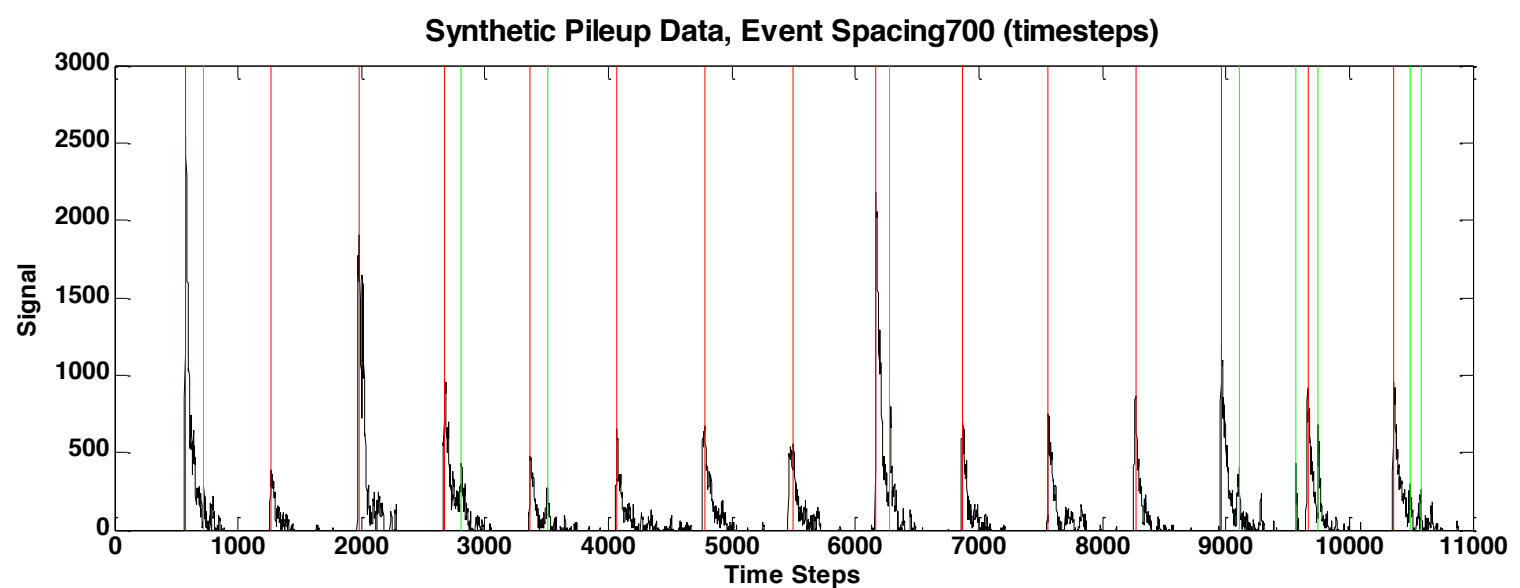

Figure 28. Synthetic pileup data, neutrons and gamma-rays.

Data with varying concentrations of neutrons and gamma rays were produced and tested to demonstrate the detection falloff for evenly spaced events (Figure 29). Performance of this technique is substantially enhanced relative to the earlier wavelet filter technique, providing good accurate neutron detection probability in the $\mathrm{MHz}$ range.

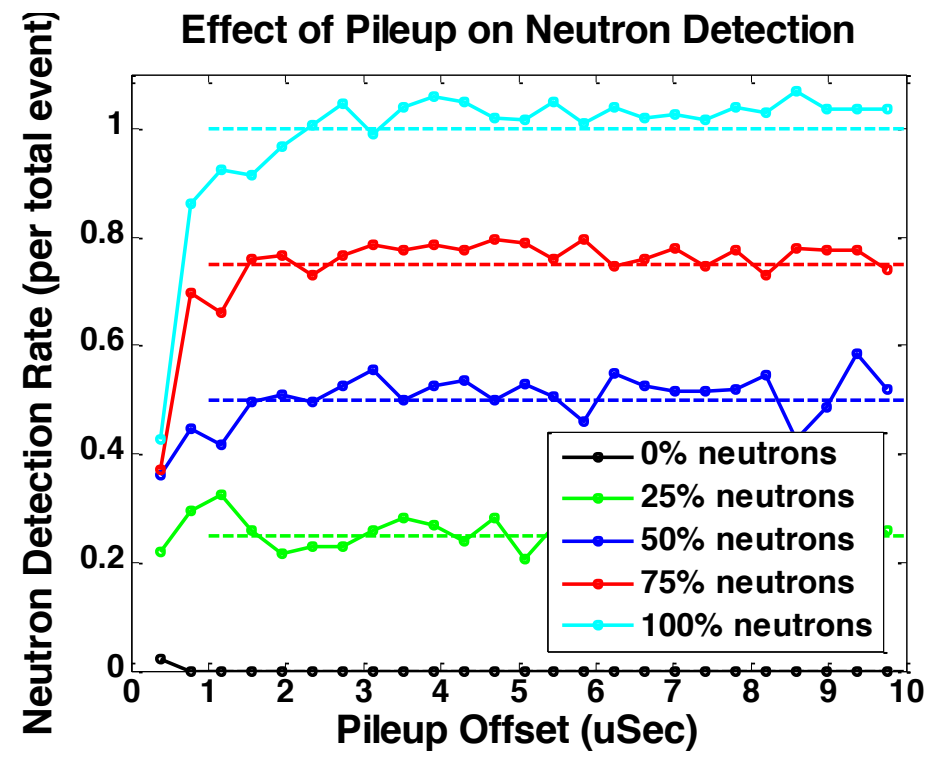

Figure 29. Performance of regression technique with uniformly spaced synthetic pileup data.

This exercise was repeated for data with randomly separated pulses to demonstrate the effect of realistic data on performance. In each case, a random standoff was picked to reproduce Poisson-distributed events; by definition, some events were closer, and some further away than average (Figure 30). Measured performance was slightly worse under these realistic standoff conditions (see Figure 31 as compared to Figure 29). While this softening and extending of performance falloff is likely due to the presence of realistic and intermittent very-high-pileup events, the overall detection rate remains relatively high in a range close to one $\mathrm{MHz}$. 
PNNL-23011

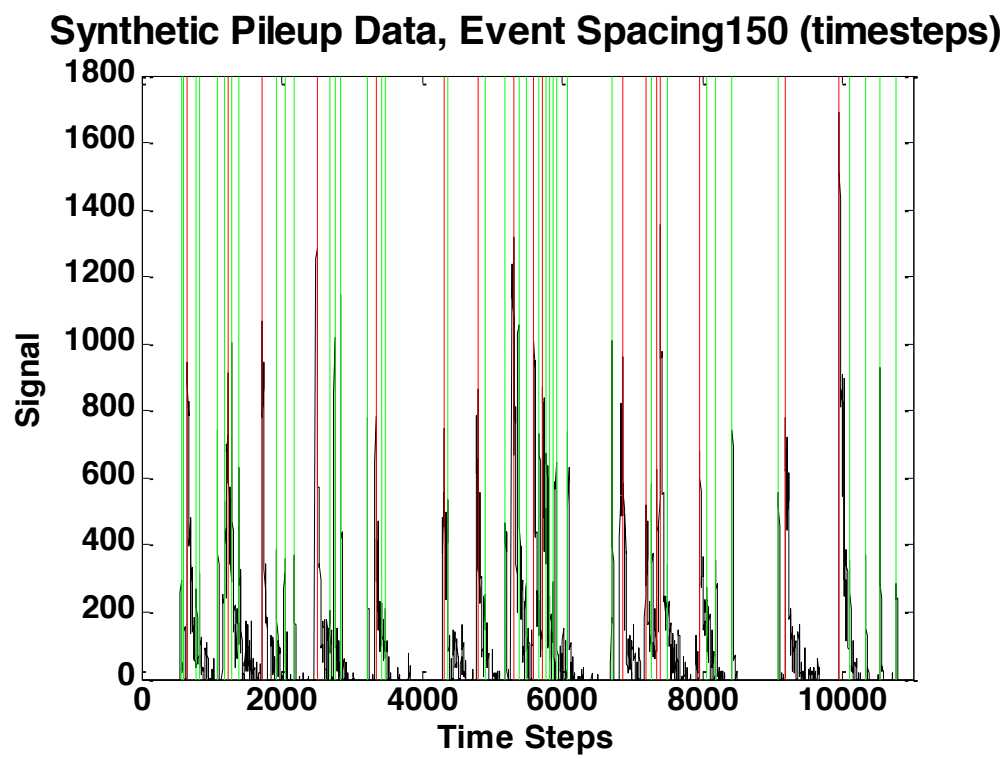

Figure 30. Randomly distributed synthetic pileup data.

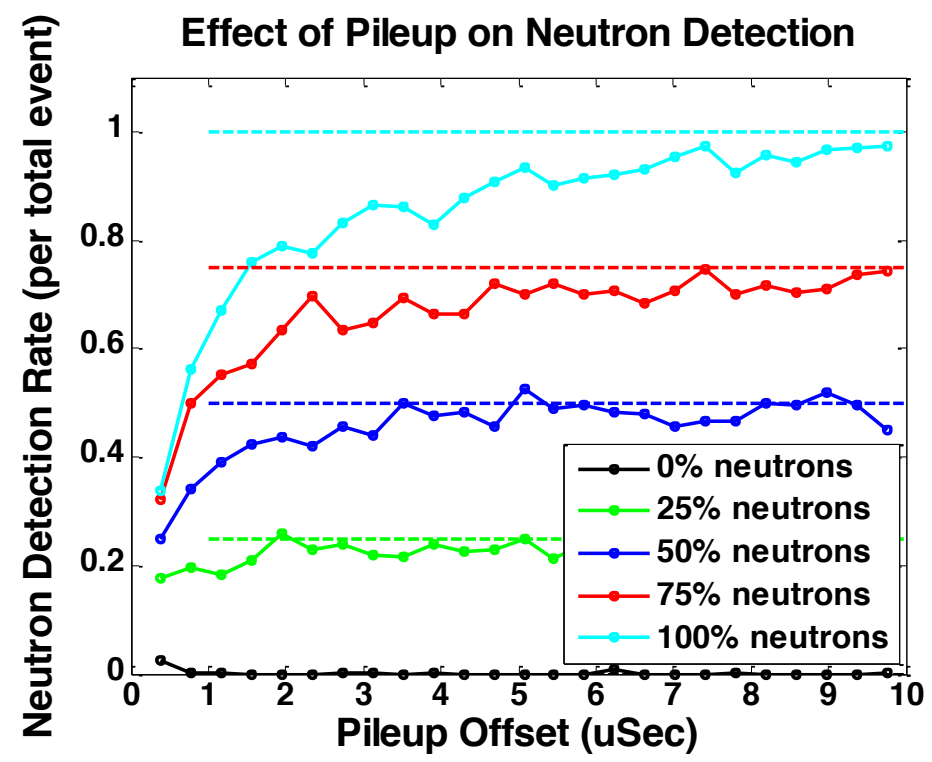

Figure 31. Performance of regression technique with randomly distributed synthetic pileup data.

A hybrid signal analysis technique, combining the pile-up pulse and gamma-ray discrimination, in which features in the raw data are compared with corresponding features in a filtered time series allows effective separation of neutron signatures with pile-up, up to substantial fractions of one $\mathrm{MHz}$ in total event rate. Future signal analysis work in the follow-on project will include implementation of these approaches on high-speed electronics and the combination of PSD and shift register based logic for multiplicity counting in real time. In particular, significant increase in the processing time in the regression technique will be required to achieve real time analysis. The present technique uses wide windows for regressions that include all adjacent peaks that may be relevant to the peak being investigated. As a result, this technique may fit and re-fit many closely spaced peaks, effectively requiring extraneous computation. The computation speed will be increased by considering the first peak in a wave train only once. This step is expected to amount to a significant computational time savings. This technique could then be adapted for fast electronics (e.g., field programmable gate array or FPGA technology). 


\section{Demonstrator System}

Based on the experiments with the bench-top system, a design of the demonstrator system was developed. The primary purpose of the demonstrator system was two-fold, first to provide a demonstration that the $\mathrm{LiF} / \mathrm{ZnS}$ material could be assembled into an operational detector, and to provide validation of the modeling and simulation results. The validation of the simulation results allows a more accurate estimate of the anticipated performance of the full-scale system, and supports the full-scale system design. Finally, the demonstration system allows exploration of the challenges and issues that might be associated with the full-scale system.

The demonstrator system was designed to be four paddles, each with the same active area as the bench-top, that is, $71.12-\mathrm{cm}$ (28in) active height by $15.24-\mathrm{cm}$ (6-in) active width. Four paddles were chosen to allow an experimental configuration surrounding a source and to provide a more realistic estimate of the efficiency and die-away times of the system.

As with the bench-top, the demonstrator paddles were comprised of five layers of LiF/ZnS material sandwiched between six layers of light guide. Due to the increased capability, the wavelength shifting light guides were used, and set at $0.7-\mathrm{cm}$ thickness. Although experiments indicate that $0.9-\mathrm{cm}$ light guides provide higher efficiency and perhaps better performance overall, the $0.7 \mathrm{~cm}$ was chosen as it provided the highest figure of merit simulation results. However, this thickness should be explored further in the full-scale system design.

The assembly of the paddles was straightforward; the LiF/ZnS sheets were simply placed on a WLSP light-guide. Another light guide was placed on top, and the process continued until there were five LiF/ZnS sheets sandwiched between six WLSP sheets. No material (glue) was used between the LiF/ZnS sheets, and the paddles held together in the final assembly by pressure. A non-scintillating PMMA light-guide was glued onto each end of the paddle. Initially, an optically clear Room Temperature Vulcanizing (RTV) silicone rubber was used, however, this did not provide a strong bond, and appeared to attenuate the light more than optical cement. The RTV was removed, and the light guides assembled with optical cement (St. Gobain $\mathrm{BC600)}$. The paddles and end light guides were wrapped with Teflon tape.

The assembled paddle with the end light-guides was then placed in aluminum box housing with external dimensions of $8.9-\mathrm{cm}$ (3.5-in) thick by $20.3-\mathrm{cm}$ (8-in) wide by $153.7-\mathrm{cm}(60.5$-in) high. Aluminum was chosen as a light-tight material that would provide minimal neutron attenuation, which is helpful to compare to modeling and simulation results. The top and bottom of the detector housing were assembled with rubber seals to ensure light was not able to enter the detector.

After the paddle was placed in the box, photomultiplier tubes (PMTs) were attached to the light guides on each end of the paddle. The PMTs were optically connected to the light guides using silicone optical grease (Eljen Technologies EJ-550) and held in place with rubber band tensioners. This allowed for some adjustment between the PMT and detector body to achieve the correct positioning within the aluminum housing.

The PMTs used were Hamamatsu R7724 two-inch PMTs. The voltage divider base was supplied by Ludlum Measurements, providing an integrated package. The PMTs operated with a positive bias at approximately $1700 \mathrm{~V}$. The PMTs were measured with a $\mathrm{Nal}(\mathrm{TI})$ crystal prior to assembly, and similar performing PMTs paired for each paddle. 
The high voltage and signal wiring were standard coaxial cables using either Safe High Voltage (SHV) for the high voltage or Bayonet Neill-Concelman (BNC) connectors for the signal, with internal wires routed inside the aluminum housing to a bulk-head connector on one end of the detector housing, which provided a light-tight seal. The paddles were then stood on end and arranged in a square as shown below in Figure 32. The size of the square was maintained by using a small aluminum bracket (shown at the bottom of the demonstrator in Figure 32) at the top and bottom and attached to the paddle housings. These demonstrator paddles are taller than would be required for a multiplicity counter, and must be moved to allow for the insertion of a source. For a full-scale system, the height of the paddles would need to be reduced in order to allow placement of the source in the counter without disassembly or use of a ladder.

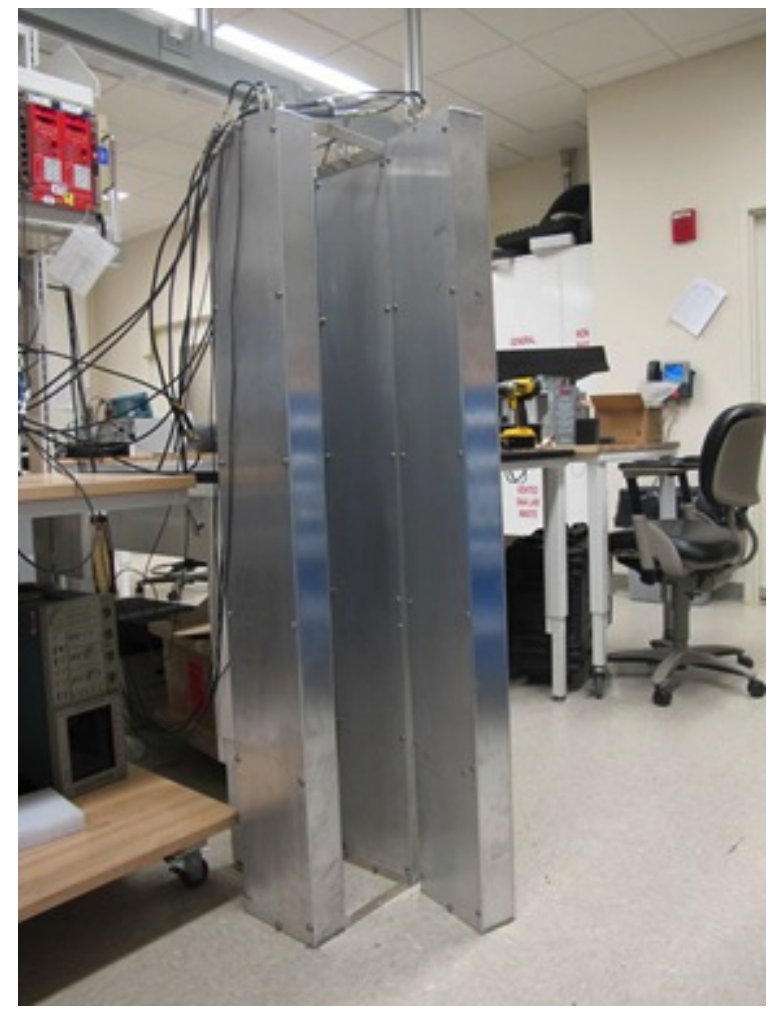

Figure 32. Demonstrator system of four paddles (three are visible) ready for insertion of the source for measurements.

The high voltage was supplied by two CAEN N470 power supplies that fit in a Nuclear Instrumentation Module (NIM) crate and can be seen in the upper left hand corner of Figure 32. Two modules were required to allow individual control of eight high voltage channels, corresponding to the eight PMTs.

The signal from the PMTs was connected directly to a digitization data collection system initially. No pre-amplifier was used, as the scintillation pulses out of the PMTs are large enough to be collected directly. The data was collected on a Digital Gamma Finder (DGF) Pixie-4 card manufactured by XIA (Hayward, CA). This card provides 14-bit analog to digital conversion (ADC) waveform acquisition at $75 \mathrm{MHz}$, which was sufficiently fast for this application. The bench-top used both the Pixie-500 and Pixie-4 and there did not appear to be significant differences between the two data acquisition speeds for this type of detector. The Pixie-4 is a card using the Compact PCI/PXI standard and was used in a PXI crate with an embedded controller (National Instruments). The standard XIA graphical user interface, the Pixie-Viewer, 
was used to collect the waveform data. Initially two cards were used, as each card can support up to four data channels. However, after some experiments and subsequent analysis, it was determined that requiring coincidence between the PMTs of each detector required the use of a dedicated card for each detector to avoid possible event loss. Due to the way the card processes data, there is a possibility of losing events during a coincidence event that is being written to the internal card memory. Although this may be accounted for properly in the live-time calculation, it is not a satisfactory method for use in multiplicity counters, where the fission neutrons arrive within a short time frame.

The collected waveform data was then analyzed in a post-processing mode. The data was first processed through the pulse shape discrimination (PSD) to separate the gamma-rays from the neutrons, producing an output file of neutron event times. These times were then input into a virtual shift register, which performed the same functionality as a shift register, providing the multiplicity distributions. The PSD algorithm was validated using data files collected using a gamma-ray-only source $\left({ }^{137} \mathrm{Cs}\right.$ ) as well as a neutron only source (heavily gamma-ray-shielded $\mathrm{AmBe}$ ), before being used on the neutron data collected for demonstration and comparison to simulation results $\left({ }^{252} \mathrm{Cf}\right)$. The virtual shift register was verified by collecting data in list mode from a ${ }^{3} \mathrm{He}$ based system, which was subsequently attached to a shift register. The list mode data was analyzed using the virtual shift register and compared with the shift register results, which was in fairly good agreement (3\% for singles, $20 \%$ for doubles and $7 \%$ for triples). The difference in the singles, doubles and triples calculated with the distributions from the traditional shift register and the list mode virtual shift register are due to the slightly different gate locations (the list mode shift register is "forward looking"). There is also dead-time present in the electronics used to produce the time-stamped pulses for use in the list mode shift register that affects the distributions. The discrepancy was not of concern for this analysis, but could be minimized with the use of electronics with less dead time, and by altering the gate locations in the virtual shift register.

During the data analysis of the die-away times, the results were significantly different than expected based on the modeling and simulation results. The expectation was that the die-away times should be fairly short, on the order of several tens of microseconds. However, the results from the analysis of the waveforms indicated die-away times larger than $100 \mu \mathrm{s}$. This inconsistency required some investigation as to the root cause, and ultimately was discovered to be significant dead time in the data acquisition of the digital waveform. The Pixie-4 system has a dead time associated with each processed event after it has been collected during the writing to the internal memory on the card itself. This dead time is $2 \mu$ s plus three times the waveform length. The waveform collection length was set at $3 \mu \mathrm{s}$, and therefore the dead time for the specific setup was $11 \mu \mathrm{s}$ per channel after each event. As this technology is expected to have die-away times on the order of $10 \mu \mathrm{s}$, a dead time of the same magnitude is not satisfactory. This issue is somewhat mitigated by the fact that there are four different modules in the demonstrator system, and the other three paddles would be ready to accept and process a signal during the dead-time on the first paddle. However, the analysis can become even more challenging, since after each event, the paddle or channel with the event would have this significant dead time. Accounting for, or making these dead-time corrections is non-trivial, and is an on-going issue even for the ${ }^{3} \mathrm{He}$ based systems, which have much less dead-time than this data acquisition system.

In order to demonstrate die-away times more consistent with the simulation results, a different data acquisition system was set up based on analog electronics. The pulses were amplified, input through a single channel analyzer, and then the two PMT signals from a single paddle were analyzed through a coincidence module, with the transistor-transistor logic (TLL) output 
fed to a JSR-14 shift register. This setup neglected any gamma-ray-neutron separation using PSD, and does have gamma-ray contamination as part of the results. In addition, to avoid pileup issues, a small neutron source was used, with a fairly low event rate. This type of data acquisition would therefore not be appropriate for a full-scale system for assay purposes, but did allow for data collection to produce an estimate of the die-away time that is much closer to model predictions. 


\section{Data Analysis and Comparison to Simulation Results}

For the demonstrator system, a number of measurements and experiments were carried out. However, since a primary purpose of the measurements was to provide better estimates of the capability of the full-scale system, this section will focus on the pertinent measurement results. The two measurements that were compared to the modeling and simulation results are the efficiency and die-away time.

A number of additional experiments and measurements were collected, but are not reported here as they were not the main focus of the demonstrator measurement campaign. Other measurements included measuring the response of individual PMTs to ensure the response of each was similar across the detectors. Measurements of single paddles or detectors were conducted on a regular basis to ensure similar response across the detectors during the measurement period. Measurements were conducted with the source in various locations in relationship to the center of the paddle to better understand the response across the active area of the detector.

The efficiency allows a better estimate of the Validation Correction Factor, which is needed to account for the losses that were not modeled. The model used a homogenous mixture of LiF, $\mathrm{ZnS}$, and binder that is not representative of the actual material. Therefore, the simulation was stopped at the neutron capture. The reaction products traveling to the $\mathrm{ZnS}$, the scintillation and light collection process, the PMT conversion to electronic signals and the data acquisition processes were not modeled, and losses from these were accounted for by the VCF. The VCF is a ratio of the measured neutron efficiency of the detector to the simulated neutron capture efficiency in the model. The data for the efficiency measurements was the digitized waveforms collected using the Pixie-4 data acquisition card. The data was post-processed through the simple dual window PSD algorithm to discriminate gamma-rays responses, and the net neutron rate normalized to the emission rate to determine the efficiency.

For the demonstrator system, the measurements were made with the paddles arranged around a central source opening as was shown in Figure 32. The efficiency data as stated previously was collected using the Pixie-4 data acquisition system with the digitized waveforms collected for post processing. Background and sample data were collected for $180 \mathrm{~s}$. Longer runs were challenging to achieve due to the current limitations of the data analysis routines (at this time implemented in MatLab), but were of adequate length to provide good demonstration data. The background was collected without any source present in the demonstrator system, but with the source holder (a cardboard stand) in place. Once the background data was collected, the ${ }^{252} \mathrm{Cf}$ source was placed in the center of the cavity, and in the center of the active area of the system, and measurements performed. The ${ }^{252} \mathrm{Cf}$ source on the measurement date (Nov 12, 2013) was $1.48 \mu \mathrm{Ci}$, corresponding to an emission rate of 6325 neutrons per second. The low activity source was used to minimize pile-up in the data stream, which complicates the data analysis and interpretation. The ${ }^{252} \mathrm{Cf}$ source was used in an unshielded (except for the stainless steel enclosure of the source itself) configuration, and a configuration surrounded by $5.08 \mathrm{~cm}$ (2 in) of lead to minimize gamma-ray contributions.

For the efficiency analysis the total rate of neutrons collected was used, and the analysis consisted of using pulse shape discrimination to determine the gamma-ray responses versus 
the neutron responses, and calculate a net neutron rate by subtracting off the neutron background rate from the sample rate. The rate calculation used the live-time of the system, resulting in a dead-time corrected rate. The efficiency (absolute) of the system is then just the detected neutron rate divided by the source emission rate.

The results of this data collection are provided in Table 7 . The first observation is that the gamma-ray contribution is a small percentage (1-2\%) of the total rate. This is largely due to the coincidence requirement between the PMTs, which significantly reduces the gamma-ray acceptance. Notice that the gamma-ray rate for both the sample and background does not depend on the configuration; adding lead did not reduce the accepted gamma-rays significantly. The net neutron rate was on the order of $400 \mathrm{cps}$, and therefore pile-up should not be very significant for the ZnS pulses. It is interesting that the lead shielded configuration resulted in a higher neutron count rate than the unshielded configuration, which was unexpected. However, the same behavior was produced in the simulation results, indicating a physics-based effect.

Table 7. Measured count rates with the demonstrator system. The uncertainty reported is statistical.

\begin{tabular}{|c|c|c|c|c|c|}
\hline Configuration & $\begin{array}{c}\text { Neutron } \\
\text { background } \\
\text { (cps) }\end{array}$ & $\begin{array}{c}\text { Gamma-ray } \\
\text { background } \\
\text { (cps) }\end{array}$ & $\begin{array}{c}\text { Neutron } \\
\text { sample (cps) }\end{array}$ & $\begin{array}{c}\text { Gamma-ray } \\
\text { sample (cps) }\end{array}$ & $\begin{array}{c}\text { Net neutron } \\
\text { rate (cps) }\end{array}$ \\
\hline $\begin{array}{c}\text { Unshielded } \\
{ }^{252} \mathbf{C f}\end{array}$ & $15.16 \pm 0.29$ & $7.99 \pm 0.21$ & $416.72 \pm 1.51$ & $13.99 \pm 0.28$ & $401.55 \pm 1.54$ \\
\hline $\begin{array}{c}\text { Pb-shielded } \\
{ }^{252} \mathbf{C f}\end{array}$ & $15.28 \pm 0.29$ & $8.33 \pm 0.21$ & $465.33 \pm 1.59$ & $13.90 \pm 0.28$ & $450.05 \pm 1.62$ \\
\hline
\end{tabular}

Once the efficiency data was collected and analyzed, the results could then be compared to simulation results from a model of the demonstrator system. The model included the 4 panels with aluminum housing, the source stand and the floor (Figure 33), the other room objects were assumed to be at an adequate distance to produce a negligible effect on the results. The simulation stops at the neutron capture, and therefore is not affected by electronics or gammaray misidentifications. The simulation provided neutron capture efficiency for the demonstrator system of $7.5 \%$ for the unshielded case and $8.6 \%$ for the lead-shielded case. Dividing the measured experimental efficiency by the modelled efficiency resulted in VCF of 0.84 and 0.83 respectively. These results are also provided below in Table 8.

Table 8. Measured and simulated efficiencies with the resulting VCF. The uncertainty in the measured efficiency (and hence VCF) is dominated by the uncertainty in the source activity.

\begin{tabular}{|l|c|c|c|}
\hline Configuration & $\begin{array}{c}\text { Measured Efficiency } \\
\mathbf{( \% )}\end{array}$ & $\begin{array}{c}\text { Simulated Efficiency } \\
(\mathbf{\%})\end{array}$ & VCF \\
\hline Unshielded $^{\mathbf{2 5 2}} \mathbf{C f}$ & $6.35 \pm 0.95$ & $7.50 \pm 0.08$ & $0.84 \pm 0.13$ \\
\hline Pb-shielded $^{\mathbf{2 5 2}} \mathbf{C f}$ & $7.12 \pm 1.06$ & $8.60 \pm 0.09$ & $0.83 \pm 0.12$ \\
\hline
\end{tabular}


PNNL-23011

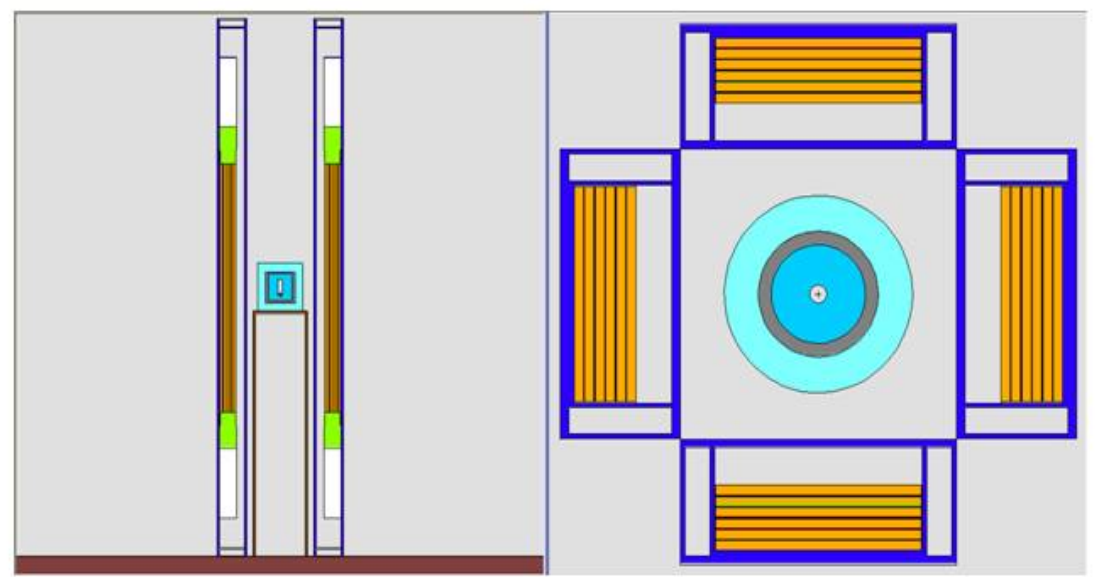

Figure 33. Model of the demonstrator system, shown here with the source holder and a moderated source.

The die-away time is a measure of the residence time of the neutrons in the system after the first neutron in a fission event has been detected. It is a parameter of the system that is driven primarily by the moderation of the neutrons, and is therefore not significantly affected by the losses in the detector after neutron capture. The simulation results for the die-away time should be in good agreement with the experimental data, and the die-away time comparison is a method to validate the modeling and simulation.

The die-away time data was collected using analog electronics to analyze for coincidence pulses between the two PMTs on a single detector, and then generate TTL pulses, which were subsequently fed into a JRS-14 shift register. This method of data collection was employed due to the significant dead time associated with the read-out of the waveform when using the digitizer data acquisition system. The dead time did not have a significant effect on the efficiency measurements since the event rate was low, and only measuring the single or total neutron events, not measuring multiplicities, as is required for the die-away time measurements. The waveform acquisition could have affected the efficiency results to a small extent, but would decrease the efficiency, and therefore, the efficiency estimation is conservative, that is, the performance may increase with better data acquisition methods. The analog method was not used for the efficiency measurements, as the analog method did not employ pulse shape discrimination, and the gamma-ray responses were included in the data stream, although demonstrated above to be a small fraction of the overall rate.

To demonstrate that the gamma rays had little influence on the die-away measurements, the source was used bare (except for the stainless steel encapsulation) and surrounded by two inches of lead. The two inches of lead reduced the gamma-ray emission from the ${ }^{252} \mathrm{Cf}$ source by a factor of 6.9 as measured (separately) with a $\mathrm{Nal}(\mathrm{TI})$ gamma-ray detector that was $5.08 \times 10.16 \times 40.64 \mathrm{~cm}^{3}\left(2 \times 4 \times 16 \mathrm{in}^{3}\right)$. The two measurements show little change in the dieaway time, demonstrating the gamma rays had little influence in these measurements.

For these measurements, the pre-delay on the shift register was set to $4 \mu \mathrm{s}$, and the delay between the triggered neutron gate and the accidental gate at $4.5 \mathrm{msec}$. Initially, the background rate for singles and doubles was measured, and subsequently subtracted from the sample measurements to produce net rates. Measurements with various gate widths were made, both without and with the lead shielding in place. Each measurement was taken for five 
minutes. The resulting rates are shown below in Table 9. For the singles rates, the statistical uncertainty for the five minute run was less than $1 \%$, corresponding to a few counts per second, and appears to be consistent within the statistical uncertainty across the data collections with the various gate widths as expected. There is a significant increase in the singles rates (13\%) when the lead shielding is introduced, which was also observed with the digital electronics during the efficiency measurement. The simulated neutron capture efficiency with the lead shielding included increased by $15 \%$ over the neutron capture efficiency with the bare source. The similarity in response between the measured and simulated rates with and without lead shielding suggests that the increase in the count rate is due to the lead shielding affecting the neutron spectrum. The doubles rates are different between the two shielding configurations as well, with increased doubles for the shielded case, consistent with the increased singles rates. The doubles rate increases and then appears to reach a relatively constant value when using gate widths larger than about $40 \mu \mathrm{s}$. The doubles rates are not a smooth function (more easily observed in the plot of Figure 34 below), which may indicate a distribution containing several exponential distributions. This may be better characterized by a Rossi-alpha type plot of the data, but was not produced in this analysis.

Table 9. Single and doubles measurements used to estimate the die-away time. The uncertainties are statistical only.

\begin{tabular}{|c|c|c|c|c|}
\hline \multirow{2}{*}{ Gate Width $(\boldsymbol{\mu s})$} & \multicolumn{2}{|c|}{ Bare } & \multicolumn{2}{c|}{ Lead Shielded ${ }^{\mathbf{2 5 2}} \mathbf{C f}$} \\
\cline { 2 - 5 } & Singles Rate $(\mathrm{cps})$ & Doubles Rate $(\mathrm{cps})$ & Singles Rate $(\mathrm{cps})$ & Doubles Rate (cps) \\
\hline 8 & $382.43 \pm 1.13$ & $9.80 \pm 0.18$ & $430.23 \pm 1.20$ & $12.70 \pm 0.21$ \\
\hline 16 & $382.63 \pm 1.13$ & $14.53 \pm 0.22$ & $430.44 \pm 1.20$ & $18.47 \pm 0.25$ \\
\hline 24 & $382.61 \pm 1.13$ & $16.78 \pm 0.24$ & $430.21 \pm 1.20$ & $21.86 \pm 0.27$ \\
\hline 32 & $382.69 \pm 1.13$ & $18.20 \pm 0.25$ & $430.36 \pm 1.20$ & $22.88 \pm 0.28$ \\
\hline 40 & $383.78 \pm 1.13$ & $19.23 \pm 0.25$ & $430.77 \pm 1.20$ & $24.39 \pm 0.29$ \\
\hline 48 & $383.17 \pm 1.13$ & $21.29 \pm 0.27$ & $431.87 \pm 1.20$ & $26.98 \pm 0.29$ \\
\hline 56 & $384.11 \pm 1.13$ & $21.03 \pm 0.26$ & $430.06 \pm 1.20$ & $27.31 \pm 0.30$ \\
\hline 64 & $385.22 \pm 1.13$ & $21.54 \pm 0.27$ & $431.45 \pm 1.20$ & $26.49 \pm 0.30$ \\
\hline
\end{tabular}

The doubles rates were plotted (shown in Figure 34 below) and an exponential function was minimized to determine the die-away time according to the equation:

$$
D=A \cdot \exp (-G / \tau)
$$

where $D$ is the doubles rate, $A$ is a constant, $G$ is the gate width, and $t$ is the die-away time. 
PNNL-23011

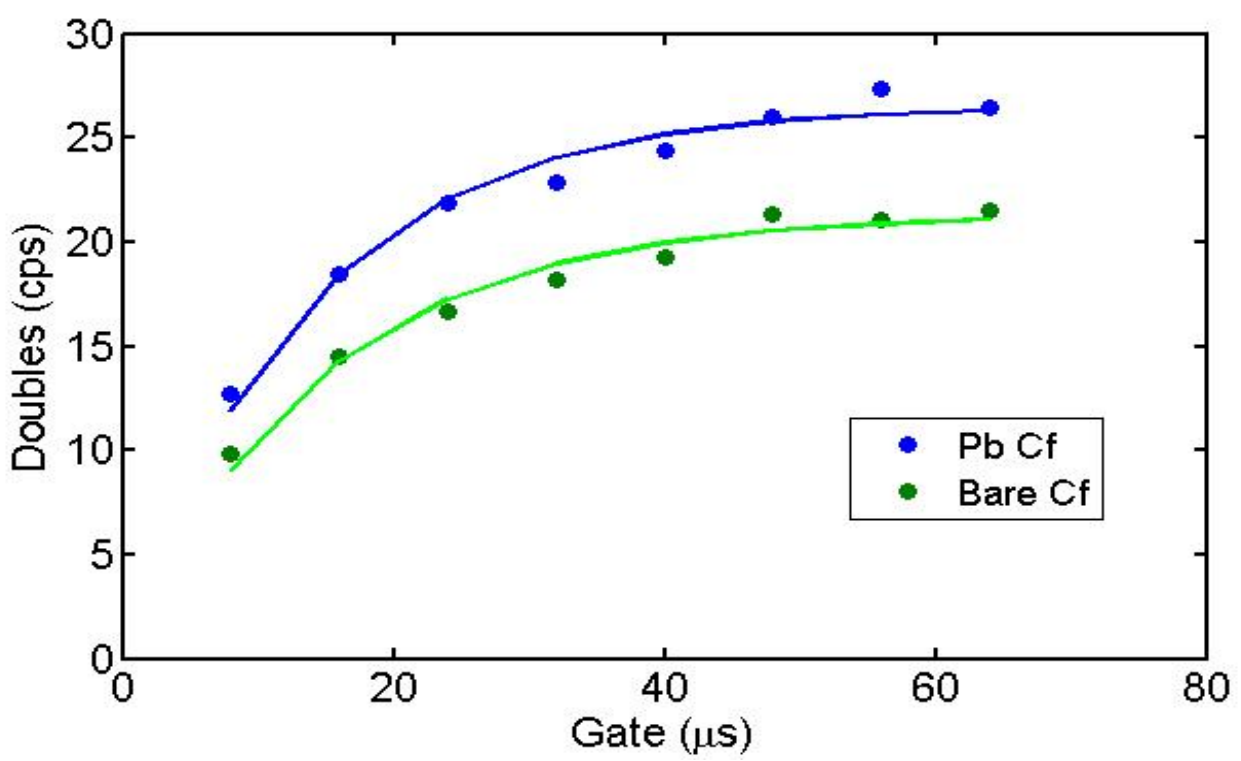

Figure 34. Doubles rate as a function of the gate width for the bare and lead $(\mathrm{Pb})$ shielded ${ }^{252} \mathrm{Cf}$ source.

The measurement (and simulation) result values are shown below in Table 10; these indicate that adding two inches of lead around the ${ }^{252} \mathrm{Cf}$ source does not change the die-away time significantly. Since the die-away time does not change dramatically, the gamma-ray responses in this data stream (due to the lack of PSD in this analog data acquisition) do not appear to modify the die-away time significantly. Therefore, these results should be consistent to what would be obtained with PSD, and can be compared directly to the simulation results (which only contain neutron captures).

The die-away time for these measurements was approximately $14 \mu \mathrm{s}$; a little higher for the unshielded source, a little lower for the shielded configuration. This is compared to the simulation results using a model of the demonstrator system, which provided a die-away time of approximately $12 \mu \mathrm{s}$. The uncertainties in these fits to data were not calculated or reported here. The comparison between the measurement and simulation results is in good agreement considering the possible gamma-ray responses in the data stream, the dead-time issue of the electronics, and the fact that the simulation stopped at the neutron capture. This good agreement provides confidence that the simulation provides values in the same range as measurements, resulting in a more confident estimate for the full-scale system.

Table 10. Die-away time estimates from measurements for the demonstrator system.

\begin{tabular}{|l|l|l|l|l|}
\cline { 2 - 5 } \multicolumn{2}{c|}{} & \multicolumn{2}{l|}{ Bare ${ }^{\mathbf{2 5 2}} \mathbf{C f}$} & \multicolumn{2}{l|}{ Lead Shielded ${ }^{\mathbf{2 5 2}} \mathbf{C f}$} \\
\cline { 2 - 5 } \multicolumn{1}{c|}{} & Measured & Simulated & Measured & Simulated \\
\hline $\begin{array}{l}\text { Die-away time }(\tau) \\
{[\mu \mathrm{s}]}\end{array}$ & 14.46 & 11.84 & 13.49 & 11.79 \\
\hline
\end{tabular}




\section{Summary and Conclusions}

Three different alternative technologies have been investigated for use in development of a ${ }^{3} \mathrm{He}$ free replacement for the Epithermal Neutron Multiplicity Counter. These include $\mathrm{BF}_{3}$ proportional tubes, boron-lined proportional tubes, and LiF/ZnS with wavelength shifting light guides. Models of each technology were developed and simulations performed and numerous optimization studies conducted. The ENMC model using ${ }^{3} \mathrm{He}$ was used as a baseline for comparison, and models developed and validated against this standard. For each technology, the studies started with implementing the new technology into a very similar configuration as the ENMC, and then investigating possible optimization approaches. Ultimately, a bounding configuration was investigated for each technology, which determined the highest figure of merit achievable while maintaining a reasonable footprint (not significantly larger than the ENMC).

The bounding models that maximized the figures of merit are provided in Figure 35 for the three alternative and the ENMC simulation results. Overlaid on the data are constant FoM2 (left hand plot) and FoM3 (right hand plot) values providing references for comparison. The boron-lined straw tube bounding model with 4725 tubes has fairly low efficiency and also larger die-away times compared to the ENMC. The $\mathrm{BF}_{3}$ bounding case with 99 tubes has increased efficiency compared to the boron-lined tubes, but also larger die-away times, resulting in figure of merit values 1.5-2.5 larger than the boron-lined bounding case, but still a factor of 2-3 lower than the ENMC. The LiF/ZnS bounding case of 20 layers of trapezoidal paddles also has lower simulated efficiency compared to the ENMC, but a short die away time, resulting in a FoM2 value that exceeds the ENMC, and a FoM3 that is slightly lower.
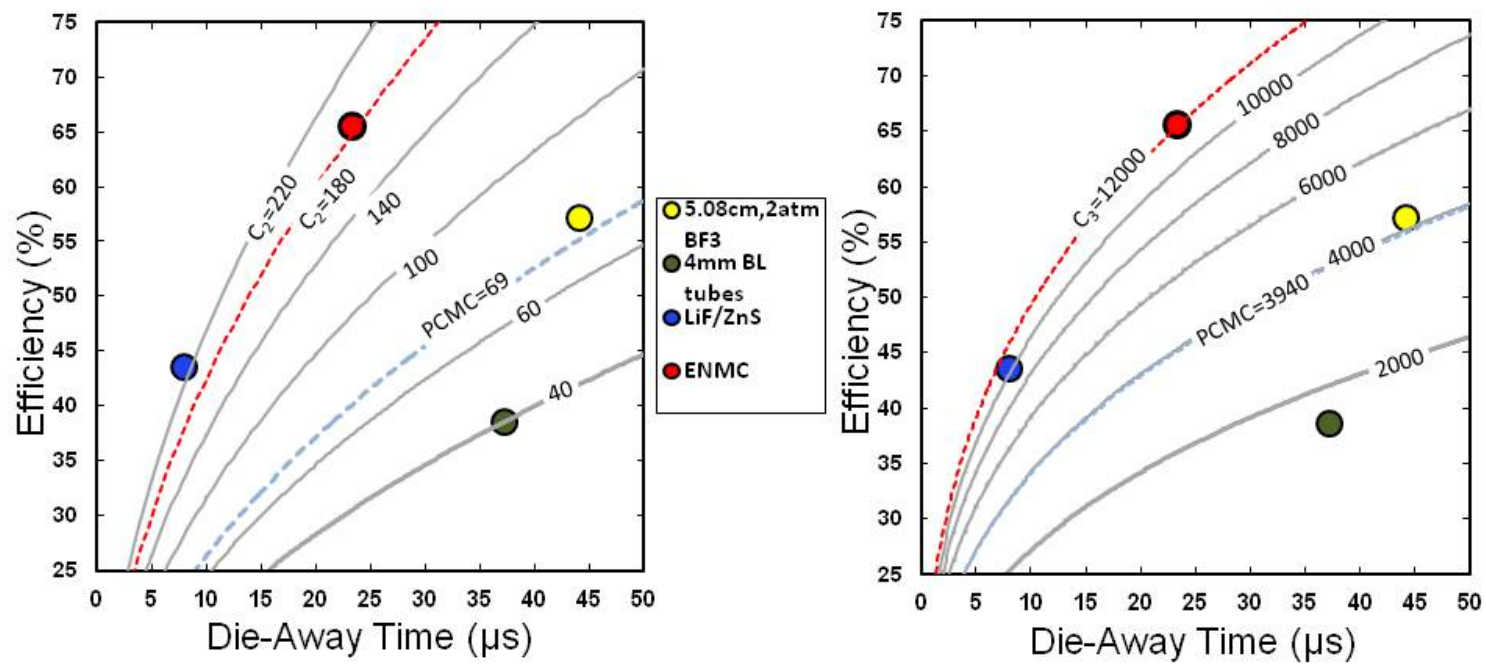

Figure 35. Summary of the optimized modeling results of the three alternatives along with the ${ }^{3} \mathrm{He}$ baseline. The constant FoM curves are overlaid on the data: FoM2 of the left and FoM3 on the right along with the ENMC values (dashed lines).

Based on the simulation results, the LiF/ZnS alternative was selected as the alternative technology to move forward with for the demonstrator system. Materials were purchased for a single detector paddle to build a bench-top system, and experiments performed to optimize and finalize the demonstrator design. The demonstrator consists of four paddles, each $71.12-\mathrm{cm}$ (28-in) active height by $15.24-\mathrm{cm}(6-\mathrm{in})$ wide by approximately $5.08-\mathrm{cm}$ (2-in) deep. Each paddle has a light guide on each end connected to a PMT, and housed in an aluminum case, for 
overall external dimensions of $8.9-\mathrm{cm}$ (3.5in) thick by $20.3-\mathrm{cm}$ (8-in) wide by $153.7-\mathrm{cm}$ (60.5-in) high. The active height is the same as the ENMC.

Analysis algorithms were explored to address pulse shape discrimination for reducing the gamma-ray misidentification as neutrons, and the pulse pile-up that will be an issue for high rate measurements. Promising results have been obtained using a hybrid algorithm that addresses both the gamma-ray discrimination and pile-up issues using a multi-stage regression analysis technique.

The effect of gamma-ray misidentification on the mass estimate was investigated using a theoretical approach. From this study, it appears that the LiF/ZnS material could provide good mass estimates even with the poorer gamma-ray discrimination capabilities compared to ${ }^{3} \mathrm{He}$ based detectors. Further work is needed to verify this result, but combined with the pulse shape discrimination work it indicates this alternative technology could be a viable replacement for ${ }^{3} \mathrm{He}$ in the ENMC.

Measurements were made with the demonstrator system, and models developed of the system to perform simulations. The simulations were required to provide a better estimate of the VCF needed to estimate the neutron detection efficiency of the full-scale system (as opposed to the neutron capture efficiency). Simulations of the die-away time were also compared to measurement, to help support the validation of the simulation results, and provide more confidence in the estimated capability of the full-scale system.

The measured absolute detection efficiency for a point source of unshielded ${ }^{252} \mathrm{Cf}$, centered in the source cavity of the demonstrator system was $6-7 \%$ using pulse shape discrimination with an estimated gamma-ray discrimination ${ }^{2}$ factor of $3 \times 10^{-8}$. This is compared to the simulated neutron capture efficiency of $7.5 \%$. The ratio of these efficiencies is 0.84 , which is the VFC. Using this VCF for the full-scale simulated system, the LiF/ZnS alternative appears to be a viable replacement for ${ }^{3} \mathrm{He}$ for the ENMC, providing the same capability in terms of figures of merit.

A die-away time for the demonstrator system was estimated from measurements to be $\sim 14 \mu \mathrm{s}$, and compared well with the simulation results of $12 \mu \mathrm{s}$. This consistency provides additional confidence in the simulation results for the full-scale system.

The full-scale system (design was shown in Figure 3) simulations were updated using the VCF obtained from the demonstrator system. The estimated efficiency of this design from simulation results is $63 \%$ compared to the ENMC at $66 \%$, while the die-away time is estimated at approximately $10 \mu \mathrm{s}$ compared to the ENMC die-away time of $23 \mu \mathrm{s}$. The LiF/ZnS alternative design is expected to exceed the performance of the ENMC in terms of the FoM2 values. However, this simulated performance may not appropriately include all the factors that would be associated with an actual full-scale LiF/ZnS based system.

Based on the modeling and simulation results and demonstration measurements made during the course of this project, it appears that LiF/ZnS is the only near-term viable alternative technology that can meet or exceed the capability of the ${ }^{3} \mathrm{He}$ based ENMC. Although this

\footnotetext{
${ }^{2}$ Gamma-ray discrimination is a measure of the ability of a neutron detector not to classify a gamma ray induced signal as a neutron, and is measured as the number of gamma rays identified as neutrons divided by the number of gamma rays incident on the detector.
} 
project has demonstrated the promising capability of the LiF/ZnS alternative, there are issues to explore and solutions to be developed for this alternative to be fully realized in an operational system. The major challenges based on the experience of this project are the pulse pile-up issue due to the long luminescence lifetime of the $\mathrm{ZnS}$, the gamma-ray sensitivity of the ZnS requiring discrimination, and engineering challenges to design a system with a size comparable to the ENMC. Other possible issues also need exploring include source height dependency, source energy dependence, and stability against temperature fluctuations during measurements. In order to fully investigate these possible challenges, a full-scale system needs to be designed and built, with solutions implemented, to provide a platform for testing and evaluation. 


\section{Appendix A: References}

1. Kouzes, RT. 2009. "The ${ }^{3} \mathrm{He}$ Supply Problem." Pacific Northwest National Laboratory Report PNNL-18388.

2. Pickrell MM, K Veal, and N Ensslin. 2007. "Fast and Epithermal Neutron Multiplicity Counters." Chapter 8 of Passive Nondestructive Assay of Nuclear Materials 2007 Addendum, Los Alamos National Laboratory Report LA-UR-07-1602.

3. X-5 Monte Carlo Team, MCNP-A General Monte Carlo N-Particle Transport Code, Version 5 - Vol. I: Overview and Theory, 2003, revised 2008, Los Alamos National Laboratory Report LA-UR-03-1987.

4. Van Ginhoven, RM, RT Kouzes, and DL Stephens. 2009. "Alternative Neutron Detector Technologies for Homeland Security," Report PNNL-18471, Pacific Northwest National Laboratory, Richland, WA.

5. Kouzes, RT, JH Ely, LE Erikson, WJ Kernan, AT Lintereur, ER Siciliano, DL Stephens, DC Stromswold, RM Van Ginhoven, and ML Woodring. 2010. "Neutron Detection Alternatives for Homeland Security." Nuclear Instruments and Methods in Physics Research A 623, pp 1035-1045.

6. Reilly D, N Ensslin, H Smith, Jr., and S Kreiner, Passive Nondestructive Assay of Nuclear Materials, United States Nuclear Regulatory Commission, 1991, ch. 16.

7. Ely, JH, ER Siciliano, MT Swinhoe, and AT Lintereur. 2009. "Modeling and Simulation Optimization and Feasibility Studies for the Neutron Detection without Helium-3 Project" Pacific Northwest National Laboratory Report PNNL-22228.

8. Swinhoe, MT, JS Hendricks, and DR Mayo. 2004. "MCNPX for Neutron Multiplicity Detector Simulation," Los Alamos National Laboratory Report LA-UR-04-8025.

9. MCNP6 - 1, May 2013 Monte Carlo Codes (XCP-3), X Computational Physics Division System Design \& Analysis (NEN-5), Nuclear Engineering \& Nonproliferation Division Los Alamos National Laboratory, mcnp6@lanl.gov

10. Veenhof, R. Garfield. http://garfield.web.cern.ch/ 2010.

11. Ely JH, ER Siciliano, and MT Swinhoe. 2012. "Alternatives to Helium-3 for Neutron Multiplicity Detectors." In Proceedings of the 52nd Annual Meeting of the Institute of Nuclear Materials Management.

12. Lintereur AT, JH Ely, JL Rogers, RT Kouzes, and ER Siciliano. 2012. "Boron-10 Lined Proportional Counter Model Validation." Pacific Northwest National Laboratory Report PNNL-SA-92071.

13. Chung K, MT Swinhoe, M lliev, and KD lanakiev. 2013. "Measurements and Simulation of a Boron-10 Lined Proportional Counter for MCNPX Benchmarking." Los Alamos National Laboratory Report LA-UR-13-27137.

14. Kouzes RT, JH Ely, LE Erikson, WJ Kernan, DC Stromswold, and ML Woodring. 2010. "Full Scale Coated Fiber Neutron Detector Measurements." Pacific Northwest National Laboratory Report PNNL-19264.

15. Ensslin N, WC Harker, MS Krick, DG Langner, MM Pickrell and JE Stewart. 1998. "Application Guide to Neutron Multiplicity Counting." Los Alamos National Laboratory Report LA-13422-M. 
16. Pazsit I, and SA Pozzi. 2005. "Calculation of Gamma Multiplicities in a Multiplying Sample for the Assay of Nuclear Materials." Nuclear Instruments and Methods in Physics Research A 555340.

17. Oberer, RB. 2002. "Fission Multiplicity Detection with Temporal Gamma-Neutron Discrimination from Higher Order Time Correlation Statistics." Ph.D. Thesis, Georgia Institute of Technology.

18. Donoho, D.L. 1993. "Nonlinear Wavelet Methods for Recovery of Signals, Densities, and Spectra from Indirect and Noisy Data". Symposia in Applied Mathematics. San Antonio, TX: American Mathematical Society.

19. Freeman, P.E., et al. 2002. "A Wavelet-Based Algorithm for the Spatial Analysis of Poisson Data”. Astrophysical Journal Supplement Series 138(1) 185. 


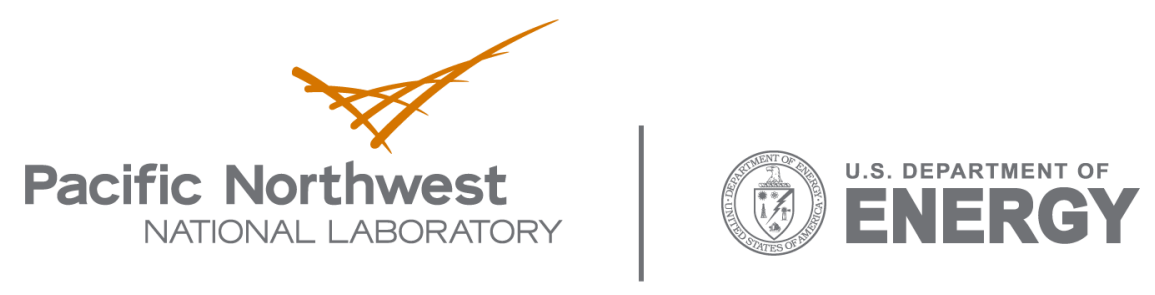

902 Battelle Boulevard

P.O. Box 999

Richland, WA 99352

1-888-375-PNNL (7665)

www.pnl.gov 